Supporting Information

\title{
Optimization of a simulated moving bed unit within an existing and revamped aromatics complex with crystallization and toluene methylation units
}

Jonathan C. Gonçalves*, Rui P. V. Faria, Alexandre F. P. Ferreira, Alírio E. Rodrigues

Correspondence: jcgs@fe.up.pt, Laboratory of Separation and Reaction Engineering Laboratory of Catalysis and Materials (LSRE-LCM), Department of Chemical Engineering, University of Porto, Rua Dr. Roberto Frias, s/n, 4200-465 Porto, Portugal.

\section{Table of Contents}

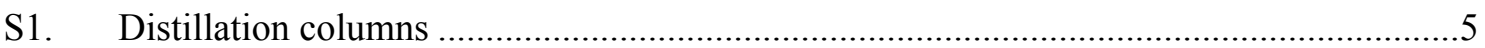

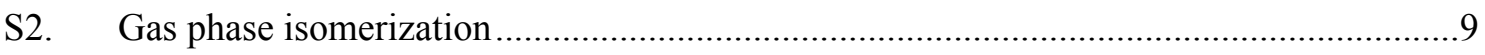

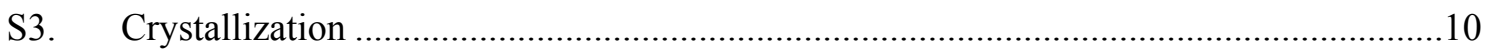

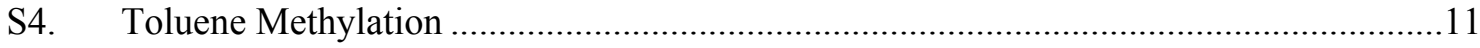

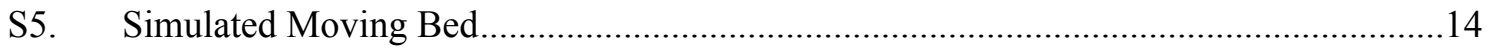

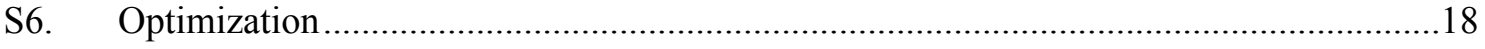

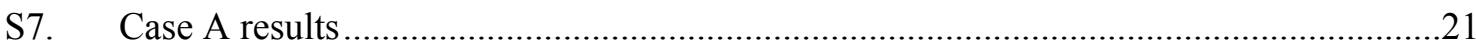

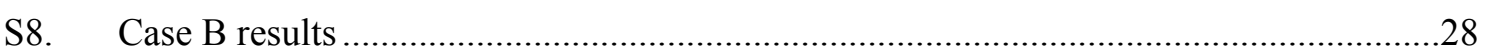

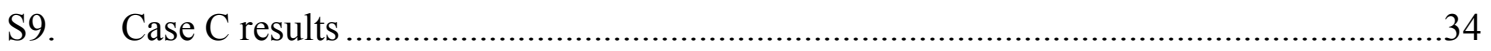

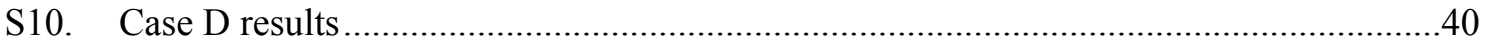

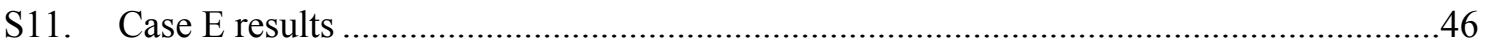

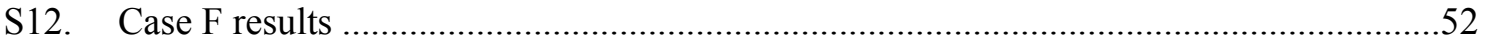

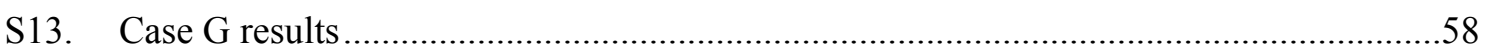

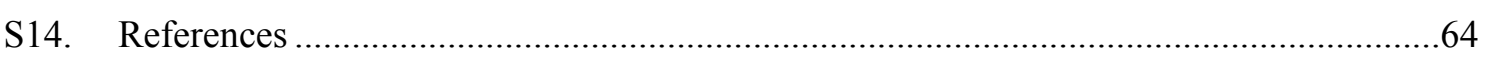




\section{List of Figures}

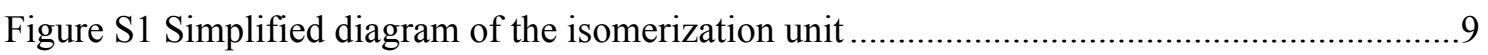

Figure S2 Temperature profile for the Feed/Effluent Exchanger for the base case A0 ..............10

Figure S3 Simplified diagram of the toluene methylation unit ...............................................11

Figure S4 Temperature profile of the Feed/Effluent Exchanger after step 5 of the optimization of the modified aromatics complex with toluene methylation and crystallization at $218 \mathrm{~K}$ (E5) ....12

Figure S5 Temperature profile of the Feed/Effluent Exchanger after step 5 of the optimization of the modified aromatics complex with toluene methylation and crystallization at $244 \mathrm{~K}$ (F5)....13

Figure S6 Internal concentration profile of the SMB unit for the case A after step 4 (A4): A) calculated as SMB at the middle of the switching time after the cycle steady state was reached and B) calculated using the TMB approach

Figure S7 Desorbent ( $p$-diethylbenzene - PDEB) in the extract and raffinate for fixed feed (62.3 $\left.\mathrm{m}^{3} / \mathrm{h}\right)$ and extract purity $(99.82 \mathrm{wt} \%)$ and several recovery ratios

Figure S8 Desorbent ( $p$-diethylbenzene - PDEB) in the extract and raffinate and p-xylene (PX) in the extract for fixed feed $\left(80.3 \mathrm{~m}^{3} / \mathrm{h}\right)$ and recovery $(99.987 \%)$ and several recovery ratios. .17

\section{List of Tables}

Table S1 Simulation of the benzene column in the base case (A0) using rigorous model RadFrac from commercial software Aspen

Table S2 Simulation of the splitter in the base case (A0) using rigorous model RadFrac from commercial software Aspen

Table S3 Simulation of the extract column in the base case (A0) using rigorous model RadFrac from commercial software Aspen

Table S4 Simulation of the raffinate column in the base case (A0) using rigorous model RadFrac from commercial software Aspen.....

Table S5 Simulation of the $o$-xylene column in the base case (A0) using rigorous model

RadFrac from commercial software Aspen.

Table S6 Utility consumption of an Isomar unit with a capacity of 40000 BPD Parex raffinate 10

Table S7 Utility consumption of a Crystallization unit with a capacity of 350000 ton/year $p$-xylene ${ }^{\mathrm{a}}$.

Table S8 Kinetic parameters for toluene methylation from Valverde ${ }^{3}$

Table S9 Mass balance for the toluene methylation unit after step 5 of the optimization of the modified aromatics complex with toluene methylation and crystallization at $218 \mathrm{~K}$ (E5)..........12

Table S10 Mass balance for the toluene methylation unit after step 5 of the optimization of the modified aromatics complex with toluene methylation and crystallization at $244 \mathrm{~K}$ (F5) ..........13

Table S11 Simulation of the distillation column within the toluene methylation unit after step 5 (E5 and F5) using rigorous model RadFrac from commercial software Aspen..........................14

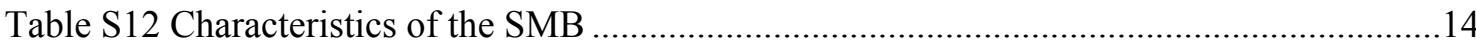

Table S13 Parameters for the multicomponent Langmuir isotherm ( $\left.q i=q_{2 m} \underline{i K i C p i} \underline{1} \pm \underline{K i C p i}\right)$ from Silva et al. ${ }^{4}$.

Table S14 Comparison of the TMB against the simulation of the industrial-scale unit from Silva

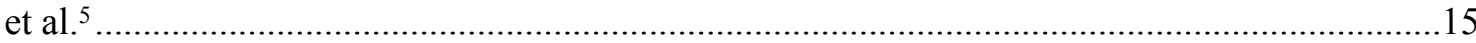

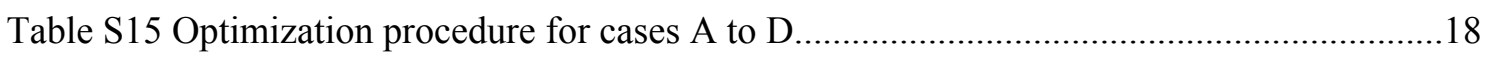

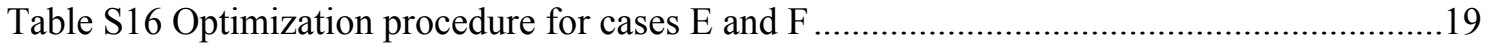

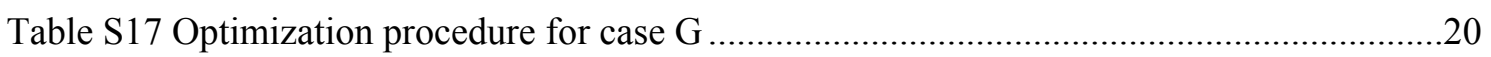


Table S18 Optimization of the current aromatics complex (A)

Table S19 Mass balance for the current aromatics complex base case (A0) ...............................22

Table S20 Mass balance for the current aromatics complex after step 1 (A1) .............................23

Table S21 Mass balance for the current aromatics complex after step 2 (A2) ............................24

Table S22 Mass balance for the current aromatics complex after step 3 (A3) .............................25

Table S23 Mass balance for the current aromatics complex after step 4 (A4) …..........................26

Table S24 Mass balance for the current aromatics complex after step 5 (A5) ............................27

Table S25 Optimization of the modified aromatics complex with separation of naphthenes

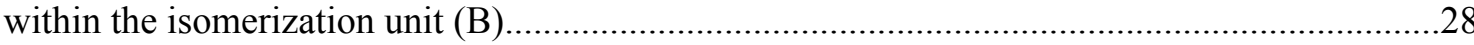

Table S26 Mass balance for the modified aromatics complex with separation of naphthenes within the isomerization unit after step 1 (B1)

Table S27 Mass balance for the modified aromatics complex with separation of naphthenes

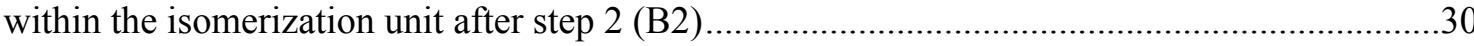

Table S28 Mass balance for the modified aromatics complex with separation of naphthenes within the isomerization unit after step 3 (B3)

Table S29 Mass balance for the modified aromatics complex with separation of naphthenes within the isomerization unit after step 4 (B4).

Table S30 Mass balance for the modified aromatics complex with separation of naphthenes

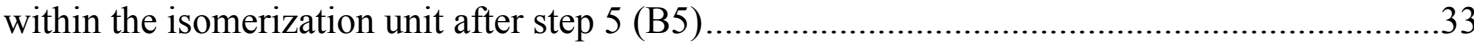

Table S31 Optimization of the modified aromatics complex with crystallization at $218 \mathrm{~K}$ (C)..34 Table S32 Mass balance for the modified aromatics complex with crystallization at $218 \mathrm{~K}$ after step $1(\mathrm{C} 1)$

Table S33 Mass balance for the modified aromatics complex with crystallization at $218 \mathrm{~K}$ after step 2 (C2)

Table S34 Mass balance for the modified aromatics complex with crystallization at $218 \mathrm{~K}$ after step 3 (C3)

Table S35 Mass balance for the modified aromatics complex with crystallization at $218 \mathrm{~K}$ after step 4 (C4)

Table S36 Mass balance for the modified aromatics complex with crystallization at $218 \mathrm{~K}$ after step 5 (C5)

Table S37 Optimization of the modified aromatics complex with crystallization at $244 \mathrm{~K}$ (D) .40 Table S38 Mass balance for the modified aromatics complex with crystallization at $244 \mathrm{~K}$ after step 1 (D1)

Table S39 Mass balance for the modified aromatics complex with crystallization at $244 \mathrm{~K}$ after step 2 (D2)

Table S40 Mass balance for the modified aromatics complex with crystallization at $244 \mathrm{~K}$ after step 3 (D3)

Table S41 Mass balance for the modified aromatics complex with crystallization at $244 \mathrm{~K}$ after step 4 (D4)

Table S42 Mass balance for the modified aromatics complex with crystallization at $244 \mathrm{~K}$ after step 5 (D5)

Table S43 Optimization of the modified aromatics complex with toluene methylation and crystallization at $218 \mathrm{~K}(\mathrm{E})$

Table S44 Mass balance for the modified aromatics complex with toluene methylation and crystallization at $218 \mathrm{~K}$ after step 1 (E1). 
Table S45 Mass balance for the modified aromatics complex with toluene methylation and crystallization at $218 \mathrm{~K}$ after step 2 (E2)

Table S46 Mass balance for the modified aromatics complex with toluene methylation and crystallization at $218 \mathrm{~K}$ after step 3 (E3).

Table S47 Mass balance for the modified aromatics complex with toluene methylation and crystallization at $218 \mathrm{~K}$ after step 4 (E4).

Table S48 Mass balance for the modified aromatics complex with toluene methylation and crystallization at $218 \mathrm{~K}$ after step 5 (E5)

Table S49 Optimization of the modified aromatics complex with toluene methylation and crystallization at $244 \mathrm{~K}(\mathrm{~F})$

Table S50 Mass balance for the modified aromatics complex with toluene methylation and crystallization at $244 \mathrm{~K}$ after step 1 (F1).....

Table S51 Mass balance for the modified aromatics complex with toluene methylation and crystallization at $244 \mathrm{~K}$ after step 2 (F2).

Table S52 Mass balance for the modified aromatics complex with toluene methylation and crystallization at $244 \mathrm{~K}$ after step 3 (F3)

Table S53 Mass balance for the modified aromatics complex with toluene methylation and crystallization at $244 \mathrm{~K}$ after step 4 (F4).

Table S54 Mass balance for the modified aromatics complex with toluene methylation and crystallization at $244 \mathrm{~K}$ after step 5 (F5).

Table S55 Optimization of the modified aromatics complex with toluene methylation (G) .......58

Table S56 Mass balance for the modified aromatics complex with toluene methylation after step 1 (G1)

Table S57 Mass balance for the modified aromatics complex with toluene methylation after step 2 (G2)

Table S58 Mass balance for the modified aromatics complex with toluene methylation after step 3 (G3)

Table S59 Mass balance for the modified aromatics complex with toluene methylation after step 4 (G4)

Table S60 Mass balance for the modified aromatics complex with toluene methylation after step 5 (G5) 


\section{S1. Distillation columns}

Table S1 Simulation of the benzene column in the base case (A0) using rigorous model RadFrac from commercial software Aspen

\begin{tabular}{|c|c|c|}
\hline & Distillate & Bottoms \\
\hline Flow $(\mathrm{t} / \mathrm{h})$ & 4.89 & 15.71 \\
\hline Ben $(w t \%)$ & 99.88 & 0.08 \\
\hline Tol (wt\%) & 0.12 & 99.92 \\
\hline $\mathrm{ECH}(\mathrm{wt} \%)$ & 0.00 & 0.00 \\
\hline $\mathrm{EB}(\mathrm{wt} \%)$ & 0.00 & 0.00 \\
\hline $\mathrm{PX}(\mathrm{wt} \%)$ & 0.00 & 0.00 \\
\hline MX (wt\%) & 0.00 & 0.00 \\
\hline $\mathrm{OX}(w \mathrm{t} \%)$ & 0.00 & 0.00 \\
\hline TMBen (wt\%) & 0.00 & 0.00 \\
\hline PDEB (wt\%) & 0.00 & 0.00 \\
\hline Temperature $\left({ }^{\circ} \mathrm{C}\right)$ & 91 & 140 \\
\hline Reflux ratio & \multicolumn{2}{|c|}{4.20} \\
\hline Number of stages & \multicolumn{2}{|c|}{34} \\
\hline Feed stage & \multicolumn{2}{|c|}{15} \\
\hline Condenser (GJ/h) & \multicolumn{2}{|c|}{9.77} \\
\hline Reboiler (GJ/h) & \multicolumn{2}{|c|}{9.80} \\
\hline Condenser $(\mathrm{GJ} / \mathrm{h})^{\mathrm{a}}$ & \multicolumn{2}{|c|}{9.26} \\
\hline Reboiler $(\mathrm{GJ} / \mathrm{h})^{\mathrm{a}}$ & \multicolumn{2}{|c|}{9.83} \\
\hline
\end{tabular}

a Duty obtained with the simplified model 
Table S2 Simulation of the splitter in the base case (A0) using rigorous model RadFrac from commercial software Aspen

\begin{tabular}{|c|c|c|}
\hline & Distillate & Bottoms \\
\hline Flow (t/h) & 55.28 & 7.51 \\
\hline Ben $(w t \%)$ & 0.00 & 0.00 \\
\hline Tol (wt\%) & 0.00 & 0.00 \\
\hline ECH (wt\%) & 20.51 & 0.00 \\
\hline $\mathrm{EB}(\mathrm{wt} \%)$ & 7.18 & 0.00 \\
\hline $\mathrm{PX}(w t \%)$ & 18.61 & 0.02 \\
\hline MX (wt\%) & 39.74 & 0.35 \\
\hline $\mathrm{OX}(w \mathrm{t} \%)$ & 12.84 & 25.92 \\
\hline TMBen (wt\%) & 1.12 & 72.96 \\
\hline PDEB (wt\%) & 0.00 & 0.75 \\
\hline Temperature $\left({ }^{\circ} \mathrm{C}\right)$ & 150 & 191 \\
\hline Reflux ratio & \multicolumn{2}{|c|}{0.77} \\
\hline Number of stages & \multicolumn{2}{|c|}{186} \\
\hline Feed stage & \multicolumn{2}{|c|}{18} \\
\hline Condenser (GJ/h) & \multicolumn{2}{|c|}{31.95} \\
\hline Reboiler (GJ/h) & \multicolumn{2}{|c|}{29.92} \\
\hline Condenser $(G J / h)^{\mathrm{a}}$ & \multicolumn{2}{|c|}{19.38} \\
\hline Reboiler $(\mathrm{GJ} / \mathrm{h})^{\mathrm{a}}$ & \multicolumn{2}{|c|}{20.36} \\
\hline
\end{tabular}

a Duty obtained with the simplified model 
Table S3 Simulation of the extract column in the base case (A0) using rigorous model RadFrac from commercial software Aspen

\begin{tabular}{|c|c|c|}
\hline & Distillate & Bottoms \\
\hline Flow (t/h) & 10.31 & 28.84 \\
\hline Ben (wt\%) & 0.00 & 0.00 \\
\hline Tol (wt\%) & 0.00 & 0.00 \\
\hline $\mathrm{ECH}(\mathrm{wt} \%)$ & 0.00 & 0.00 \\
\hline $\mathrm{EB}(\mathrm{wt} \%)$ & 0.00 & 0.00 \\
\hline $\mathrm{PX}(\mathrm{wt} \%)$ & 99.76 & 0.08 \\
\hline MX (wt\%) & 0.00 & 0.00 \\
\hline $\mathrm{OX}(\mathrm{wt} \%)$ & 0.11 & 0.00 \\
\hline TMBen (wt\%) & 0.00 & 0.00 \\
\hline PDEB (wt \%) & 0.13 & 99.92 \\
\hline Temperature $\left({ }^{\circ} \mathrm{C}\right)$ & 150 & 215 \\
\hline Reflux ratio & \multicolumn{2}{|c|}{2.67} \\
\hline Number of stages & \multicolumn{2}{|c|}{31} \\
\hline Feed stage & \multicolumn{2}{|c|}{13} \\
\hline Condenser (GJ/h) & \multicolumn{2}{|c|}{12.65} \\
\hline Reboiler (GJ/h) & \multicolumn{2}{|c|}{12.98} \\
\hline Condenser $(\mathrm{GJ} / \mathrm{h})^{\mathrm{a}}$ & \multicolumn{2}{|c|}{11.52} \\
\hline Reboiler $(G J / h)^{a}$ & \multicolumn{2}{|c|}{12.51} \\
\hline
\end{tabular}

a Duty obtained with the simplified model 
Table S4 Simulation of the raffinate column in the base case (A0) using rigorous model RadFrac from commercial software Aspen

\begin{tabular}{|c|c|c|}
\hline & Distillate & Bottoms \\
\hline Flow $(\mathrm{t} / \mathrm{h})$ & 45.29 & 23.38 \\
\hline Ben $(w t \%)$ & 0.00 & 0.00 \\
\hline Tol (wt\%) & 0.00 & 0.00 \\
\hline $\mathrm{ECH}(\mathrm{wt} \%)$ & 25.02 & 0.00 \\
\hline $\mathrm{EB}(\mathrm{wt} \%)$ & 8.76 & 0.00 \\
\hline $\mathrm{PX}(\mathrm{wt} \%)$ & 0.02 & 0.00 \\
\hline $\mathrm{MX}(\mathrm{wt} \%)$ & 48.53 & 0.03 \\
\hline $\mathrm{OX}(\mathrm{wt} \%)$ & 17.53 & 0.05 \\
\hline TMBen (wt\%) & 0.00 & 0.00 \\
\hline PDEB (wt\%) & 0.12 & 99.92 \\
\hline Temperature $\left({ }^{\circ} \mathrm{C}\right)$ & 149 & 215 \\
\hline Reflux ratio & \multicolumn{2}{|c|}{1.06} \\
\hline Number of stages & \multicolumn{2}{|c|}{29} \\
\hline Feed stage & \multicolumn{2}{|c|}{14} \\
\hline Condenser (GJ/h) & \multicolumn{2}{|c|}{30.45} \\
\hline Reboiler (GJ/h) & \multicolumn{2}{|c|}{29.72} \\
\hline Condenser $(\mathrm{GJ} / \mathrm{h})^{\mathrm{a}}$ & \multicolumn{2}{|c|}{30.07} \\
\hline Reboiler $(\mathrm{GJ} / \mathrm{h})^{\mathrm{a}}$ & \multicolumn{2}{|c|}{32.72} \\
\hline
\end{tabular}

a Duty obtained with the simplified model 
Table S5 Simulation of the $o$-xylene column in the base case (A0) using rigorous model RadFrac from commercial software Aspen

\begin{tabular}{|c|c|c|}
\hline & Distillate & Bottoms \\
\hline Flow (t/h) & 1.10 & 6.16 \\
\hline Ben (wt\%) & 0.00 & 0.00 \\
\hline Tol (wt\%) & 0.00 & 0.00 \\
\hline ECH (wt\%) & 0.00 & 0.00 \\
\hline $\mathrm{EB}(\mathrm{wt} \%)$ & 0.00 & 0.00 \\
\hline $\mathrm{PX}(w t \%)$ & 0.00 & 0.00 \\
\hline MX (wt\%) & 0.59 & 0.00 \\
\hline $\mathrm{OX}(\mathrm{wt} \%)$ & 99.30 & 0.09 \\
\hline TMBen (wt\%) & 0.11 & 98.99 \\
\hline PDEB (wt\%) & 0.00 & 0.92 \\
\hline Temperature $\left({ }^{\circ} \mathrm{C}\right)$ & 156 & 200 \\
\hline Reflux ratio & \multicolumn{2}{|c|}{10.86} \\
\hline Number of stages & \multicolumn{2}{|c|}{52} \\
\hline Feed stage & \multicolumn{2}{|c|}{20} \\
\hline Condenser (GJ/h) & \multicolumn{2}{|c|}{4.44} \\
\hline Reboiler (GJ/h) & \multicolumn{2}{|c|}{4.43} \\
\hline Condenser $(\mathrm{GJ} / \mathrm{h})^{\mathrm{a}}$ & \multicolumn{2}{|c|}{4.13} \\
\hline Reboiler $(G J / h)^{a}$ & \multicolumn{2}{|c|}{4.22} \\
\hline
\end{tabular}

a Duty obtained with the simplified model

\section{S2. Gas phase isomerization}

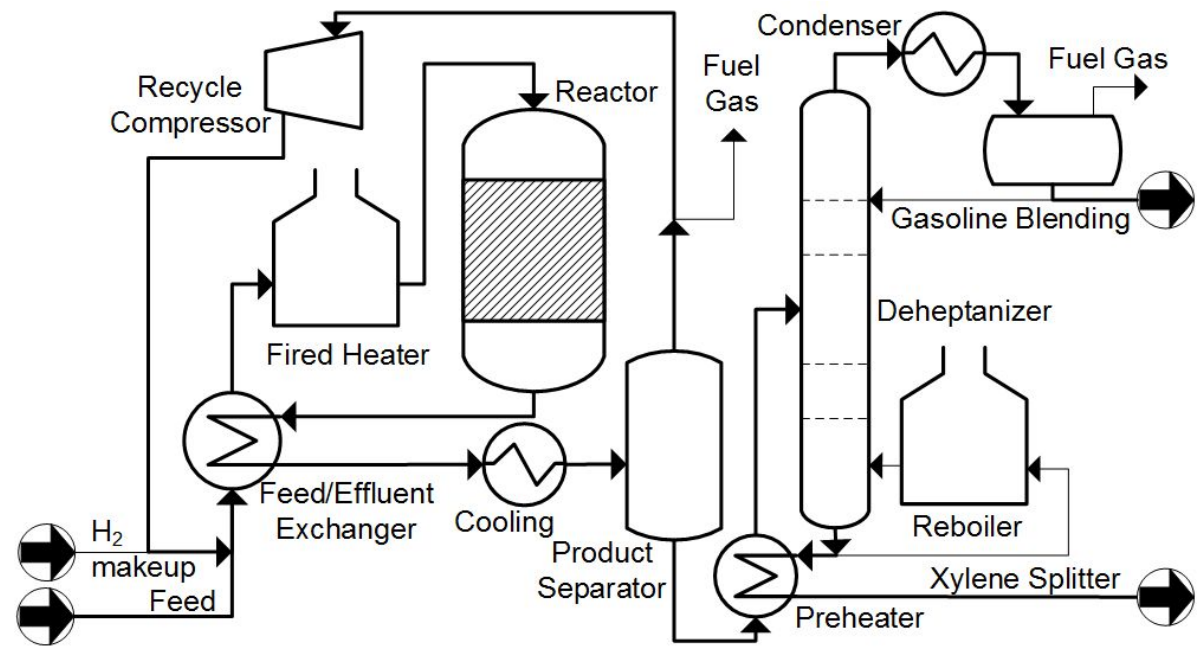

Figure S1 Simplified diagram of the isomerization unit 


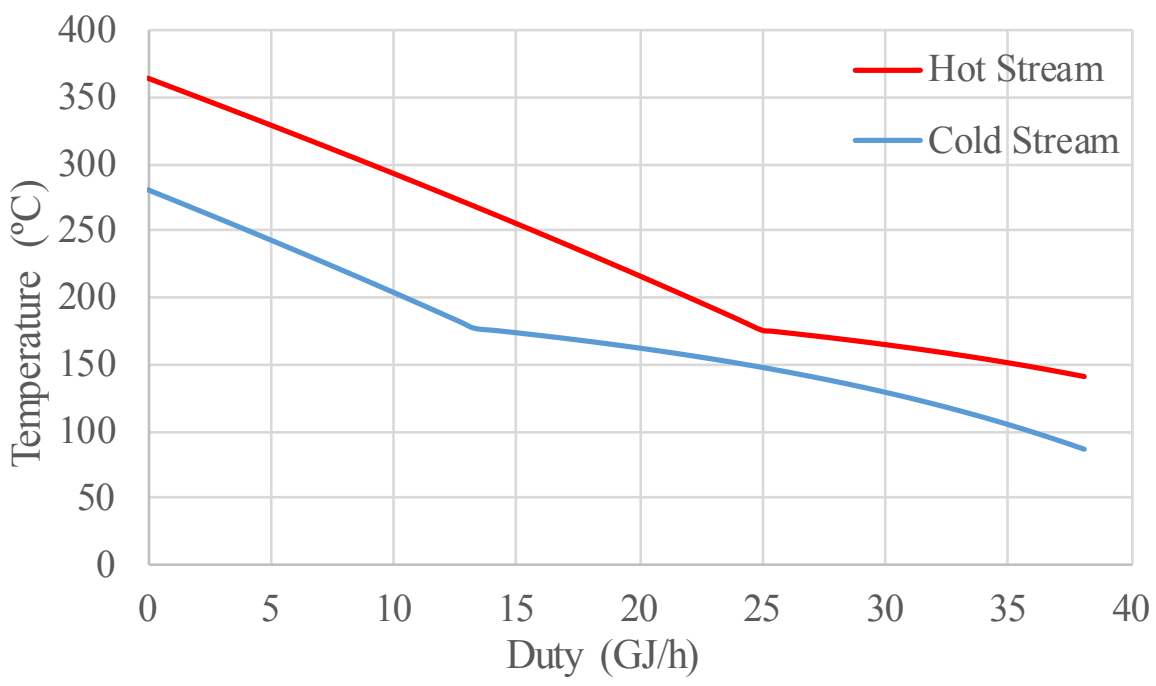

Figure S2 Temperature profile for the Feed/Effluent Exchanger for the base case A0

Table S6 Utility consumption of an Isomar unit with a capacity of 40000 BPD Parex raffinate

\begin{tabular}{lcc}
\multicolumn{1}{c}{ Utility } & UOP's Isomar & Simplified model \\
\hline Electric power & $918 \mathrm{kWh}$ & $1189 \mathrm{kWh}$ \\
High-pressure steam & $16.9 \mathrm{MT} / \mathrm{h}$ & \\
Cooling water & $236 \mathrm{~m}^{3} / \mathrm{h}$ & $112 \mathrm{GJ} / \mathrm{h}$ \\
Fuel oil & $20.8 \times 10^{6} \mathrm{kcal} / \mathrm{h}$ & $116 \mathrm{GJ} / \mathrm{h}$ \\
\hline Total cost & $1802 \$ / \mathrm{h}$ & $1759 \$ / \mathrm{h}$ \\
\hline
\end{tabular}

\section{S3. Crystallization}

Table S7 Utility consumption of a Crystallization unit with a capacity of 350000 ton/year p-xylene ${ }^{\mathrm{a}}$

\begin{tabular}{lcc}
\hline \multicolumn{1}{c}{ Utility } & ExxonMobil's Crystallizer ${ }^{2}$ & Simplified model \\
\hline Electric power & $3505 \mathrm{kWh}$ & \\
Cooling water & $300 \mathrm{~m}^{3} / \mathrm{h}$ & $20.8 \mathrm{GJ} / \mathrm{h}$ \\
Refrigerant $\left(-34^{\circ} \mathrm{C}\right)$ & & $211 \$ / \mathrm{h}$ \\
\hline Total cost & $215 \$ / \mathrm{h}$ &
\end{tabular}

a $85 \mathrm{wt} \%$ PX stream as feed 


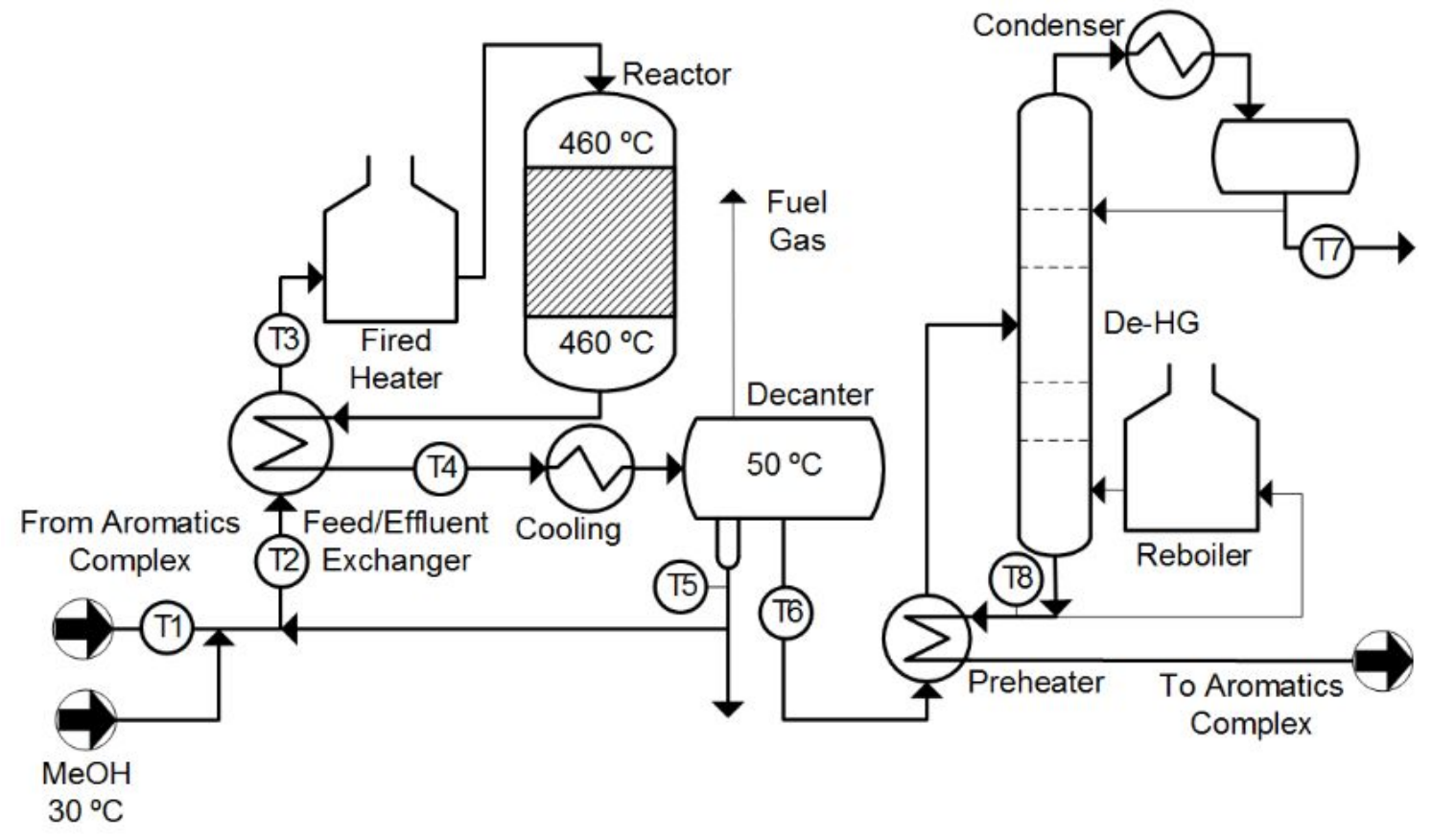

Figure S3 Simplified diagram of the toluene methylation unit

$$
\begin{aligned}
& \text { Tol }+\mathrm{MeOH} \leftrightarrow \mathrm{PX}+\text { Water } \quad R_{1}=k_{1} P_{\mathrm{Tol}} P_{\mathrm{MeOH}} \quad R_{-1}=\frac{k_{1}}{K_{e q, 1}} P_{P X} P_{\text {Water }} \\
& 2 \mathrm{MeOH} \leftrightarrow \mathrm{FG}+2 \text { Water } \quad R_{2}=k_{2} P_{\mathrm{MeOH}}^{2} \quad R_{-2}=\frac{k_{2}}{K_{e q, 2}} P_{F G} P_{\text {Water }}^{2} \\
& \text { Tol } \rightarrow \frac{1}{2} \text { Ben }+\frac{1}{2} P X \quad R_{3}=\eta_{3} k_{3} P_{T o l} \\
& P X \rightarrow T o l+\frac{1}{2} F G \quad R_{4}=\eta_{4} k_{4} P_{P X} \\
& P X \rightarrow \frac{1}{2} M X+\frac{1}{2} O X \quad R_{5}=k_{5} P_{P X}
\end{aligned}
$$

$\underline{\text { Table S8 Kinetic parameters for toluene methylation from Valverde }}{ }^{3}$

\begin{tabular}{ll}
\hline$k_{1}$ & $224 \mathrm{~mol} / \mathrm{kg}_{\mathrm{cat}} \mathrm{atm}^{2} \mathrm{~h}$ \\
$k_{2}$ & $334 \mathrm{~mol} / \mathrm{kg}_{\mathrm{cat}} \mathrm{atm}^{2} \mathrm{~h}$ \\
$k_{3}$ & $6.02 \mathrm{~mol} / \mathrm{kg}_{\mathrm{cat}} \mathrm{atmh}$ \\
$k_{4}$ & $15.3 \mathrm{~mol} / \mathrm{kg}_{\mathrm{cat}} \mathrm{atmh}$ \\
$k_{5}$ & $15.4 \mathrm{~mol} / \mathrm{kg}_{\mathrm{cat}} \mathrm{atmh}$ \\
$\eta_{3}$ & 0.778 \\
$\eta_{4}$ & 2.977
\end{tabular}


Table S9 Mass balance for the toluene methylation unit after step 5 of the optimization of the modified aromatics complex with toluene methylation and crystallization at $218 \mathrm{~K}$ (E5)

\begin{tabular}{lcccccccc}
\hline Stream & T1 & T2 & T3 & T4 & T5 & T6 & T7 & T8 \\
\hline Flow (t/h) & 76.07 & 98.57 & 98.57 & 98.57 & 18.01 & 78.71 & 1.20 & 77.51 \\
Fuel Gas (wt\%) & 0.00 & 0.00 & 0.00 & 1.87 & 0.00 & 0.00 & 0.00 & 0.00 \\
Ben (wt\%) & 0.10 & 0.09 & 0.09 & 0.72 & 0.08 & 0.89 & 53.09 & 0.08 \\
Tol (wt\%) & 99.81 & 77.11 & 77.11 & 61.26 & 0.77 & 76.54 & 0.20 & 77.73 \\
ECH (wt\%) & 0.00 & 0.00 & 0.00 & 0.00 & 0.00 & 0.00 & 0.00 & 0.00 \\
EB (wt\%) & 0.00 & 0.00 & 0.00 & 0.00 & 0.00 & 0.00 & 0.00 & 0.00 \\
PX (wt\%) & 0.09 & 0.08 & 0.08 & 16.50 & 0.08 & 20.64 & 0.00 & 20.96 \\
MX (wt\%) & 0.00 & 0.00 & 0.00 & 0.49 & 0.00 & 0.61 & 0.00 & 0.62 \\
OX (wt\%) & 0.00 & 0.00 & 0.00 & 0.49 & 0.00 & 0.61 & 0.00 & 0.62 \\
TMBen (wt\%) & 0.00 & 0.00 & 0.00 & 0.00 & 0.00 & 0.00 & 0.00 & 0.00 \\
PDEB (wt\%) & 0.00 & 0.00 & 0.00 & 0.00 & 0.00 & 0.00 & 0.00 & 0.00 \\
MeOH (wt\%) & 0.00 & 15.77 & 15.77 & 6.51 & 32.60 & 0.69 & 45.06 & 0.00 \\
Water (wt\%) & 0.00 & 6.95 & 6.95 & 12.16 & 66.46 & 0.03 & 1.65 & 0.00 \\
\hline Temp. $\left({ }^{\circ} \mathrm{C}\right)$ & 125 & 98 & 200 & 132 & 50 & 50 & 78 & 142 \\
\hline
\end{tabular}

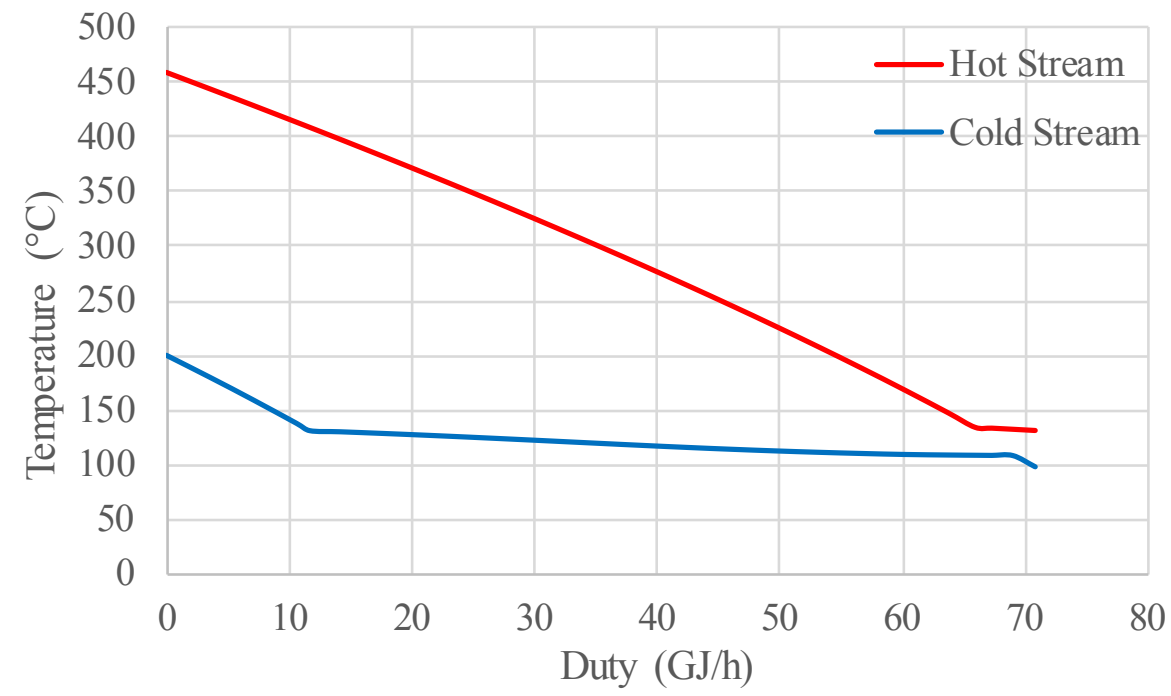

Figure S4 Temperature profile of the Feed/Effluent Exchanger after step 5 of the optimization of the modified aromatics complex with toluene methylation and crystallization at $218 \mathrm{~K}$ (E5) 
Table S10 Mass balance for the toluene methylation unit after step 5 of the optimization of the modified aromatics complex with toluene methylation and crystallization at $244 \mathrm{~K}$ (F5)

\begin{tabular}{lcccccccc}
\hline Stream & T1 & T2 & T3 & T4 & T5 & T6 & T7 & T8 \\
\hline Flow (t/h) & 75.97 & 98.44 & 98.44 & 98.44 & 17.99 & 78.61 & 1.20 & 77.41 \\
Fuel Gas (wt\%) & 0.00 & 0.00 & 0.00 & 1.87 & 0.00 & 0.00 & 0.00 & 0.00 \\
Ben (wt\%) & 0.10 & 0.09 & 0.09 & 0.73 & 0.08 & 0.89 & 53.21 & 0.08 \\
Tol (wt\%) & 99.81 & 77.11 & 77.11 & 61.24 & 0.77 & 76.52 & 0.20 & 77.70 \\
ECH (wt\%) & 0.00 & 0.00 & 0.00 & 0.00 & 0.00 & 0.00 & 0.00 & 0.00 \\
EB (wt\%) & 0.00 & 0.00 & 0.00 & 0.00 & 0.00 & 0.00 & 0.00 & 0.00 \\
PX (wt\%) & 0.09 & 0.08 & 0.08 & 16.51 & 0.08 & 20.65 & 0.00 & 20.97 \\
MX (wt\%) & 0.00 & 0.00 & 0.00 & 0.49 & 0.00 & 0.61 & 0.00 & 0.62 \\
OX (wt\%) & 0.00 & 0.00 & 0.00 & 0.49 & 0.00 & 0.61 & 0.00 & 0.62 \\
TMBen (wt\%) & 0.00 & 0.00 & 0.00 & 0.00 & 0.00 & 0.00 & 0.00 & 0.00 \\
PDEB (wt\%) & 0.00 & 0.00 & 0.00 & 0.00 & 0.00 & 0.00 & 0.00 & 0.00 \\
MeOH (wt\%) & 0.00 & 15.77 & 15.77 & 6.50 & 32.60 & 0.69 & 44.94 & 0.00 \\
Water $(w t \%)$ & 0.00 & 6.95 & 6.95 & 12.16 & 66.46 & 0.03 & 1.65 & 0.00 \\
\hline Temp. $\left({ }^{\circ} \mathrm{C}\right)$ & 125 & 98 & 200 & 132 & 50 & 50 & 78 & 142 \\
\hline
\end{tabular}

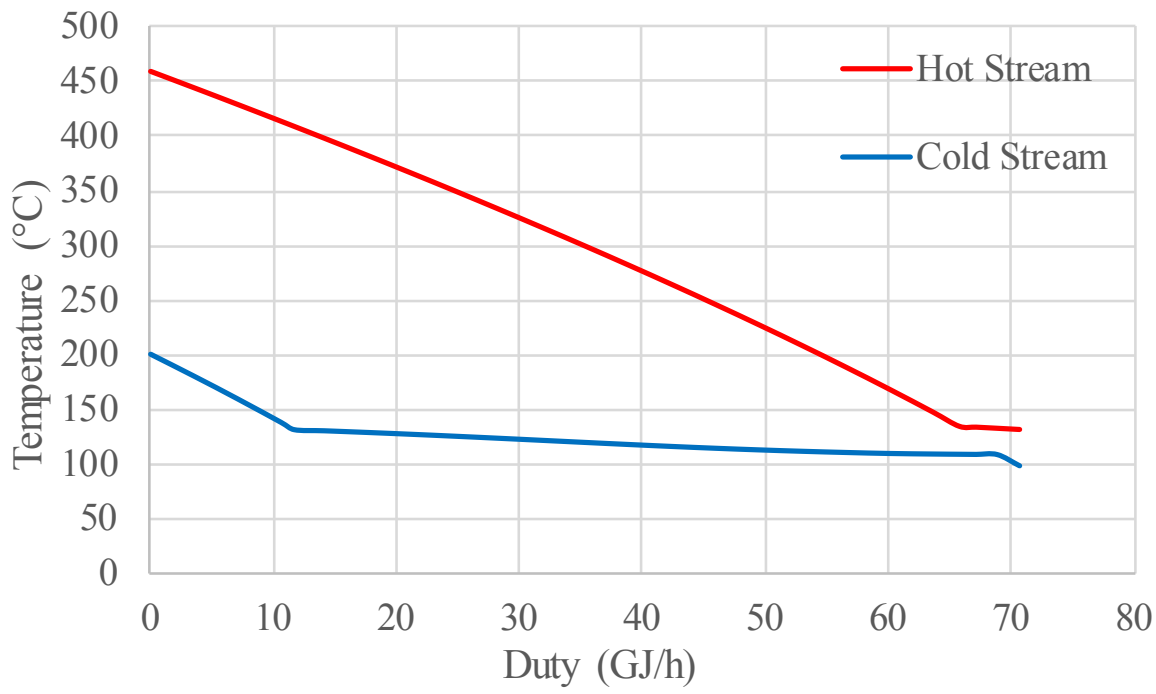

Figure S5 Temperature profile of the Feed/Effluent Exchanger after step 5 of the optimization of the modified aromatics complex with toluene methylation and crystallization at $244 \mathrm{~K}$ (F5) 
Table S11 Simulation of the distillation column within the toluene methylation unit after step 5 (E5 and F5) using rigorous model RadFrac from commercial software Aspen

\begin{tabular}{|c|c|c|c|c|}
\hline & \multicolumn{2}{|c|}{ E5 } & \multicolumn{2}{|c|}{ F5 } \\
\hline & Distillate & Bottom & Distillate & Bottom \\
\hline Flow $(\mathrm{t} / \mathrm{h})$ & 1.19 & 77.52 & 1.19 & 77.42 \\
\hline Ben (wt $\%)$ & 52.69 & 0.09 & 53.51 & 0.07 \\
\hline Tol (wt\%) & 0.20 & 77.72 & 0.20 & 77.70 \\
\hline $\mathrm{PX}(\mathrm{wt} \%)$ & 0.00 & 20.96 & 0.00 & 20.98 \\
\hline MX (wt\%) & 0.00 & 0.61 & 0.00 & 0.62 \\
\hline $\mathrm{OX}(\mathrm{wt} \%)$ & 0.00 & 0.61 & 0.00 & 0.62 \\
\hline $\mathrm{MeOH}(\mathrm{wt} \%)$ & 45.45 & 0.00 & 44.65 & 0.00 \\
\hline Water (wt\%) & 1.66 & 0.00 & 1.63 & 0.00 \\
\hline Temperature $\left({ }^{\circ} \mathrm{C}\right)$ & 74 & 144 & 74 & 144 \\
\hline Reflux ratio & \multicolumn{2}{|c|}{27.85} & \multicolumn{2}{|c|}{29.24} \\
\hline Number of stages & \multicolumn{2}{|c|}{30} & \multicolumn{2}{|c|}{31} \\
\hline Feed stage & \multicolumn{2}{|c|}{23} & \multicolumn{2}{|c|}{24} \\
\hline Condenser (GJ/h) & \multicolumn{2}{|c|}{25.34} & \multicolumn{2}{|c|}{26.75} \\
\hline Reboiler (GJ/h) & \multicolumn{2}{|c|}{27.13} & \multicolumn{2}{|c|}{27.32} \\
\hline Condenser $(G J / h)^{a}$ & \multicolumn{2}{|c|}{25.72} & \multicolumn{2}{|c|}{25.68} \\
\hline Reboiler $(\mathrm{GJ} / \mathrm{h})^{\mathrm{a}}$ & \multicolumn{2}{|c|}{24.90} & \multicolumn{2}{|c|}{24.86} \\
\hline
\end{tabular}

a Duty obtained with the simplified model

\section{S5. Simulated Moving Bed}

Table S12 Characteristics of the SMB

\begin{tabular}{lc}
\hline Number of columns & 24 \\
Column length $(\mathrm{m})$ & 1.14 \\
Column diameter $(\mathrm{m})$ & 4.12 \\
Bed porosity & 0.39 \\
Particle radius $(\mathrm{mm})$ & 0.31 \\
Particle apparent density $\left(\mathrm{kg} / \mathrm{m}^{3}\right)$ & 1480 \\
Particle porosity & 0.36 \\
Tortuosity factor & 7 \\
\hline
\end{tabular}


Table S13 Parameters for the multicomponent Langmuir isotherm

\begin{tabular}{lcc}
$\left(q_{i}=q_{m, i} K_{i} \overline{C_{p i}} /\left(1+\sum K_{i} \overline{C_{p i}}\right)\right)$ from Silva et al. ${ }^{4}$ & $K_{i}\left(\mathrm{~m}^{3} / \mathrm{kg}\right)$ & $q_{m, i}(\mathrm{~kg} / \mathrm{kg})$ \\
\hline$p$-Xylene & 2.781 & 0.133 \\
$m$-Xylene & 0.196 & 0.128 \\
$o$-Xylene & 0.240 & 0.130 \\
Ethylbenzene & 0.365 & 0.125 \\
$p$-Diethylbenzene & 6.408 & 0.094 \\
Naphthenes & 0 & 0 \\
\hline
\end{tabular}

Table S14 Comparison of the TMB against the simulation of the industrial-scale unit from Silva et al. ${ }^{5}$

\begin{tabular}{lcccccc}
\hline & \multicolumn{3}{c}{ SMB from Silva et al. } & \multicolumn{3}{c}{ Simplified TMB model } \\
\hline & Run 1 & Run 2 & Run 3 & Run 1 & Run 2 & Run 3 \\
\hline Switching time $(\mathrm{s})$ & 112.1 & 89.7 & 106.3 & 112.1 & 89.7 & 106.3 \\
Desorbent $\left(\mathrm{m}^{3} / \mathrm{h}\right)$ & 130.39 & 163.04 & 140 & 130.39 & 163.04 & 139.63 \\
Extract $\left(\mathrm{m}^{3} / \mathrm{h}\right)$ & 54.21 & 69.59 & 60 & 54.21 & 69.59 & 60.17 \\
Raffinate $\left(\mathrm{m}^{3} / \mathrm{h}\right)^{\mathrm{a}}$ & 182.81 & 226.11 & 190 & 175.58 & 217.09 & 181.59 \\
Feed $\left(\mathrm{m}^{3} / \mathrm{h}\right)$ & 99.40 & 123.63 & 102 & 99.40 & 123.63 & 102.13 \\
Extract purity $(\mathrm{wt} \%)$ & 99.76 & 99.53 & 99.64 & 99.99 & 99.94 & 100.0 \\
Recovery $(\%)$ & 97.64 & 97.7 & 97.69 & 99.99 & 99.99 & 99.99 \\
Desorbent consumption $(\mathrm{kg} / \mathrm{kg})$ & 7.33 & 7.36 & 7.61 & 7.09 & 7.13 & 7.38 \\
Productivity $\left(\mathrm{kg} / \mathrm{hm}^{3}\right)$ & 67.87 & 84.49 & 69.98 & 60.01 & 74.63 & 61.78 \\
\hline a The difference between the two models is due to the flushings & & &
\end{tabular}

a The difference between the two models is due to the flushings 

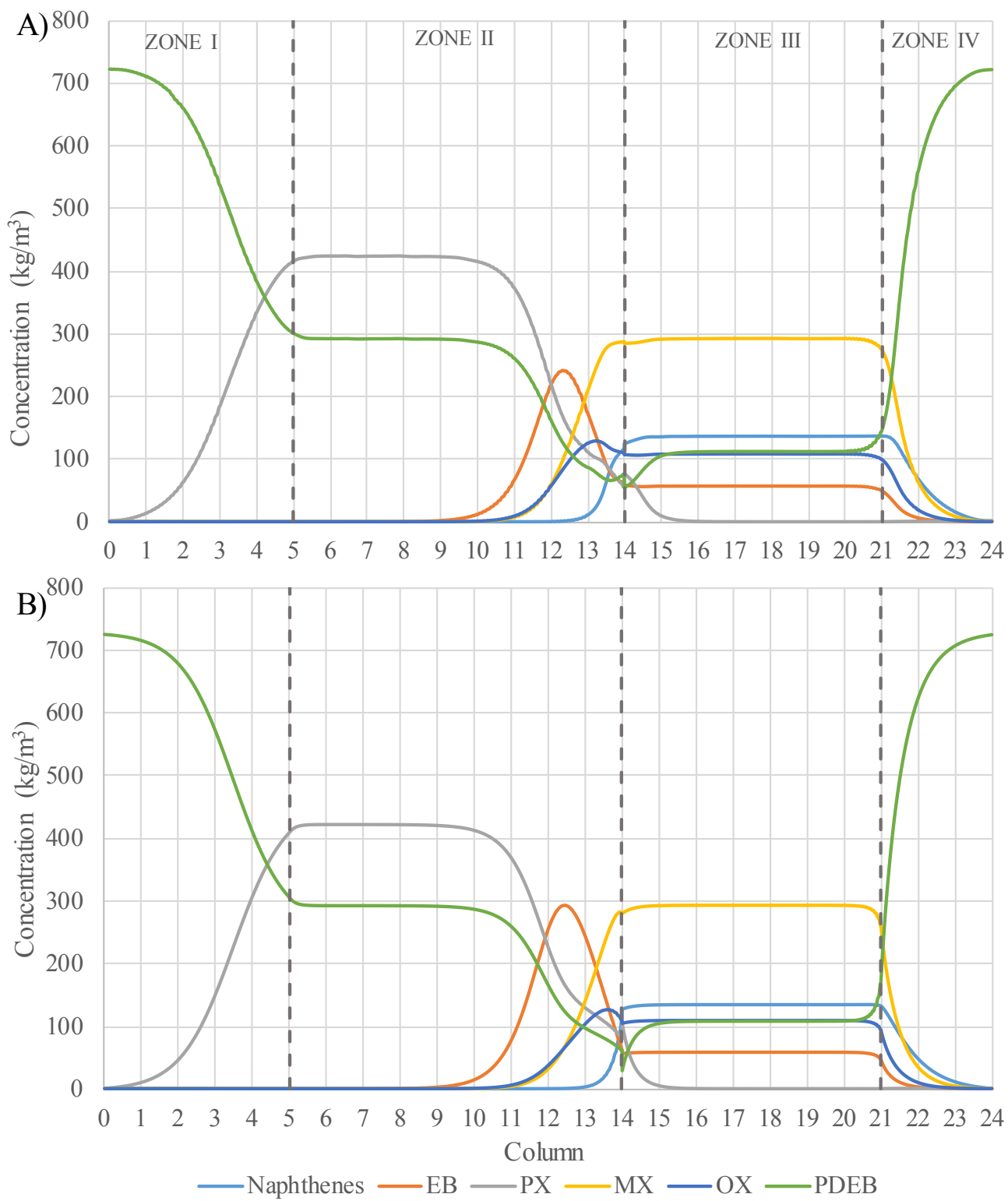

Figure S6 Internal concentration profile of the SMB unit for the case A after step 4 (A4): A) calculated as SMB at the middle of the switching time after the cycle steady state was reached and B) calculated using the TMB approach 


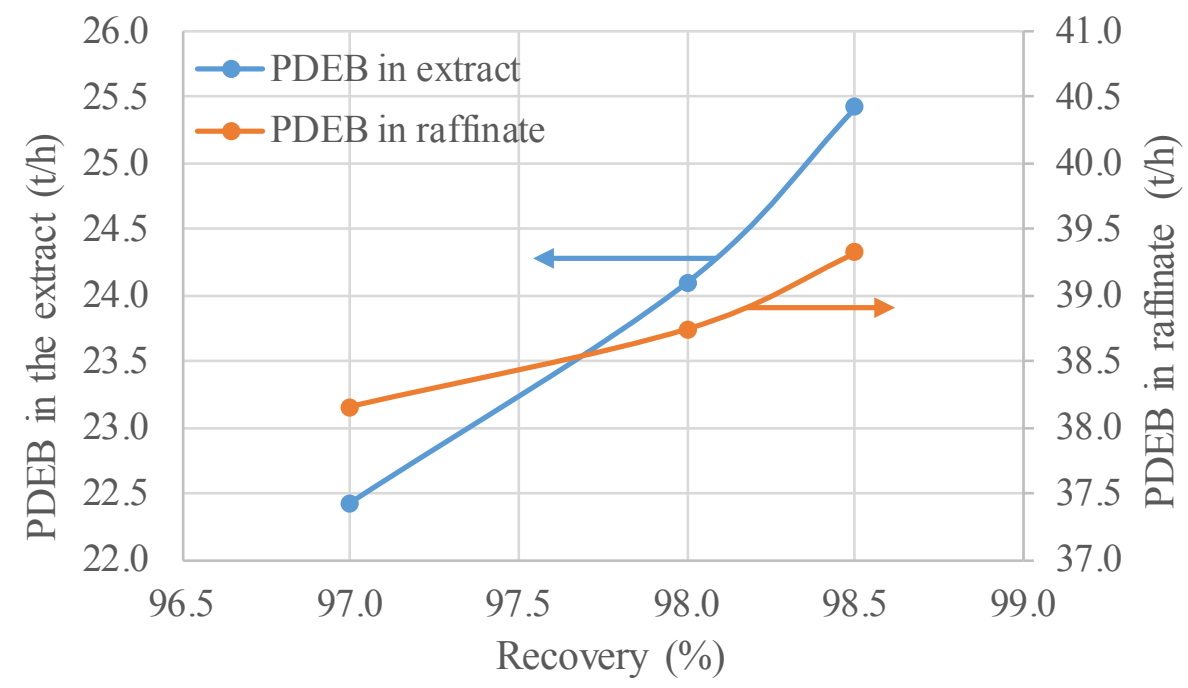

Figure S7 Desorbent ( $p$-diethylbenzene - PDEB) in the extract and raffinate for fixed feed (62.3 $\left.\mathrm{m}^{3} / \mathrm{h}\right)$ and extract purity $(99.82 \mathrm{wt} \%)$ and several recovery ratios

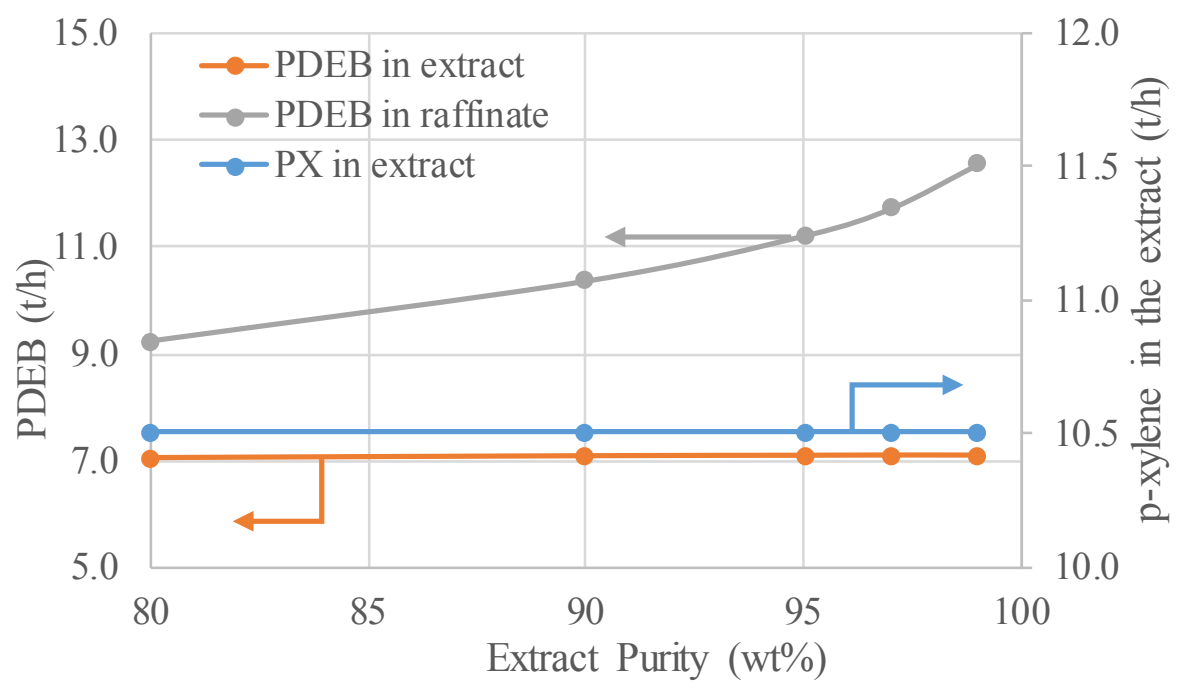

Figure S8 Desorbent ( $p$-diethylbenzene - PDEB) in the extract and raffinate and p-xylene (PX) in the extract for fixed feed $\left(80.3 \mathrm{~m}^{3} / \mathrm{h}\right)$ and recovery $(99.987 \%)$ and several recovery ratios 


\section{S6. Optimization}

Table S15 Optimization procedure for cases A to D

\begin{tabular}{|c|c|c|c|c|}
\hline Step & $\begin{array}{l}\text { Objective } \\
\text { Function }\end{array}$ & Decision Variables $(\beta)$ & Fixed Variables & Constraints \\
\hline 1 & $\max _{\beta}\{$ Profit $\}$ & $\begin{array}{l}Q_{1} \\
Q_{D} \\
Q_{X}\end{array}$ & $\begin{array}{c}\text { Stream } 1 \\
\text { Stream } 12 \\
t_{s} \\
N_{\mathrm{I}} \mathrm{Col} \\
N_{\mathrm{II}} \mathrm{Col} \\
N_{\mathrm{IV}} \mathrm{Col} \\
\end{array}$ & $\begin{array}{c}\sum_{k} N_{k}^{C o l}=24 \\
\mathrm{PX}_{\text {Stream } 11} \geq 99.7 \mathrm{wt} \% \\
\Delta \mathrm{P}_{\mathrm{SMB}} \leq 685 \mathrm{kPa} \\
\Delta \mathrm{P} / \mathrm{bed}_{\mathrm{SMB}} \leq 35 \mathrm{kPa} \\
\text { Recovery }_{\mathrm{SMB}} \geq 97 \%\end{array}$ \\
\hline 2 & $\max _{\beta}\{$ Profit $\}$ & $\begin{array}{c}Q_{1} \\
Q_{D} \\
Q_{X} \\
N_{\mathrm{I}} \mathrm{Col} \\
N_{\mathrm{II}}{ }^{\mathrm{Col}} \\
N_{\mathrm{IV}}{ }^{\mathrm{Col}} \\
\end{array}$ & $\begin{array}{c}\text { Stream } 1 \\
\text { Stream } 12 \\
t_{s}\end{array}$ & $\begin{array}{c}\sum_{k} N_{k}^{C o l}=24 \\
\mathrm{PX}_{\text {Stream } 11} \geq 99.7 \mathrm{wt} \% \\
\Delta \mathrm{P}_{\mathrm{SMB}} \leq 685 \mathrm{kPa} \\
\Delta \mathrm{P} / \text { bed }_{\mathrm{SMB}} \leq 35 \mathrm{kPa} \\
\text { Recovery }_{\mathrm{SMB}} \geq 97 \%\end{array}$ \\
\hline 3 & $\max _{\beta}\{$ Profit $\}$ & $\begin{array}{l}Q_{1} \\
Q_{D} \\
Q_{X} \\
t_{s}\end{array}$ & $\begin{array}{c}\text { Stream } 1 \\
\text { Stream } 12 \\
N_{\mathrm{I}} \mathrm{Col} \\
N_{\mathrm{II}} \mathrm{Col} \\
N_{\mathrm{IV}}{ }^{\mathrm{Col}}\end{array}$ & $\begin{array}{c}\sum_{k} N_{k}^{C o l}=24 \\
\mathrm{PX}_{\text {Stream } 11} \geq 99.7 \mathrm{wt} \% \\
\Delta \mathrm{P}_{\mathrm{SMB}} \leq 685 \mathrm{kPa} \\
\Delta \mathrm{P} / \text { bed }_{\mathrm{SMB}} \leq 35 \mathrm{kPa} \\
\text { Recovery }_{\mathrm{SMB}} \geq 97 \%\end{array}$ \\
\hline 4 & $\max _{\beta}\{$ Profit $\}$ & $\begin{array}{l}Q_{1} \\
Q_{D} \\
Q_{X} \\
t_{s}\end{array}$ & $\begin{array}{c}\text { Stream } 1 \\
\text { Stream } 12 \\
N_{\mathrm{I}} \mathrm{Col} \\
N_{\mathrm{II}} \mathrm{Col} \\
N_{\mathrm{IV}} \mathrm{Col}\end{array}$ & $\begin{array}{c}\sum_{k} N_{k}^{C o l}=24 \\
\mathrm{PX}_{\text {Stream } 11} \geq 99.7 \mathrm{wt} \% \\
\Delta \mathrm{P}_{\mathrm{SMB}} \leq 685 \mathrm{kPa} \\
\Delta \mathrm{P} / \text { bed }_{\mathrm{SMB}} \leq 35 \mathrm{kPa} \\
\text { Recovery }_{\mathrm{SMB}} \geq 97 \%\end{array}$ \\
\hline 5 & $\max _{\beta}\{$ Profit $\}$ & $\begin{array}{c}Q_{1} \\
Q_{D} \\
Q_{X} \\
t_{s} \\
N_{\mathrm{I}}{ }^{\mathrm{Col}} \\
N_{\mathrm{II}}{ }^{\mathrm{Col}} \\
N_{\mathrm{IV}}{ }^{\mathrm{Col}}\end{array}$ & $\begin{array}{l}\text { Stream } 1 \\
\text { Stream } 12\end{array}$ & $\begin{array}{c}\sum_{k} N_{k}^{C o l}=24 \\
\mathrm{PX}_{\text {Stream } 11} \geq 99.7 \mathrm{wt} \% \\
\Delta \mathrm{P}_{\mathrm{SMB}} \leq 685 \mathrm{kPa} \\
\Delta \mathrm{P} / \text { bed }_{\mathrm{SMB}} \leq 35 \mathrm{kPa} \\
\text { Recovery }_{\mathrm{SMB}} \geq 97 \%\end{array}$ \\
\hline
\end{tabular}


Table S16 Optimization procedure for cases $\mathrm{E}$ and $\mathrm{F}$

\begin{tabular}{|c|c|c|c|c|}
\hline Step & $\begin{array}{l}\text { Objective } \\
\text { Function }\end{array}$ & Decision Variables $(\beta)$ & Fixed Variables & Constraints \\
\hline 1 & $\max _{\beta}\{$ Profit $\}$ & $\begin{array}{c}Q_{\mathrm{I}} \\
Q_{D} \\
Q_{X} \\
\text { Tol/MeOH } \\
\text { Water/HC } \\
\text { Space time }\end{array}$ & $\begin{array}{c}\text { Stream } 1 \\
\text { Recovery OX } \\
t_{s} \\
N_{\mathrm{I}} \mathrm{Col} \\
N_{\mathrm{II}} \mathrm{Col} \\
N_{\mathrm{IV}}{ }^{\mathrm{Col}}\end{array}$ & $\begin{array}{c}\sum_{k} N_{k}^{C o l}=24 \\
\mathrm{PX}_{\text {Stream } 20} \geq 75 \mathrm{wt} \% \\
\Delta \mathrm{P}_{\mathrm{SMB}} \leq 685 \mathrm{kPa} \\
\Delta \mathrm{P} / \text { bed }_{\mathrm{SMB}} \leq 35 \mathrm{kPa} \\
\text { Recovery } \\
\text { MeOH } \geq 97 \% \\
\text { MeOH loss } \leq 40 \%\end{array}$ \\
\hline 2 & $\max _{\beta}\{$ Profit $\}$ & $\begin{array}{c}Q_{\mathrm{I}} \\
Q_{D} \\
Q_{X} \\
N_{\mathrm{I}} \mathrm{Col} \\
N_{\mathrm{II}} \mathrm{Col} \\
N_{\mathrm{IV}}{ }^{C o l} \\
\text { Tol/MeOH } \\
\text { Water/HC } \\
\text { Space time }\end{array}$ & $\begin{array}{c}\text { Stream } 1 \\
\text { Recovery OX } \\
t_{s}\end{array}$ & $\begin{array}{c}\sum_{k} N_{k}^{C o l}=24 \\
\mathrm{PX}_{\text {Stream } 20} \geq 75 \mathrm{wt} \% \\
\Delta \mathrm{P}_{\mathrm{SMB}} \leq 685 \mathrm{kPa} \\
\Delta \mathrm{P}_{\text {bed }_{\mathrm{SMB}}} \leq 35 \mathrm{kPa} \\
\text { Recovery } \\
\text { MeOH loss } \leq 97 \% \\
\end{array}$ \\
\hline 3 & $\max _{\beta}\{$ Profit $\}$ & $\begin{array}{c}Q_{\mathrm{I}} \\
Q_{D} \\
Q_{X} \\
t_{s} \\
\text { Tol/MeOH } \\
\text { Water/HC } \\
\text { Space time }\end{array}$ & $\begin{array}{c}\text { Stream } 1 \\
\text { Recovery OX } \\
N_{\mathrm{I}} \mathrm{Col} \\
N_{\mathrm{II}} \mathrm{Col} \\
N_{\mathrm{IV}}{ }^{\mathrm{Col}}\end{array}$ & $\begin{array}{c}\sum_{k} N_{k}^{C o l}=24 \\
\mathrm{PX}_{\text {Stream } 20} \geq 75 \mathrm{wt} \% \\
\Delta \mathrm{P}_{\mathrm{SMB}} \leq 685 \mathrm{kPa} \\
\Delta \mathrm{P} / \text { bed }_{\mathrm{SMB}} \leq 35 \mathrm{kPa} \\
\text { Recovery } \\
\text { MeOB } \\
\text { MeOH loss } \leq 47 \%\end{array}$ \\
\hline 4 & $\max _{\beta}\{$ Profit $\}$ & $\begin{array}{c}Q_{\mathrm{I}} \\
Q_{D} \\
Q_{X} \\
t_{s} \\
\text { Tol/MeOH } \\
\text { Water/HC } \\
\text { Space time } \\
\end{array}$ & $\begin{array}{c}\text { Stream } 1 \\
\text { Recovery OX } \\
N_{\mathrm{I}} \mathrm{Col} \\
N_{\mathrm{II}} \mathrm{Col} \\
N_{\mathrm{IV}}{ }^{\mathrm{Col}}\end{array}$ & $\begin{array}{c}\sum_{k} N_{k}^{C o l}=24 \\
\mathrm{PX}_{\text {Stream } 20} \geq 75 \mathrm{wt} \% \\
\Delta \mathrm{P}_{\mathrm{SMB}} \leq 685 \mathrm{kPa} \\
\Delta \mathrm{P} / \text { bed }_{\mathrm{SMB}} \leq 35 \mathrm{kPa} \\
\text { Recovery } \\
\text { MeOH } \\
\text { MeOH loss } \leq 47 \%\end{array}$ \\
\hline 5 & $\max _{\beta}\{$ Profit $\}$ & $\begin{array}{c}Q_{\mathrm{I}} \\
Q_{D} \\
Q_{X} \\
t_{s} \\
N_{\mathrm{I}} \mathrm{Col} \\
N_{\mathrm{II}} \mathrm{Col} \\
N_{\mathrm{IV}}{ }^{C o l} \\
\text { Tol/MeOH } \\
\text { Water/HC } \\
\text { Space time }\end{array}$ & $\begin{array}{c}\text { Stream } 1 \\
\text { Recovery OX }\end{array}$ & $\begin{array}{c}\sum_{k} N_{k}^{C o l}=24 \\
\mathrm{PX}_{\text {Stream } 20} \geq 75 \mathrm{wt} \% \\
\Delta \mathrm{P}_{\mathrm{SMB}} \leq 685 \mathrm{kPa} \\
\Delta \mathrm{P} / \text { bed }_{\mathrm{SMB}} \leq 35 \mathrm{kPa} \\
\text { Recovery } \\
\text { MeOH } \geq 97 \% \\
\text { MeOHs } \leq 40 \%\end{array}$ \\
\hline
\end{tabular}


Table S17 Optimization procedure for case G

\begin{tabular}{|c|c|c|c|c|}
\hline Step & $\begin{array}{l}\text { Objective } \\
\text { Function }\end{array}$ & Decision Variables $(\beta)$ & Fixed Variables & Constraints \\
\hline 1 & $\max _{\beta}\{$ Profit $\}$ & $\begin{array}{c}Q_{\mathrm{I}} \\
Q_{D} \\
Q_{X} \\
\text { Tol/MeOH } \\
\text { Water/HC } \\
\text { Space time }\end{array}$ & $\begin{array}{c}\text { Stream } 1 \\
\text { Recovery OX } \\
t_{s} \\
N_{\mathrm{I}} \mathrm{Col} \\
N_{\mathrm{II}} \mathrm{Col} \\
N_{\mathrm{IV}}{ }^{\mathrm{Col}}\end{array}$ & $\begin{array}{c}\sum_{k} N_{k}^{C o l}=24 \\
\mathrm{PX}_{\text {Stream } 11} \geq 99.7 \mathrm{wt} \% \\
\Delta \mathrm{P}_{\mathrm{SMB}} \leq 685 \mathrm{kPa} \\
\Delta \mathrm{P} / \text { bed }_{\mathrm{SMB}} \leq 35 \mathrm{kPa} \\
\text { Recovery } \\
\text { MeOH loss } \leq 97 \% \\
\text { MeO } 040\end{array}$ \\
\hline 2 & $\max _{\beta}\{$ Profit $\}$ & $\begin{array}{c}Q_{\mathrm{I}} \\
Q_{D} \\
Q_{X} \\
N_{\mathrm{I}} \mathrm{Col} \\
N_{\mathrm{II}} \mathrm{Col} \\
N_{\mathrm{IV}}{ }^{C o l} \\
\text { Tol/MeOH } \\
\text { Water/HC } \\
\text { Space time }\end{array}$ & $\begin{array}{c}\text { Stream } 1 \\
\text { Recovery OX } \\
t_{s}\end{array}$ & $\begin{array}{c}\sum_{k} N_{k}^{C o l}=24 \\
\mathrm{PX}_{\text {Stream } 11} \geq 99.7 \mathrm{wt} \% \\
\Delta \mathrm{P}_{\mathrm{SMB}} \leq 685 \mathrm{kPa} \\
\Delta \mathrm{P} / \text { bed }_{\mathrm{SMB}} \leq 35 \mathrm{kPa} \\
\text { Recovery } \\
\mathrm{MeOH} \text { loss } \leq 40 \%\end{array}$ \\
\hline 3 & $\max _{\beta}\{$ Profit $\}$ & $\begin{array}{c}Q_{\mathrm{I}} \\
Q_{D} \\
Q_{X} \\
t_{s} \\
\text { Tol/MeOH } \\
\text { Water/HC } \\
\text { Space time }\end{array}$ & $\begin{array}{c}\text { Stream } 1 \\
\text { Recovery OX } \\
N_{\mathrm{I}} \mathrm{Col} \\
N_{\mathrm{II}} \mathrm{Col} \\
N_{\mathrm{IV}}{ }^{C o l}\end{array}$ & $\begin{array}{c}\sum_{k} N_{k}^{C o l}=24 \\
\mathrm{PX}_{\text {Stream } 11} \geq 99.7 \mathrm{wt} \% \\
\Delta \mathrm{P}_{\mathrm{SMB}} \leq 685 \mathrm{kPa} \\
\Delta \mathrm{P} / \text { bed }_{\mathrm{SMB}} \leq 35 \mathrm{kPa} \\
\text { Recovery }_{\mathrm{SMB}} \geq 97 \% \\
\text { MeOH loss } \leq 40 \%\end{array}$ \\
\hline 4 & $\max _{\beta}\{$ Profit $\}$ & $\begin{array}{c}Q_{\mathrm{I}} \\
Q_{D} \\
Q_{X} \\
t_{s} \\
\text { Tol/MeOH } \\
\text { Water/HC } \\
\text { Space time } \\
\end{array}$ & $\begin{array}{c}\text { Stream } 1 \\
\text { Recovery OX } \\
N_{\mathrm{I}} \mathrm{Col} \\
N_{\mathrm{II}} \mathrm{Col} \\
N_{\mathrm{IV}}{ }^{\mathrm{Col}}\end{array}$ & $\begin{array}{c}\sum_{k} N_{k}^{C o l}=24 \\
\mathrm{PX}_{\text {Stream } 11} \geq 99.7 \mathrm{wt} \% \\
\Delta \mathrm{P}_{\mathrm{SMB}} \leq 685 \mathrm{kPa} \\
\Delta \mathrm{P} / \text { bed }_{\mathrm{SMB}} \leq 35 \mathrm{kPa} \\
\text { Recovery } \\
\text { MeOH loss } \leq 40 \%\end{array}$ \\
\hline 5 & $\max _{\beta}\{$ Profit $\}$ & $\begin{array}{c}Q_{\mathrm{I}} \\
Q_{D} \\
Q_{X} \\
t_{s} \\
N_{\mathrm{I}} \mathrm{Col} \\
N_{\mathrm{II}} \mathrm{Col} \\
N_{\mathrm{IV}}{ }^{C o l} \\
\text { Tol/MeOH } \\
\text { Water/HC } \\
\text { Space time }\end{array}$ & $\begin{array}{c}\text { Stream } 1 \\
\text { Recovery OX }\end{array}$ & $\begin{array}{c}\sum_{k} N_{k}^{C o l}=24 \\
\mathrm{PX}_{\text {Stream } 11} \geq 99.7 \mathrm{wt} \% \\
\Delta \mathrm{P}_{\mathrm{SMB}} \leq 685 \mathrm{kPa} \\
\Delta \mathrm{P} / \text { bed }_{\mathrm{SMB}} \leq 35 \mathrm{kPa} \\
\text { Recovery } \\
\text { MeOH loss } \leq 40 \%\end{array}$ \\
\hline
\end{tabular}




\section{S7. Case A results}

Table S18 Optimization of the current aromatics complex (A)

\begin{tabular}{lccccc} 
& Step 1 & Step 2 & Step 3 & Step 4 & Step 5 \\
\hline Profit $\left(\$ / t_{\text {Reformate }}\right)$ & 950.6 & 952.7 & 957.4 & 957.2 & 957.4 \\
$\Delta$ Revenue $\times 10^{-3}(\$ / \text { year) })^{\mathrm{a}}$ & -1135 & -511 & 899 & 840 & 901 \\
Operation Cost $(\$ / \mathrm{h}):$ & & & & & \\
Aromatics Extraction & 353.6 & 353.6 & 353.6 & 353.6 & 353.6 \\
Benzene Column & 142.8 & 142.8 & 142.8 & 142.8 & 142.8 \\
Xylene Splitter & 294.0 & 294.8 & 295.9 & 295.9 & 296.0 \\
Extract Column & 160.4 & 162.8 & 115.4 & 116.8 & 115.2 \\
Raffinate Column & 609.2 & 543.3 & 443.1 & 447.7 & 443.2 \\
Isomerization & 367.8 & 362.0 & 353.6 & 354.0 & 353.5 \\
$o-X y l e n e ~ C o l u m n$ & 61.4 & 61.4 & 61.4 & 61.4 & 61.4 \\
SMB: & & & & & \\
Configuration & $5-9-7-3$ & $8-6-4-6$ & $8-6-4-6$ & $5-9-7-3$ & $10-5-3-6$ \\
Switching time $(\mathrm{s})$ & 66 & 66 & 180 & 180 & 180 \\
Productivity $\left(\mathrm{kg} / \mathrm{hm}{ }^{3}\right)$ & 46.27 & 46.27 & 46.22 & 46.22 & 46.22 \\
Desorbent consumption $(\mathrm{kg} / \mathrm{kg})$ & 8.09 & 6.31 & 2.00 & 2.17 & 1.99 \\
Recovery $(\%)$ & 97.00 & 98.17 & 99.94 & 99.84 & 99.95 \\
Total $\Delta \mathrm{P}(\mathrm{kPa})$ & 233 & 227 & 67 & 70 & 67 \\
Maximum $\Delta \mathrm{P} / \mathrm{bed}(\mathrm{kPa})$ & 10.8 & 10.7 & 3.7 & 3.7 & 3.7 \\
\hline
\end{tabular}

a Compared to the base case (A0) assuming 330 days of operation per year. 
Table S19 Mass balance for the current aromatics complex base case (A0)

\begin{tabular}{lcccccccccc}
\hline Stream & 1 & 2 & 3 & 4 & 5 & 6 & 7 & 8 & 9 & 10 \\
\hline Flow (t/h) & 38.10 & 20.60 & 17.50 & 62.78 & 55.52 & 39.15 & 68.66 & 7.26 & 4.89 & 15.71 \\
Ben (wt\%) & 12.86 & 23.78 & 0.00 & 0.00 & 0.00 & 0.00 & 0.00 & 0.00 & 99.88 & 0.08 \\
Tol (wt\%) & 41.21 & 76.22 & 0.00 & 0.00 & 0.00 & 0.00 & 0.00 & 0.00 & 0.12 & 99.92 \\
ECH (wt\%) & 0.00 & 0.00 & 0.00 & 18.05 & 20.41 & 0.00 & 16.51 & 0.00 & 0.00 & 0.00 \\
EB (wt\%) & 3.20 & 0.00 & 6.97 & 6.32 & 7.15 & 0.00 & 5.78 & 0.00 & 0.00 & 0.00 \\
PX (wt\%) & 6.56 & 0.00 & 14.28 & 16.39 & 18.53 & 26.32 & 0.02 & 0.00 & 0.00 & 0.00 \\
MX (wt\%) & 13.88 & 0.00 & 30.22 & 35.03 & 39.60 & 0.00 & 32.01 & 0.09 & 0.00 & 0.00 \\
OX (wt\%) & 6.29 & 0.00 & 13.69 & 14.41 & 14.31 & 0.030 & 11.58 & 15.15 & 0.00 & 0.00 \\
TMBen (wt\%) & 16.00 & 0.00 & 34.84 & 9.71 & 0.00 & 0.00 & 0.00 & 83.98 & 0.00 & 0.00 \\
PDEB (wt\%) & 0.00 & 0.00 & 0.00 & 0.09 & 0.00 & 73.65 & 34.10 & 0.78 & 0.00 & 0.00 \\
\hline Stream & 11 & 12 & 13 & 14 & 15 & 16 & 17 & 18 & 19 & \\
\hline Flow (t/h) & 10.31 & 1.10 & 6.16 & 28.84 & 23.38 & 52.22 & 45.29 & 45.29 & 20.60 & \\
Ben (wt\%) & 0.00 & 0.00 & 0.00 & 0.00 & 0.00 & 0.00 & 0.00 & 0.00 & 23.78 & \\
Tol (wt\%) & 0.00 & 0.00 & 0.00 & 0.00 & 0.00 & 0.00 & 0.00 & 0.00 & 76.22 & \\
ECH (wt\%) & 0.02 & 0.00 & 0.00 & 0.00 & 0.00 & 0.00 & 25.03 & 25.03 & 0.00 & \\
EB (wt\%) & 0.00 & 0.00 & 0.00 & 0.00 & 0.00 & 0.00 & 8.76 & 6.07 & 0.00 & \\
PX (wt\%) & 99.71 & 0.00 & 0.00 & 0.08 & 0.00 & 0.04 & 0.02 & 17.20 & 0.00 & \\
MX (wt\%) & 0.01 & 0.59 & 0.00 & 0.00 & 0.00 & 0.00 & 48.55 & 36.89 & 0.00 & \\
OX (wt\%) & 0.13 & 99.29 & 0.09 & 0.00 & 0.08 & 0.04 & 17.52 & 14.69 & 0.00 & \\
TMBen (wt\%) & 0.00 & 0.12 & 98.99 & 0.00 & 0.00 & 0.00 & 0.00 & 0.00 & 0.00 & \\
PDEB (wt\%) & 0.13 & 0.00 & 0.92 & 99.92 & 99.92 & 99.92 & 0.12 & 0.12 & 0.00 & \\
\hline
\end{tabular}


Table S20 Mass balance for the current aromatics complex after step 1 (A1)

\begin{tabular}{|c|c|c|c|c|c|c|c|c|c|c|}
\hline Stream & 1 & 2 & 3 & 4 & 5 & 6 & 7 & 8 & 9 & 10 \\
\hline Flow $(\mathrm{t} / \mathrm{h})$ & 38.10 & 20.60 & 17.50 & 64.57 & 57.31 & 32.17 & 108.53 & 7.26 & 4.89 & 15.71 \\
\hline $\operatorname{Ben}\left(w t^{0} \%\right)$ & 12.86 & 23.78 & 0.00 & 0.00 & 0.00 & 0.00 & 0.00 & 0.00 & 99.88 & 0.08 \\
\hline Tol (wt\%) & 41.21 & 76.22 & 0.00 & 0.00 & 0.00 & 0.00 & 0.00 & 0.00 & 0.12 & 99.92 \\
\hline $\mathrm{ECH}\left(\mathrm{wt}^{\circ} \%\right)$ & 0.00 & 0.00 & 0.00 & 18.25 & 20.56 & 0.00 & 10.85 & 0.00 & 0.00 & 0.00 \\
\hline $\mathrm{EB}(\mathrm{wt} \%)$ & 3.20 & 0.00 & 6.97 & 6.31 & 7.11 & 0.00 & 3.76 & 0.00 & 0.00 & 0.00 \\
\hline $\mathrm{PX}(w \mathrm{t} \%)$ & 6.56 & 0.00 & 14.28 & 16.41 & 18.49 & 32.00 & 0.29 & 0.00 & 0.00 & 0.00 \\
\hline $\mathrm{MX}(\mathrm{wt} \%)$ & 13.88 & 0.00 & 30.22 & 35.08 & 39.51 & 0.00 & 20.86 & 0.09 & 0.00 & 0.00 \\
\hline $\mathrm{OX}(\mathrm{wt} \%)$ & 6.29 & 0.00 & 13.69 & 14.42 & 14.32 & 0.05 & 7.59 & 15.15 & 0.00 & 0.00 \\
\hline TMBen (wt\%) & 16.00 & 0.00 & 34.84 & 9.44 & 0.00 & 0.00 & 0.00 & 83.95 & 0.00 & 0.00 \\
\hline PDEB $(w t \%)$ & 0.00 & 0.00 & 0.00 & 0.09 & 0.00 & 67.94 & 56.64 & 0.81 & 0.00 & 0.00 \\
\hline Stream & 11 & 12 & 13 & 14 & 15 & 16 & 17 & 18 & 19 & \\
\hline Flow $(\mathrm{t} / \mathrm{h})$ & 10.31 & 1.10 & 6.16 & 21.86 & 61.46 & 83.33 & 47.07 & 47.07 & 20.60 & \\
\hline $\operatorname{Ben}\left(w t^{0} \%\right)$ & 0.00 & 0.00 & 0.00 & 0.00 & 0.00 & 0.00 & 0.00 & 0.00 & 23.78 & \\
\hline Tol (wt\%) & 0.00 & 0.00 & 0.00 & 0.00 & 0.00 & 0.00 & 0.00 & 0.00 & 76.22 & \\
\hline $\mathrm{ECH}(\mathrm{wt} \%)$ & 0.00 & 0.00 & 0.00 & 0.00 & 0.00 & 0.00 & 25.03 & 25.03 & 0.00 & \\
\hline $\mathrm{EB}(\mathrm{wt} \%)$ & 0.00 & 0.00 & 0.00 & 0.00 & 0.00 & 0.00 & 8.66 & 6.07 & 0.00 & \\
\hline $\mathrm{PX}(\mathrm{wt} \%)$ & 99.70 & 0.00 & 0.00 & 0.08 & 0.00 & 0.02 & 0.68 & 17.20 & 0.00 & \\
\hline $\mathrm{MX}(\mathrm{wt} \%)$ & 0.00 & 0.59 & 0.00 & 0.00 & 0.00 & 0.00 & 48.11 & 36.89 & 0.00 & \\
\hline $\mathrm{OX}(\mathrm{wt} \%)$ & 0.17 & 99.29 & 0.09 & 0.00 & 0.08 & 0.06 & 17.40 & 14.68 & 0.00 & \\
\hline TMBen $(w t \%)$ & 0.00 & 0.11 & 98.96 & 0.00 & 0.00 & 0.00 & 0.00 & 0.00 & 0.00 & \\
\hline PDEB (wt $\%)$ & 0.13 & 0.00 & 0.95 & 99.92 & 99.92 & 99.92 & 0.12 & 0.12 & 0.00 & \\
\hline
\end{tabular}


Table S21 Mass balance for the current aromatics complex after step 2 (A2)

\begin{tabular}{lcccccccccc}
\hline Stream & 1 & 2 & 3 & 4 & 5 & 6 & 7 & 8 & 9 & 10 \\
\hline Flow (t/h) & 38.10 & 20.60 & 17.50 & 63.82 & 56.57 & 32.95 & 88.65 & 7.26 & 4.89 & 15.71 \\
Ben (wt\%) & 12.86 & 23.78 & 0.00 & 0.00 & 0.00 & 0.00 & 0.00 & 0.00 & 99.88 & 0.08 \\
Tol (wt\%) & 41.21 & 76.22 & 0.00 & 0.00 & 0.00 & 0.00 & 0.00 & 0.00 & 0.12 & 99.92 \\
ECH (wt\%) & 0.00 & 0.00 & 0.00 & 18.17 & 20.50 & 0.00 & 13.08 & 0.00 & 0.00 & 0.00 \\
EB (wt\%) & 3.20 & 0.00 & 6.97 & 6.32 & 7.13 & 0.00 & 4.55 & 0.00 & 0.00 & 0.00 \\
PX (wt\%) & 6.56 & 0.00 & 14.28 & 16.40 & 18.51 & 31.24 & 0.22 & 0.00 & 0.00 & 0.00 \\
MX (wt\%) & 13.88 & 0.00 & 30.22 & 35.06 & 39.55 & 0.00 & 25.23 & 0.09 & 0.00 & 0.00 \\
OX (wt\%) & 6.29 & 0.00 & 13.69 & 14.41 & 14.32 & 0.05 & 9.16 & 15.15 & 0.00 & 0.00 \\
TMBen (wt\%) & 16.00 & 0.00 & 34.84 & 9.55 & 0.00 & 0.00 & 0.00 & 83.96 & 0.00 & 0.00 \\
PDEB (wt\%) & 0.00 & 0.00 & 0.00 & 0.09 & 0.00 & 68.70 & 47.77 & 0.80 & 0.00 & 0.00 \\
\hline Stream & 11 & 12 & 13 & 14 & 15 & 16 & 17 & 18 & 19 & \\
\hline Flow (t/h) & 10.31 & 1.10 & 6.16 & 22.65 & 42.32 & 64.96 & 46.33 & 46.33 & 20.60 & \\
Ben (wt\%) & 0.00 & 0.00 & 0.00 & 0.00 & 0.00 & 0.00 & 0.00 & 0.00 & 23.78 & \\
Tol (wt\%) & 0.00 & 0.00 & 0.00 & 0.00 & 0.00 & 0.00 & 0.00 & 0.00 & 76.22 & \\
ECH (wt\%) & 0.01 & 0.00 & 0.00 & 0.00 & 0.00 & 0.00 & 25.03 & 25.03 & 0.00 & \\
EB (wt\%) & 0.00 & 0.00 & 0.00 & 0.00 & 0.00 & 0.00 & 8.70 & 6.07 & 0.00 & \\
PX (wt\%) & 99.70 & 0.00 & 0.00 & 0.08 & 0.00 & 0.03 & 0.41 & 17.20 & 0.00 & \\
MX (wt\%) & 0.01 & 0.59 & 0.00 & 0.00 & 0.00 & 0.00 & 48.28 & 36.89 & 0.00 & \\
OX (wt\%) & 0.16 & 99.29 & 0.09 & 0.00 & 0.08 & 0.05 & 17.45 & 14.68 & 0.00 & \\
TMBen (wt\%) & 0.00 & 0.11 & 98.97 & 0.00 & 0.00 & 0.00 & 0.00 & 0.00 & 0.00 & \\
PDEB (wt\%) & 0.13 & 0.00 & 0.94 & 99.92 & 99.92 & 99.92 & 0.12 & 0.12 & 0.00 & \\
\hline
\end{tabular}


Table S22 Mass balance for the current aromatics complex after step 3 (A3)

\begin{tabular}{lcccccccccc}
\hline Stream & 1 & 2 & 3 & 4 & 5 & 6 & 7 & 8 & 9 & 10 \\
\hline Flow (t/h) & 38.10 & 20.60 & 17.50 & 62.75 & 55.49 & 17.51 & 58.54 & 7.26 & 4.89 & 15.71 \\
Ben (wt\%) & 12.86 & 23.78 & 0.00 & 0.00 & 0.00 & 0.00 & 0.00 & 0.00 & 99.88 & 0.08 \\
Tol (wt\%) & 41.21 & 76.22 & 0.00 & 0.00 & 0.00 & 0.00 & 0.00 & 0.00 & 0.12 & 99.92 \\
ECH (wt\%) & 0.00 & 0.00 & 0.00 & 18.05 & 20.41 & 0.06 & 19.33 & 0.00 & 0.00 & 0.00 \\
EB (wt\%) & 3.20 & 0.00 & 6.97 & 6.32 & 7.15 & 0.00 & 6.78 & 0.00 & 0.00 & 0.00 \\
PX (wt\%) & 6.56 & 0.00 & 14.28 & 16.39 & 18.53 & 58.72 & 0.01 & 0.00 & 0.00 & 0.00 \\
MX (wt\%) & 13.88 & 0.00 & 30.22 & 35.03 & 39.60 & 0.00 & 37.54 & 0.09 & 0.00 & 0.00 \\
OX (wt\%) & 6.29 & 0.00 & 13.69 & 14.41 & 14.31 & 0.04 & 13.57 & 15.15 & 0.00 & 0.00 \\
TMBen (wt\%) & 16.00 & 0.00 & 34.84 & 9.71 & 0.00 & 0.00 & 0.00 & 83.98 & 0.00 & 0.00 \\
PDEB (wt\%) & 0.00 & 0.00 & 0.00 & 0.09 & 0.00 & 41.18 & 22.77 & 0.78 & 0.00 & 0.00 \\
\hline Stream & 11 & 12 & 13 & 14 & 15 & 16 & 17 & 18 & 19 & \\
\hline Flow (t/h) & 10.31 & 1.10 & 6.16 & 7.20 & 13.28 & 20.49 & 45.25 & 45.25 & 20.60 & \\
Ben (wt\%) & 0.00 & 0.00 & 0.00 & 0.00 & 0.00 & 0.00 & 0.00 & 0.00 & 23.78 & \\
Tol (wt\%) & 0.00 & 0.00 & 0.00 & 0.00 & 0.00 & 0.00 & 0.00 & 0.00 & 76.22 & \\
ECH (wt\%) & 0.09 & 0.00 & 0.00 & 0.00 & 0.00 & 0.00 & 25.03 & 25.03 & 0.00 & \\
EB (wt\%) & 0.00 & 0.00 & 0.00 & 0.00 & 0.00 & 0.00 & 8.76 & 6.07 & 0.00 & \\
PX (wt\%) & 99.70 & 0.00 & 0.00 & 0.08 & 0.00 & 0.03 & 0.01 & 17.20 & 0.00 & \\
MX (wt\%) & 0.00 & 0.59 & 0.00 & 0.00 & 0.00 & 0.00 & 48.56 & 36.89 & 0.00 & \\
OX (wt\%) & 0.07 & 99.29 & 0.09 & 0.00 & 0.08 & 0.05 & 17.52 & 14.68 & 0.00 & \\
TMBen (wt\%) & 0.00 & 0.11 & 99.00 & 0.00 & 0.00 & 0.00 & 0.00 & 0.00 & 0.00 & \\
PDEB (wt\%) & 0.13 & 0.00 & 0.92 & 99.92 & 99.92 & 99.92 & 0.12 & 0.12 & 0.00 & \\
\hline
\end{tabular}


Table S23 Mass balance for the current aromatics complex after step 4 (A4)

\begin{tabular}{lcccccccccc}
\hline Stream & 1 & 2 & 3 & 4 & 5 & 6 & 7 & 8 & 9 & 10 \\
\hline Flow (t/h) & 38.10 & 20.60 & 17.50 & 62.81 & 55.55 & 17.98 & 59.87 & 7.26 & 4.89 & 15.71 \\
Ben (wt\%) & 12.86 & 23.78 & 0.00 & 0.00 & 0.00 & 0.00 & 0.00 & 0.00 & 99.88 & 0.08 \\
Tol (wt\%) & 41.21 & 76.22 & 0.00 & 0.00 & 0.00 & 0.00 & 0.00 & 0.00 & 0.12 & 99.92 \\
ECH (wt\%) & 0.00 & 0.00 & 0.00 & 18.06 & 20.42 & 0.04 & 18.93 & 0.00 & 0.00 & 0.00 \\
EB (wt\%) & 3.20 & 0.00 & 6.97 & 6.32 & 7.15 & 0.00 & 6.63 & 0.00 & 0.00 & 0.00 \\
PX (wt\%) & 6.56 & 0.00 & 14.28 & 16.39 & 18.53 & 57.20 & 0.03 & 0.00 & 0.00 & 0.00 \\
MX (wt\%) & 13.88 & 0.00 & 30.22 & 35.03 & 39.60 & 0.01 & 36.74 & 0.09 & 0.00 & 0.00 \\
OX (wt\%) & 6.29 & 0.00 & 13.69 & 14.41 & 14.31 & 0.05 & 13.29 & 15.15 & 0.00 & 0.00 \\
TMBen (wt\%) & 16.00 & 0.00 & 34.84 & 9.70 & 0.00 & 0.00 & 0.00 & 83.98 & 0.00 & 0.00 \\
PDEB (wt\%) & 0.00 & 0.00 & 0.00 & 0.09 & 0.00 & 42.70 & 24.39 & 0.78 & 0.00 & 0.00 \\
\hline Stream & 11 & 12 & 13 & 14 & 15 & 16 & 17 & 18 & 19 & \\
\hline Flow (t/h) & 10.31 & 1.10 & 6.16 & 7.67 & 14.55 & 22.22 & 45.31 & 45.31 & 20.60 & \\
Ben (wt\%) & 0.00 & 0.00 & 0.00 & 0.00 & 0.00 & 0.00 & 0.00 & 0.00 & 23.78 & \\
Tol (wt\%) & 0.00 & 0.00 & 0.00 & 0.00 & 0.00 & 0.00 & 0.00 & 0.00 & 76.22 & \\
ECH (wt\%) & 0.07 & 0.00 & 0.00 & 0.00 & 0.00 & 0.00 & 25.03 & 25.03 & 0.00 & \\
EB (wt\%) & 0.00 & 0.00 & 0.00 & 0.00 & 0.00 & 0.00 & 8.76 & 6.07 & 0.00 & \\
PX (wt\%) & 99.70 & 0.00 & 0.00 & 0.08 & 0.00 & 0.03 & 0.04 & 17.20 & 0.00 & \\
MX (wt\%) & 0.02 & 0.59 & 0.00 & 0.00 & 0.00 & 0.00 & 48.54 & 36.89 & 0.00 & \\
OX (wt\%) & 0.08 & 99.29 & 0.09 & 0.00 & 0.08 & 0.05 & 17.52 & 14.68 & 0.00 & \\
TMBen (wt\%) & 0.00 & 0.11 & 98.99 & 0.00 & 0.00 & 0.00 & 0.00 & 0.00 & 0.00 & \\
PDEB (wt\%) & 0.13 & 0.00 & 0.92 & 99.92 & 99.92 & 99.92 & 0.12 & 0.12 & 0.00 & \\
\hline
\end{tabular}


Table S24 Mass balance for the current aromatics complex after step 5 (A5)

\begin{tabular}{|c|c|c|c|c|c|c|c|c|c|c|}
\hline Stream & 1 & 2 & 3 & 4 & 5 & 6 & 7 & 8 & 9 & 10 \\
\hline Flow (t/h) & 38.10 & 20.60 & 17.50 & 62.74 & 55.48 & 17.43 & 58.57 & 7.26 & 4.89 & 15.71 \\
\hline Ben (wt\%) & 12.86 & 23.78 & 0.00 & 0.00 & 0.00 & 0.00 & 0.00 & 0.00 & 99.88 & 0.08 \\
\hline Tol (wt\%) & 41.21 & 76.22 & 0.00 & 0.00 & 0.00 & 0.00 & 0.00 & 0.00 & 0.12 & 99.92 \\
\hline $\mathrm{ECH}(\mathrm{wt} \%)$ & 0.00 & 0.00 & 0.00 & 18.05 & 20.41 & 0.05 & 19.32 & 0.00 & 0.00 & 0.00 \\
\hline EB (wt\%) & 3.20 & 0.00 & 6.97 & 6.32 & 7.15 & 0.00 & 6.77 & 0.00 & 0.00 & 0.00 \\
\hline PX (wt\%) & 6.56 & 0.00 & 14.28 & 16.39 & 18.53 & 58.99 & 0.01 & 0.00 & 0.00 & 0.00 \\
\hline $\mathrm{MX}(\mathrm{wt} \%)$ & 13.88 & 0.00 & 30.22 & 35.03 & 39.60 & 0.00 & 37.51 & 0.09 & 0.00 & 0.00 \\
\hline OX (wt\%) & 6.29 & 0.00 & 13.69 & 14.41 & 14.31 & 0.04 & 13.56 & 15.15 & 0.00 & 0.00 \\
\hline TMBen (wt\%) & 16.00 & 0.00 & 34.84 & 9.71 & 0.00 & 0.00 & 0.00 & 83.98 & 0.00 & 0.00 \\
\hline PDEB (wt \%) & 0.00 & 0.00 & 0.00 & 0.09 & 0.00 & 40.91 & 22.83 & 0.78 & 0.00 & 0.00 \\
\hline Stream & 11 & 12 & 13 & 14 & 15 & 16 & 17 & 18 & 19 & \\
\hline Flow (t/h) & 10.31 & 1.10 & 6.16 & 7.12 & 13.32 & 20.45 & 45.24 & 45.24 & 20.60 & \\
\hline Ben $(w t \%)$ & 0.00 & 0.000 & 0.000 & 0.00 & 0.00 & 0.00 & 0.00 & 0.00 & 23.78 & \\
\hline Tol (wt\%) & 0.00 & 0.000 & 0.000 & 0.00 & 0.00 & 0.00 & 0.00 & 0.00 & 76.22 & \\
\hline $\mathrm{ECH}(\mathrm{wt} \%)$ & 0.09 & 0.000 & 0.000 & 0.00 & 0.00 & 0.00 & 25.03 & 25.03 & 0.00 & \\
\hline EB (wt\%) & 0.01 & 0.000 & 0.000 & 0.00 & 0.00 & 0.00 & 8.76 & 6.07 & 0.00 & \\
\hline PX (wt\%) & 99.70 & 0.000 & 0.000 & 0.08 & 0.00 & 0.03 & 0.01 & 17.20 & 0.00 & \\
\hline MX (wt\%) & 0.00 & 0.593 & 0.000 & 0.00 & 0.00 & 0.00 & 48.56 & 36.89 & 0.00 & \\
\hline $\mathrm{OX}(\mathrm{wt} \%)$ & 0.07 & 99.294 & 0.088 & 0.00 & 0.08 & 0.05 & 17.52 & 14.68 & 0.00 & \\
\hline TMBen $(w t \%)$ & 0.00 & 0.113 & 98.996 & 0.00 & 0.00 & 0.00 & 0.00 & 0.00 & 0.00 & \\
\hline PDEB (wt\%) & 0.13 & 0.000 & 0.916 & 99.92 & 99.92 & 99.92 & 0.12 & 0.12 & 0.00 & \\
\hline
\end{tabular}




\section{S8. Case B results}

Table S25 Optimization of the modified aromatics complex with separation of naphthenes within the isomerization unit (B)

\begin{tabular}{|c|c|c|c|c|c|}
\hline & Step 1 & Step 2 & Step 3 & Step 4 & Step 5 \\
\hline Profit ( $\left.\$ / t_{\text {Reformate }}\right)$ & 899.4 & 902.4 & 908.3 & 908.0 & 908.3 \\
\hline$\Delta$ Revenue $\times 10^{-3}(\$ / \text { year })^{\mathrm{a}}$ & -16593 & -15690 & -13915 & -13989 & -13905 \\
\hline \multicolumn{6}{|l|}{ Operation Cost $(\$ / \mathrm{h})$ : } \\
\hline Aromatics Extraction & 353.6 & 353.6 & 353.6 & 353.6 & 353.6 \\
\hline Benzene Column & 142.8 & 142.8 & 142.8 & 142.8 & 142.8 \\
\hline Xylene Splitter & 271.2 & 272.1 & 272.8 & 272.8 & 272.8 \\
\hline Extract Column & 162.0 & 172.8 & 116.1 & 118.1 & 115.2 \\
\hline Raffinate Column & 526.8 & 458.9 & 355.9 & 361.2 & 356.2 \\
\hline Isomerization & 2168.6 & 2125.6 & 2089.7 & 2090.5 & 2089.2 \\
\hline$o$-Xylene Column & 61.4 & 61.4 & 61.4 & 61.4 & 61.4 \\
\hline \multicolumn{6}{|l|}{ SMB: } \\
\hline Configuration & $5-9-7-3$ & $7-7-4-6$ & $7-7-4-6$ & $5-9-7-3$ & $10-5-3-6$ \\
\hline Switching time (s) & 66 & 66 & 180 & 180 & 180 \\
\hline Productivity $\left(\mathrm{kg} / \mathrm{hm}^{3}\right)$ & 46.27 & 46.28 & 46.22 & 46.22 & 46.21 \\
\hline Desorbent consumption $(\mathrm{kg} / \mathrm{kg})$ & 8.04 & 6.58 & 1.93 & 2.14 & 1.91 \\
\hline Recovery (\%) & 97.17 & 98.67 & 99.95 & 99.92 & 99.96 \\
\hline Total $\Delta \mathrm{P}(\mathrm{kPa})$ & 231 & 226 & 66 & 68 & 67 \\
\hline Maximum $\Delta \mathrm{P} /$ bed $(\mathrm{kPa})$ & 10.6 & 10.4 & 3.4 & 3.4 & 3.4 \\
\hline
\end{tabular}

a Compared to the base case (A0) assuming 330 days of operation per year. 
Table S26 Mass balance for the modified aromatics complex with separation of naphthenes within the isomerization unit after step 1 (B1)

\begin{tabular}{|c|c|c|c|c|c|c|c|c|c|c|}
\hline Stream & 1 & 2 & 3 & 4 & 5 & 6 & 7 & 8 & 9 & 10 \\
\hline Flow $(\mathrm{t} / \mathrm{h})$ & 38.10 & 20.60 & 17.50 & 52.70 & 45.45 & 32.68 & 95.61 & 7.25 & 4.89 & 15.71 \\
\hline Ben (wt\%) & 12.86 & 23.78 & 0.00 & 0.00 & 0.00 & 0.00 & 0.00 & 0.00 & 99.88 & 0.08 \\
\hline Tol (wt\%) & 41.21 & 76.22 & 0.00 & 0.00 & 0.00 & 0.00 & 0.00 & 0.00 & 0.12 & 99.92 \\
\hline $\mathrm{ECH}(\mathrm{wt} \%)$ & 0.00 & 0.00 & 0.00 & 0.00 & 0.00 & 0.00 & 0.00 & 0.00 & 0.00 & 0.00 \\
\hline $\mathrm{EB}(w \mathrm{t} \%)$ & 3.20 & 0.00 & 6.97 & 7.72 & 8.96 & 0.00 & 4.26 & 0.00 & 0.00 & 0.00 \\
\hline $\mathrm{PX}(\mathrm{wt} \%)$ & 6.56 & 0.00 & 14.28 & 20.07 & 23.27 & 31.50 & 0.31 & 0.00 & 0.00 & 0.00 \\
\hline MX (wt\%) & 13.88 & 0.00 & 30.22 & 42.91 & 49.74 & 0.00 & 23.64 & 0.09 & 0.00 & 0.00 \\
\hline $\mathrm{OX}(\mathrm{wt} \%)$ & 6.29 & 0.00 & 13.69 & 17.64 & 18.04 & 0.05 & 8.61 & 15.18 & 0.00 & 0.00 \\
\hline TMBen $(w t \%)$ & 16.00 & 0.00 & 34.84 & 11.57 & 0.00 & 0.00 & 0.00 & 84.12 & 0.00 & 0.00 \\
\hline PDEB (wt\%) & 0.00 & 0.00 & 0.00 & 0.08 & 0.00 & 68.45 & 63.18 & 0.61 & 0.00 & 0.00 \\
\hline Stream & 11 & 12 & 13 & 14 & 15 & 16 & 17 & 18 & 19 & \\
\hline Flow $(\mathrm{t} / \mathrm{h})$ & 10.31 & 1.10 & 6.14 & 22.38 & 60.41 & 82.79 & 35.20 & 35.20 & 20.60 & \\
\hline Ben (wt\%) & 0.00 & 0.00 & 0.00 & 0.00 & 0.00 & 0.00 & 0.00 & 0.00 & 23.78 & \\
\hline Tol (wt\%) & 0.00 & 0.00 & 0.00 & 0.00 & 0.00 & 0.00 & 0.00 & 0.00 & 76.22 & \\
\hline $\mathrm{ECH}(\mathrm{wt} \%)$ & 0.00 & 0.00 & 0.00 & 0.00 & 0.00 & 0.00 & 0.00 & 0.00 & 0.00 & \\
\hline $\mathrm{EB}(\mathrm{wt} \%)$ & 0.00 & 0.00 & 0.00 & 0.00 & 0.00 & 0.00 & 11.56 & 8.10 & 0.00 & \\
\hline $\mathrm{PX}(w t \%)$ & 99.70 & 0.00 & 0.00 & 0.08 & 0.00 & 0.02 & 0.85 & 22.95 & 0.00 & \\
\hline $\mathrm{MX}(\mathrm{wt} \%)$ & 0.00 & 0.59 & 0.00 & 0.00 & 0.00 & 0.00 & 64.22 & 49.22 & 0.00 & \\
\hline $\mathrm{OX}(\mathrm{wt} \%)$ & 0.17 & 99.29 & 0.09 & 0.00 & 0.08 & 0.06 & 23.24 & 19.61 & 0.00 & \\
\hline TMBen $(w t \%)$ & 0.00 & 0.11 & 99.19 & 0.00 & 0.00 & 0.00 & 0.00 & 0.00 & 0.00 & \\
\hline PDEB (wt\%) & 0.13 & 0.00 & 0.72 & 99.92 & 99.92 & 99.92 & 0.13 & 0.13 & 0.00 & \\
\hline
\end{tabular}


Table S27 Mass balance for the modified aromatics complex with separation of naphthenes within the isomerization unit after step 2 (B2)

\begin{tabular}{lcccccccccc}
\hline Stream & 1 & 2 & 3 & 4 & 5 & 6 & 7 & 8 & 9 & 10 \\
\hline Flow (t/h) & 38.10 & 20.60 & 17.50 & 52.00 & 44.75 & 36.25 & 76.29 & 7.25 & 4.89 & 15.71 \\
Ben (wt\%) & 12.86 & 23.78 & 0.00 & 0.00 & 0.00 & 0.00 & 0.00 & 0.00 & 99.88 & 0.08 \\
Tol (wt\%) & 0.00 & 0.00 & 0.00 & 0.00 & 0.00 & 0.00 & 0.00 & 0.00 & 0.00 & 0.00 \\
ECH (wt\%) & 41.21 & 76.22 & 0.00 & 0.00 & 0.00 & 0.00 & 0.00 & 0.00 & 0.12 & 99.92 \\
EB (wt\%) & 0.00 & 0.00 & 0.00 & 0.00 & 0.00 & 0.00 & 0.00 & 0.00 & 0.00 & 0.00 \\
PX (wt\%) & 3.20 & 0.00 & 6.97 & 7.72 & 8.97 & 0.00 & 5.26 & 0.00 & 0.00 & 0.00 \\
MX (wt\%) & 6.56 & 0.00 & 14.28 & 20.03 & 23.28 & 28.41 & 0.18 & 0.00 & 0.00 & 0.00 \\
OX (wt\%) & 13.88 & 0.00 & 30.22 & 42.83 & 49.74 & 0.00 & 29.18 & 0.09 & 0.00 & 0.00 \\
TMBen (wt\%) & 6.29 & 0.00 & 13.69 & 17.62 & 18.01 & 0.05 & 10.59 & 15.18 & 0.00 & 0.00 \\
PDEB (wt\%) & 16.00 & 0.00 & 34.84 & 11.72 & 0.00 & 0.00 & 0.00 & 84.13 & 0.00 & 0.00 \\
\hline Stream & 11 & 12 & 13 & 14 & 15 & 16 & 17 & 18 & 19 & \\
\hline Flow (t/h) & 10.31 & 1.10 & 6.14 & 25.94 & 41.78 & 67.72 & 34.50 & 34.50 & 20.60 & \\
Ben (wt\%) & 0.00 & 0.00 & 0.00 & 0.00 & 0.00 & 0.00 & 0.00 & 0.00 & 23.78 & \\
Tol (wt\%) & 0.00 & 0.00 & 0.00 & 0.00 & 0.00 & 0.00 & 0.00 & 0.00 & 0.00 & \\
ECH (wt\%) & 0.00 & 0.00 & 0.00 & 0.00 & 0.00 & 0.00 & 0.00 & 0.00 & 76.22 & \\
EB (wt\%) & 0.00 & 0.00 & 0.00 & 0.00 & 0.00 & 0.00 & 0.00 & 0.00 & 0.00 & \\
PX (wt\%) & 0.00 & 0.00 & 0.00 & 0.00 & 0.00 & 0.00 & 11.63 & 8.10 & 0.00 & \\
MX (wt\%) & 99.70 & 0.00 & 0.00 & 0.08 & 0.00 & 0.03 & 0.40 & 22.95 & 0.00 & \\
OX (wt\%) & 0.01 & 0.59 & 0.00 & 0.00 & 0.00 & 0.00 & 64.52 & 49.22 & 0.00 & \\
TMBen (wt\%) & 0.16 & 99.29 & 0.09 & 0.00 & 0.08 & 0.05 & 23.31 & 19.61 & 0.00 & \\
PDEB (wt\%) & 0.00 & 0.11 & 99.20 & 0.00 & 0.00 & 0.00 & 0.00 & 0.00 & 0.00 & \\
\hline & & & & & & & & & & \\
& & & & & & & & \\
& & & & & & \\
& & &
\end{tabular}


Table S28 Mass balance for the modified aromatics complex with separation of naphthenes within the isomerization unit after step 3 (B3)

\begin{tabular}{|c|c|c|c|c|c|c|c|c|c|c|}
\hline Stream & 1 & 2 & 3 & 4 & 5 & 6 & 7 & 8 & 9 & 10 \\
\hline Flow (t/h) & 38.10 & 20.60 & 17.50 & 51.42 & 44.17 & 17.74 & 46.27 & 7.24 & 4.89 & 15.71 \\
\hline Ben (wt\%) & 12.86 & 23.78 & 0.00 & 0.00 & 0.00 & 0.00 & 0.00 & 0.00 & 99.88 & 0.08 \\
\hline Tol (wt\%) & 41.21 & 76.22 & 0.00 & 0.00 & 0.00 & 0.00 & 0.00 & 0.00 & 0.12 & 99.92 \\
\hline $\mathrm{ECH}(\mathrm{wt} \%)$ & 0.00 & 0.00 & 0.00 & 0.00 & 0.00 & 0.00 & 0.00 & 0.00 & 0.00 & 0.00 \\
\hline $\mathrm{EB}(w \mathrm{t} \%)$ & 3.20 & 0.00 & 6.97 & 7.72 & 8.98 & 0.00 & 8.57 & 0.00 & 0.00 & 0.00 \\
\hline $\mathrm{PX}(\mathrm{wt} \%)$ & 6.56 & 0.00 & 14.28 & 20.00 & 23.28 & 57.95 & 0.01 & 0.00 & 0.00 & 0.00 \\
\hline MX (wt\%) & 13.88 & 0.00 & 30.22 & 42.75 & 49.75 & 0.05 & 47.47 & 0.09 & 0.00 & 0.00 \\
\hline $\mathrm{OX}(\mathrm{wt} \%)$ & 6.29 & 0.00 & 13.69 & 17.59 & 17.99 & 0.05 & 17.18 & 15.18 & 0.00 & 0.00 \\
\hline TMBen $(w t \%)$ & 16.00 & 0.00 & 34.84 & 11.85 & 0.00 & 0.00 & 0.00 & 84.14 & 0.00 & 0.00 \\
\hline PDEB (wt\%) & 0.00 & 0.00 & 0.00 & 0.08 & 0.00 & 41.95 & 26.77 & 0.59 & 0.00 & 0.00 \\
\hline Stream & 11 & 12 & 13 & 14 & 15 & 16 & 17 & 18 & 19 & \\
\hline Flow $(\mathrm{t} / \mathrm{h})$ & 10.31 & 1.10 & 6.14 & 7.44 & 12.35 & 19.79 & 33.92 & 33.92 & 20.60 & \\
\hline Ben (wt\%) & 0.00 & 0.00 & 0.00 & 0.00 & 0.00 & 0.00 & 0.00 & 0.00 & 23.78 & \\
\hline Tol (wt\%) & 0.00 & 0.00 & 0.00 & 0.00 & 0.00 & 0.00 & 0.00 & 0.00 & 76.22 & \\
\hline $\mathrm{ECH}(\mathrm{wt} \%)$ & 0.00 & 0.00 & 0.00 & 0.00 & 0.00 & 0.00 & 0.00 & 0.00 & 0.00 & \\
\hline $\mathrm{EB}(w \mathrm{t} \%)$ & 0.00 & 0.00 & 0.00 & 0.00 & 0.00 & 0.00 & 11.69 & 8.10 & 0.00 & \\
\hline $\mathrm{PX}(w t \%)$ & 99.70 & 0.00 & 0.00 & 0.08 & 0.00 & 0.03 & 0.02 & 22.95 & 0.00 & \\
\hline $\mathrm{MX}(\mathrm{wt} \%)$ & 0.09 & 0.59 & 0.00 & 0.00 & 0.00 & 0.00 & 64.76 & 49.22 & 0.00 & \\
\hline $\mathrm{OX}(\mathrm{wt} \%)$ & 0.08 & 99.29 & 0.09 & 0.00 & 0.08 & 0.05 & 23.40 & 19.61 & 0.00 & \\
\hline TMBen $(w t \%)$ & 0.00 & 0.11 & 99.21 & 0.00 & 0.00 & 0.00 & 0.00 & 0.00 & 0.00 & \\
\hline PDEB (wt\%) & 0.13 & 0.00 & 0.70 & 99.92 & 99.92 & 99.92 & 0.13 & 0.13 & 0.00 & \\
\hline
\end{tabular}


Table S29 Mass balance for the modified aromatics complex with separation of naphthenes within the isomerization unit after step 4 (B4)

\begin{tabular}{|c|c|c|c|c|c|c|c|c|c|c|}
\hline Stream & 1 & 2 & 3 & 4 & 5 & 6 & 7 & 8 & 9 & 10 \\
\hline Flow (t/h) & 38.10 & 20.60 & 17.50 & 51.43 & 44.19 & 18.37 & 47.83 & 7.24 & 4.89 & 15.71 \\
\hline Ben (wt\%) & 12.86 & 23.78 & 0.00 & 0.00 & 0.00 & 0.00 & 0.00 & 0.00 & 99.88 & 0.08 \\
\hline Tol (wt\%) & 41.21 & 76.22 & 0.00 & 0.00 & 0.00 & 0.00 & 0.00 & 0.00 & 0.12 & 99.92 \\
\hline $\mathrm{ECH}(\mathrm{wt} \%)$ & 0.00 & 0.00 & 0.00 & 0.00 & 0.00 & 0.00 & 0.00 & 0.00 & 0.00 & 0.00 \\
\hline $\mathrm{EB}(\mathrm{wt} \%)$ & 3.20 & 0.00 & 6.97 & 7.72 & 8.98 & 0.00 & 8.30 & 0.00 & 0.00 & 0.00 \\
\hline $\mathrm{PX}(\mathrm{wt} \%)$ & 6.56 & 0.00 & 14.28 & 20.00 & 23.28 & 55.97 & 0.02 & 0.00 & 0.00 & 0.00 \\
\hline MX (wt\%) & 13.88 & 0.00 & 30.22 & 42.75 & 49.75 & 0.04 & 45.94 & 0.09 & 0.00 & 0.00 \\
\hline OX (wt\%) & 6.29 & 0.00 & 13.69 & 17.59 & 17.99 & 0.05 & 16.62 & 15.18 & 0.00 & 0.00 \\
\hline TMBen (wt\%) & 16.00 & 0.00 & 34.84 & 11.85 & 0.00 & 0.00 & 0.00 & 84.14 & 0.00 & 0.00 \\
\hline PDEB (wt\%) & 0.00 & 0.00 & 0.00 & 0.08 & 0.00 & 43.93 & 29.12 & 0.59 & 0.00 & 0.00 \\
\hline Stream & 11 & 12 & 13 & 14 & 15 & 16 & 17 & 18 & 19 & \\
\hline Flow $(\mathrm{t} / \mathrm{h})$ & 10.31 & 1.10 & 6.14 & 8.06 & 13.90 & 21.96 & 33.93 & 33.93 & 20.60 & \\
\hline Ben (wt\%) & 0.00 & 0.00 & 0.00 & 0.00 & 0.00 & 0.00 & 0.00 & 0.00 & 23.78 & \\
\hline Tol (wt\%) & 0.00 & 0.00 & 0.00 & 0.00 & 0.00 & 0.00 & 0.00 & 0.00 & 76.22 & \\
\hline $\mathrm{ECH}(\mathrm{wt} \%)$ & 0.00 & 0.00 & 0.00 & 0.00 & 0.00 & 0.00 & 0.00 & 0.00 & 0.00 & \\
\hline $\mathrm{EB}(\mathrm{wt} \%)$ & 0.00 & 0.00 & 0.00 & 0.00 & 0.00 & 0.00 & 11.69 & 8.10 & 0.00 & \\
\hline $\mathrm{PX}(\mathrm{wt} \%)$ & 99.70 & 0.00 & 0.00 & 0.08 & 0.00 & 0.03 & 0.03 & 22.95 & 0.00 & \\
\hline MX (wt\%) & 0.08 & 0.59 & 0.00 & 0.00 & 0.00 & 0.00 & 64.76 & 49.22 & 0.00 & \\
\hline $\mathrm{OX}(\mathrm{wt} \%)$ & 0.09 & 99.29 & 0.09 & 0.00 & 0.08 & 0.05 & 23.40 & 19.61 & 0.00 & \\
\hline TMBen $(w t \%)$ & 0.00 & 0.11 & 99.21 & 0.00 & 0.00 & 0.00 & 0.00 & 0.00 & 0.00 & \\
\hline PDEB (wt\%) & 0.13 & 0.00 & 0.70 & 99.92 & 99.92 & 99.92 & 0.13 & 0.13 & 0.00 & \\
\hline
\end{tabular}


Table S30 Mass balance for the modified aromatics complex with separation of naphthenes within the isomerization unit after step 5 (B5)

\begin{tabular}{|c|c|c|c|c|c|c|c|c|c|c|}
\hline Stream & 1 & 2 & 3 & 4 & 5 & 6 & 7 & 8 & 9 & 10 \\
\hline Flow $(\mathrm{t} / \mathrm{h})$ & 38.10 & 20.60 & 17.50 & 51.41 & 44.16 & 17.44 & 46.38 & 7.24 & 4.89 & 15.71 \\
\hline Ben (wt\%) & 12.86 & 23.78 & 0.00 & 0.00 & 0.00 & 0.00 & 0.00 & 0.00 & 99.88 & 0.08 \\
\hline Tol (wt\%) & 41.21 & 76.22 & 0.00 & 0.00 & 0.00 & 0.00 & 0.00 & 0.00 & 0.12 & 99.92 \\
\hline $\mathrm{ECH}(\mathrm{wt} \%)$ & 0.00 & 0.00 & 0.00 & 0.00 & 0.00 & 0.00 & 0.00 & 0.00 & 0.00 & 0.00 \\
\hline $\mathrm{EB}(w \mathrm{t} \%)$ & 3.20 & 0.00 & 6.97 & 7.72 & 8.98 & 0.00 & 8.55 & 0.00 & 0.00 & 0.00 \\
\hline $\mathrm{PX}(\mathrm{wt} \%)$ & 6.56 & 0.00 & 14.28 & 20.00 & 23.28 & 58.96 & 0.01 & 0.00 & 0.00 & 0.00 \\
\hline MX (wt\%) & 13.88 & 0.00 & 30.22 & 42.75 & 49.75 & 0.05 & 47.35 & 0.09 & 0.00 & 0.00 \\
\hline $\mathrm{OX}(\mathrm{wt} \%)$ & 6.29 & 0.00 & 13.69 & 17.59 & 17.99 & 0.05 & 17.13 & 15.18 & 0.00 & 0.00 \\
\hline TMBen $(w t \%)$ & 16.00 & 0.00 & 34.84 & 11.86 & 0.00 & 0.00 & 0.00 & 84.14 & 0.00 & 0.00 \\
\hline PDEB (wt\%) & 0.00 & 0.00 & 0.00 & 0.08 & 0.00 & 40.94 & 26.96 & 0.59 & 0.00 & 0.00 \\
\hline Stream & 11 & 12 & 13 & 14 & 15 & 16 & 17 & 18 & 19 & \\
\hline Flow $(\mathrm{t} / \mathrm{h})$ & 10.31 & 1.10 & 6.14 & 7.13 & 12.47 & 19.60 & 33.91 & 33.91 & 20.60 & \\
\hline Ben (wt\%) & 0.00 & 0.00 & 0.00 & 0.00 & 0.00 & 0.00 & 0.00 & 0.00 & 23.78 & \\
\hline Tol (wt\%) & 0.00 & 0.00 & 0.00 & 0.00 & 0.00 & 0.00 & 0.00 & 0.00 & 76.22 & \\
\hline $\mathrm{ECH}(\mathrm{wt} \%)$ & 0.00 & 0.00 & 0.00 & 0.00 & 0.00 & 0.00 & 0.00 & 0.00 & 0.00 & \\
\hline $\mathrm{EB}(w \mathrm{t} \%)$ & 0.00 & 0.00 & 0.00 & 0.00 & 0.00 & 0.00 & 11.69 & 8.10 & 0.00 & \\
\hline $\mathrm{PX}(w t \%)$ & 99.70 & 0.00 & 0.00 & 0.08 & 0.00 & 0.03 & 0.01 & 22.95 & 0.00 & \\
\hline $\mathrm{MX}(\mathrm{wt} \%)$ & 0.09 & 0.59 & 0.00 & 0.00 & 0.00 & 0.00 & 64.76 & 49.22 & 0.00 & \\
\hline $\mathrm{OX}(\mathrm{wt} \%)$ & 0.08 & 99.29 & 0.09 & 0.00 & 0.08 & 0.05 & 23.40 & 19.61 & 0.00 & \\
\hline TMBen $(w t \%)$ & 0.00 & 0.11 & 99.21 & 0.00 & 0.00 & 0.00 & 0.00 & 0.00 & 0.00 & \\
\hline PDEB (wt\%) & 0.13 & 0.00 & 0.70 & 99.92 & 99.92 & 99.92 & 0.13 & 0.13 & 0.00 & \\
\hline
\end{tabular}


S9. Case $\mathrm{C}$ results

Table S31 Optimization of the modified aromatics complex with crystallization at $218 \mathrm{~K}$ (C)

\begin{tabular}{lccccc} 
& Step 1 & Step 2 & Step 3 & Step 4 & Step 5 \\
\hline Profit $\left(\$ / t_{\text {Reformate }}\right)$ & 949.5 & 950.5 & 953.9 & 953.8 & 953.9 \\
$\Delta$ Revenue $\times 10^{-3}(\$ / \text { year })^{\mathrm{a}}$ & -1472 & -1160 & -146 & -184 & -146 \\
Operation Cost $(\$ / \mathrm{h}):$ & & & & & \\
Aromatics Extraction & 353.6 & 353.6 & 353.6 & 353.6 & 353.6 \\
Benzene Column & 142.8 & 142.8 & 142.8 & 142.8 & 142.8 \\
Xylene Splitter & 294.0 & 294.8 & 295.6 & 295.5 & 295.5 \\
Extract Column & 201.4 & 180.3 & 119.9 & 121.5 & 119.9 \\
Raffinate Column & 487.3 & 480.9 & 435.4 & 437.4 & 435.5 \\
Isomerization & 368.9 & 363.0 & 357.5 & 358.1 & 357.5 \\
$o-X y l e n e ~ C o l u m n$ & 61.5 & 61.5 & 61.5 & 61.5 & 61.5 \\
Crystallization & 99.9 & 98.2 & 97.4 & 97.4 & 97.4 \\
SMB: & & & & & \\
Configuration & $5-9-7-3$ & $10-5-3-6$ & $10-5-3-6$ & $5-9-7-3$ & $10-4-4-6$ \\
Switching time $(\mathrm{s})$ & 66 & 66 & 180 & 180 & 180 \\
Productivity $\left(\mathrm{kg} / \mathrm{hm}{ }^{3}\right)$ & 46.97 & 46.77 & 46.60 & 46.62 & 46.60 \\
Desorbent consumption $(\mathrm{kg} / \mathrm{kg})$ & 5.46 & 4.83 & 1.76 & 1.85 & 1.76 \\
Recovery $(\%)$ & 98.18 & 99.00 & 99.96 & 99.86 & 99.96 \\
Total $\Delta \mathrm{P}(\mathrm{kPa})$ & 227 & 227 & 67 & 70 & 68 \\
Maximum $\Delta \mathrm{P} / \mathrm{bed}(\mathrm{kPa})$ & 10.3 & 10.4 & 3.7 & 3.7 & 3.7 \\
\hline
\end{tabular}

a Compared to the base case (A0) assuming 330 days of operation per year. 
Table S32 Mass balance for the modified aromatics complex with crystallization at $218 \mathrm{~K}$ after step 1 (C1)

\begin{tabular}{lccccccccccc}
\hline Stream & 1 & 2 & 3 & 4 & 5 & 6 & 7 & 8 & 9 & 10 & 11 \\
\hline Flow (t/h) & 38.10 & 20.60 & 17.50 & 64.71 & 57.44 & 43.74 & 70.83 & 7.27 & 4.89 & 15.71 & 10.30 \\
Ben (wt\%) & 12.86 & 23.78 & 0.00 & 0.00 & 0.00 & 0.00 & 0.00 & 0.00 & 99.88 & 0.08 & 0.00 \\
Tol (wt\%) & 41.21 & 76.22 & 0.00 & 0.00 & 0.00 & 0.00 & 0.00 & 0.00 & 0.12 & 99.92 & 0.00 \\
ECH (wt\%) & 0.00 & 0.00 & 0.00 & 18.26 & 20.57 & 0.82 & 16.18 & 0.00 & 0.00 & 0.00 & 0.00 \\
EB (wt\%) & 3.20 & 0.00 & 6.97 & 6.31 & 7.11 & 0.12 & 5.69 & 0.00 & 0.00 & 0.00 & 0.00 \\
PX (wt\%) & 6.56 & 0.00 & 14.28 & 16.41 & 18.49 & 23.89 & 0.27 & 0.00 & 0.00 & 0.00 & 100.00 \\
MX (wt\%) & 13.88 & 0.00 & 30.22 & 35.07 & 39.50 & 0.84 & 31.51 & 0.09 & 0.00 & 0.00 & 0.00 \\
OX (wt\%) & 6.29 & 0.00 & 13.69 & 14.41 & 14.32 & 0.29 & 11.46 & 15.12 & 0.00 & 0.00 & 0.00 \\
TMBen (wt\%) & 16.00 & 0.00 & 34.84 & 9.42 & 0.00 & 0.00 & 0.00 & 83.80 & 0.00 & 0.00 & 0.00 \\
PDEB (wt\%) & 0.00 & 0.00 & 0.00 & 0.11 & 0.00 & 74.04 & 34.88 & 0.99 & 0.00 & 0.00 & 0.00 \\
\hline Stream & 12 & 13 & 14 & 15 & 16 & 17 & 18 & 19 & 20 & 21 & 22 \\
\hline Flow (t/h) & 1.10 & 6.17 & 32.40 & 24.67 & 57.07 & 47.21 & 47.21 & 20.60 & 11.35 & 46.16 & 1.05 \\
Ben (wt\%) & 0.00 & 0.00 & 0.00 & 0.00 & 0.00 & 0.00 & 0.00 & 23.78 & 0.00 & 0.00 & 0.00 \\
Tol (wt\%) & 0.00 & 0.00 & 0.00 & 0.00 & 0.00 & 0.00 & 0.00 & 76.22 & 0.00 & 0.00 & 0.00 \\
ECH (wt\%) & 0.00 & 0.00 & 0.00 & 0.00 & 0.00 & 25.03 & 25.03 & 0.00 & 3.17 & 24.82 & 34.23 \\
EB (wt\%) & 0.00 & 0.00 & 0.00 & 0.00 & 0.00 & 8.65 & 6.07 & 0.00 & 0.45 & 8.74 & 4.89 \\
PX (wt\%) & 0.00 & 0.00 & 0.08 & 0.00 & 0.04 & 0.68 & 17.20 & 0.00 & 91.88 & 0.42 & 12.29 \\
MX (wt\%) & 0.59 & 0.00 & 0.00 & 0.00 & 0.00 & 48.06 & 36.87 & 0.00 & 3.24 & 48.36 & 35.03 \\
OX (wt\%) & 99.29 & 0.09 & 0.00 & 0.08 & 0.03 & 17.43 & 14.68 & 0.00 & 1.13 & 17.54 & 12.20 \\
TMBen (wt\%) & 0.11 & 98.75 & 0.00 & 0.00 & 0.00 & 0.00 & 0.00 & 0.00 & 0.00 & 0.00 & 0.00 \\
PDEB (wt\%) & 0.00 & 1.16 & 99.92 & 99.92 & 99.92 & 0.15 & 0.15 & 0.00 & 0.13 & 0.12 & 1.36 \\
\hline & & & & & & & & & & &
\end{tabular}


Table S33 Mass balance for the modified aromatics complex with crystallization at $218 \mathrm{~K}$ after step 2 (C2)

\begin{tabular}{lccccccccccc}
\hline Stream & 1 & 2 & 3 & 4 & 5 & 6 & 7 & 8 & 9 & 10 & 11 \\
\hline Flow (t/h) & 38.10 & 20.60 & 17.50 & 63.96 & 56.69 & 37.47 & 69.57 & 7.27 & 4.89 & 15.71 & 10.30 \\
Ben (wt\%) & 12.86 & 23.78 & 0.00 & 0.00 & 0.00 & 0.00 & 0.00 & 0.00 & 99.88 & 0.08 & 0.00 \\
Tol (wt\%) & 41.21 & 76.22 & 0.00 & 0.00 & 0.00 & 0.00 & 0.00 & 0.00 & 0.12 & 99.92 & 0.00 \\
ECH (wt\%) & 0.00 & 0.00 & 0.00 & 18.18 & 20.52 & 0.86 & 16.26 & 0.00 & 0.00 & 0.00 & 0.00 \\
EB (wt\%) & 3.20 & 0.00 & 6.97 & 6.31 & 7.12 & 0.09 & 5.76 & 0.00 & 0.00 & 0.00 & 0.00 \\
PX (wt\%) & 6.56 & 0.00 & 14.28 & 16.40 & 18.50 & 27.77 & 0.15 & 0.00 & 0.00 & 0.00 & 100.00 \\
MX (wt\%) & 13.88 & 0.00 & 30.22 & 35.05 & 39.54 & 0.57 & 31.91 & 0.09 & 0.00 & 0.00 & 0.00 \\
OX (wt\%) & 6.29 & 0.00 & 13.69 & 14.41 & 14.32 & 0.20 & 11.59 & 15.12 & 0.00 & 0.00 & 0.00 \\
TMBen (wt\%) & 16.00 & 0.00 & 34.84 & 9.53 & 0.00 & 0.00 & 0.00 & 83.81 & 0.00 & 0.00 & 0.00 \\
PDEB (wt\%) & 0.00 & 0.00 & 0.00 & 0.11 & 0.00 & 70.52 & 34.33 & 0.98 & 0.00 & 0.00 & 0.00 \\
\hline Stream & 12 & 13 & 14 & 15 & 16 & 17 & 18 & 19 & 20 & 21 & 22 \\
\hline Flow (t/h) & 1.10 & 6.17 & 26.43 & 23.85 & 50.28 & 46.47 & 46.47 & 20.60 & 11.04 & 45.72 & 0.74 \\
Ben (wt\%) & 0.00 & 0.00 & 0.00 & 0.00 & 0.00 & 0.00 & 0.00 & 23.78 & 0.00 & 0.00 & 0.00 \\
Tol (wt\%) & 0.00 & 0.00 & 0.00 & 0.00 & 0.00 & 0.00 & 0.00 & 76.22 & 0.00 & 0.00 & 0.00 \\
ECH (wt\%) & 0.00 & 0.00 & 0.00 & 0.00 & 0.00 & 25.03 & 25.03 & 0.00 & 2.91 & 24.74 & 43.12 \\
EB (wt\%) & 0.00 & 0.00 & 0.00 & 0.00 & 0.00 & 8.69 & 6.07 & 0.00 & 0.29 & 8.76 & 4.31 \\
PX (wt\%) & 0.00 & 0.00 & 0.08 & 0.00 & 0.04 & 0.42 & 17.20 & 0.00 & 94.06 & 0.23 & 12.00 \\
MX (wt\%) & 0.59 & 0.00 & 0.00 & 0.00 & 0.00 & 48.24 & 36.87 & 0.00 & 1.93 & 48.56 & 28.59 \\
OX (wt\%) & 99.29 & 0.09 & 0.00 & 0.08 & 0.04 & 17.47 & 14.68 & 0.00 & 0.68 & 17.59 & 10.11 \\
TMBen (wt\%) & 0.11 & 98.76 & 0.00 & 0.00 & 0.00 & 0.00 & 0.00 & 0.00 & 0.00 & 0.00 & 0.00 \\
PDEB (wt\%) & 0.00 & 1.15 & 99.92 & 99.92 & 99.92 & 0.15 & 0.15 & 0.00 & 0.13 & 0.12 & 1.87 \\
\hline & & & & & & & & & & &
\end{tabular}


Table S34 Mass balance for the modified aromatics complex with crystallization at $218 \mathrm{~K}$ after step 3 (C3)

\begin{tabular}{lccccccccccc}
\hline Stream & 1 & 2 & 3 & 4 & 5 & 6 & 7 & 8 & 9 & 10 & 11 \\
\hline Flow (t/h) & 38.10 & 20.60 & 17.50 & 63.25 & 55.98 & 18.02 & 56.24 & 7.27 & 4.89 & 15.71 & 10.30 \\
Ben (wt\%) & 12.86 & 23.78 & 0.00 & 0.00 & 0.00 & 0.00 & 0.00 & 0.00 & 99.88 & 0.08 & 0.00 \\
Tol (wt\%) & 41.21 & 76.22 & 0.00 & 0.00 & 0.00 & 0.00 & 0.00 & 0.00 & 0.12 & 99.92 & 0.00 \\
ECH (wt\%) & 0.00 & 0.00 & 0.00 & 18.11 & 20.46 & 2.17 & 19.67 & 0.00 & 0.00 & 0.00 & 0.00 \\
EB (wt\%) & 3.20 & 0.00 & 6.97 & 6.32 & 7.14 & 0.01 & 7.10 & 0.00 & 0.00 & 0.00 & 0.00 \\
PX (wt\%) & 6.56 & 0.00 & 14.28 & 16.39 & 18.52 & 57.54 & 0.01 & 0.00 & 0.00 & 0.00 & 100.00 \\
MX (wt\%) & 13.88 & 0.00 & 30.22 & 35.03 & 39.57 & 0.63 & 39.19 & 0.09 & 0.00 & 0.00 & 0.00 \\
OX (wt\%) & 6.29 & 0.00 & 13.69 & 14.41 & 14.31 & 0.14 & 14.22 & 15.13 & 0.00 & 0.00 & 0.00 \\
TMBen (wt\%) & 16.00 & 0.00 & 34.84 & 9.64 & 0.00 & 0.00 & 0.00 & 83.82 & 0.00 & 0.00 & 0.00 \\
PDEB (wt\%) & 0.00 & 0.00 & 0.00 & 0.11 & 0.00 & 39.50 & 19.82 & 0.96 & 0.00 & 0.00 & 0.00 \\
\hline Stream & 12 & 13 & 14 & 15 & 16 & 17 & 18 & 19 & 20 & 21 & 22 \\
\hline Flow (t/h) & 1.10 & 6.17 & 7.11 & 11.10 & 18.21 & 45.76 & 45.76 & 20.60 & 10.91 & 45.14 & 0.61 \\
Ben (wt\%) & 0.00 & 0.00 & 0.00 & 0.00 & 0.00 & 0.00 & 0.00 & 23.78 & 0.00 & 0.00 & 0.00 \\
Tol (wt\%) & 0.00 & 0.00 & 0.00 & 0.00 & 0.00 & 0.00 & 0.00 & 76.22 & 0.00 & 0.00 & 0.00 \\
ECH (wt\%) & 0.00 & 0.00 & 0.00 & 0.00 & 0.00 & 25.03 & 25.03 & 0.00 & 3.58 & 24.50 & 63.78 \\
EB (wt\%) & 0.00 & 0.00 & 0.00 & 0.00 & 0.00 & 8.73 & 6.07 & 0.00 & 0.02 & 8.85 & 0.39 \\
PX (wt\%) & 0.00 & 0.00 & 0.08 & 0.00 & 0.03 & 0.16 & 17.20 & 0.00 & 95.00 & 0.01 & 10.96 \\
MX (wt\%) & 0.59 & 0.00 & 0.00 & 0.00 & 0.00 & 48.42 & 36.87 & 0.00 & 1.05 & 48.82 & 18.60 \\
OX (wt\%) & 99.29 & 0.09 & 0.00 & 0.08 & 0.05 & 17.51 & 14.68 & 0.00 & 0.23 & 17.70 & 4.03 \\
TMBen (wt\%) & 0.11 & 98.78 & 0.00 & 0.00 & 0.00 & 0.00 & 0.00 & 0.00 & 0.00 & 0.00 & 0.00 \\
PDEB (wt\%) & 0.00 & 1.14 & 99.92 & 99.92 & 99.92 & 0.15 & 0.15 & 0.00 & 0.13 & 0.12 & 2.24 \\
\hline & & & & & & & & & & &
\end{tabular}


Table S35 Mass balance for the modified aromatics complex with crystallization at $218 \mathrm{~K}$ after step 4 (C4)

\begin{tabular}{lccccccccccc}
\hline Stream & 1 & 2 & 3 & 4 & 5 & 6 & 7 & 8 & 9 & 10 & 11 \\
\hline Flow (t/h) & 38.10 & 20.60 & 17.50 & 63.34 & 56.06 & 18.49 & 56.75 & 7.27 & 4.89 & 15.71 & 10.30 \\
Ben (wt\%) & 12.86 & 23.78 & 0.00 & 0.00 & 0.00 & 0.00 & 0.00 & 0.00 & 99.88 & 0.08 & 0.00 \\
Tol (wt\%) & 41.21 & 76.22 & 0.00 & 0.00 & 0.00 & 0.00 & 0.00 & 0.00 & 0.12 & 99.92 & 0.00 \\
ECH (wt\%) & 0.00 & 0.00 & 0.00 & 18.12 & 20.46 & 1.79 & 19.63 & 0.00 & 0.00 & 0.00 & 0.00 \\
EB (wt\%) & 3.20 & 0.00 & 6.97 & 6.32 & 7.14 & 0.03 & 7.04 & 0.00 & 0.00 & 0.00 & 0.00 \\
PX (wt\%) & 6.56 & 0.00 & 14.28 & 16.39 & 18.52 & 56.10 & 0.03 & 0.00 & 0.00 & 0.00 & 100.00 \\
MX (wt\%) & 13.88 & 0.00 & 30.22 & 35.04 & 39.57 & 0.83 & 38.82 & 0.09 & 0.00 & 0.00 & 0.00 \\
OX (wt\%) & 6.29 & 0.00 & 13.69 & 14.41 & 14.31 & 0.23 & 14.08 & 15.13 & 0.00 & 0.00 & 0.00 \\
TMBen (wt\%) & 16.00 & 0.00 & 34.84 & 9.62 & 0.00 & 0.00 & 0.00 & 83.82 & 0.00 & 0.00 & 0.00 \\
PDEB (wt\%) & 0.00 & 0.00 & 0.00 & 0.11 & 0.00 & 41.02 & 20.40 & 0.96 & 0.00 & 0.00 & 0.00 \\
\hline Stream & 12 & 13 & 14 & 15 & 16 & 17 & 18 & 19 & 20 & 21 & 22 \\
\hline Flow (t/h) & 1.10 & 6.17 & 7.58 & 11.53 & 19.11 & 45.84 & 45.84 & 20.60 & 10.91 & 45.22 & 0.62 \\
Ben (wt\%) & 0.00 & 0.00 & 0.00 & 0.00 & 0.00 & 0.00 & 0.00 & 23.78 & 0.00 & 0.00 & 0.00 \\
Tol (wt\%) & 0.00 & 0.00 & 0.00 & 0.00 & 0.00 & 0.00 & 0.00 & 76.22 & 0.00 & 0.00 & 0.00 \\
ECH (wt\%) & 0.00 & 0.00 & 0.00 & 0.00 & 0.00 & 25.03 & 25.03 & 0.00 & 3.04 & 24.64 & 53.78 \\
EB (wt\%) & 0.00 & 0.00 & 0.00 & 0.00 & 0.00 & 8.73 & 6.07 & 0.00 & 0.05 & 8.84 & 0.83 \\
PX (wt\%) & 0.00 & 0.00 & 0.08 & 0.00 & 0.03 & 0.19 & 17.20 & 0.00 & 95.00 & 0.03 & 11.53 \\
MX (wt\%) & 0.59 & 0.00 & 0.00 & 0.00 & 0.00 & 48.39 & 36.87 & 0.00 & 1.40 & 48.72 & 24.85 \\
OX (wt\%) & 99.29 & 0.09 & 0.00 & 0.08 & 0.05 & 17.51 & 14.68 & 0.00 & 0.38 & 17.65 & 6.77 \\
TMBen (wt\%) & 0.11 & 98.77 & 0.00 & 0.00 & 0.00 & 0.00 & 0.00 & 0.00 & 0.00 & 0.00 & 0.00 \\
PDEB (wt\%) & 0.00 & 1.14 & 99.92 & 99.92 & 99.92 & 0.15 & 0.15 & 0.00 & 0.13 & 0.12 & 2.23 \\
\hline & & & & & & & & & & &
\end{tabular}


Table S36 Mass balance for the modified aromatics complex with crystallization at $218 \mathrm{~K}$ after step 5 (C5)

\begin{tabular}{lccccccccccc}
\hline Stream & 1 & 2 & 3 & 4 & 5 & 6 & 7 & 8 & 9 & 10 & 11 \\
\hline Flow (t/h) & 38.10 & 20.60 & 17.50 & 63.25 & 55.98 & 18.00 & 56.26 & 7.27 & 4.89 & 15.71 & 10.30 \\
Ben (wt\%) & 12.86 & 23.78 & 0.00 & 0.00 & 0.00 & 0.00 & 0.00 & 0.00 & 99.88 & 0.08 & 0.00 \\
Tol (wt\%) & 41.21 & 76.22 & 0.00 & 0.00 & 0.00 & 0.00 & 0.00 & 0.00 & 0.12 & 99.92 & 0.00 \\
ECH (wt\%) & 0.00 & 0.00 & 0.00 & 18.11 & 20.46 & 2.17 & 19.66 & 0.00 & 0.00 & 0.00 & 0.00 \\
EB (wt\%) & 3.20 & 0.00 & 6.97 & 6.32 & 7.14 & 0.03 & 7.09 & 0.00 & 0.00 & 0.00 & 0.00 \\
PX (wt\%) & 6.56 & 0.00 & 14.28 & 16.39 & 18.52 & 57.61 & 0.01 & 0.00 & 0.00 & 0.00 & 100.00 \\
MX (wt\%) & 13.88 & 0.00 & 30.22 & 35.03 & 39.57 & 0.63 & 39.17 & 0.09 & 0.00 & 0.00 & 0.00 \\
OX (wt\%) & 6.29 & 0.00 & 13.69 & 14.41 & 14.31 & 0.14 & 14.21 & 15.13 & 0.00 & 0.00 & 0.00 \\
TMBen (wt\%) & 16.00 & 0.00 & 34.84 & 9.64 & 0.00 & 0.00 & 0.00 & 83.82 & 0.00 & 0.00 & 0.00 \\
PDEB (wt\%) & 0.00 & 0.00 & 0.00 & 0.11 & 0.00 & 39.42 & 19.85 & 0.96 & 0.00 & 0.00 & 0.00 \\
\hline Stream & 12 & 13 & 14 & 15 & 16 & 17 & 18 & 19 & 20 & 21 & 22 \\
\hline Flow (t/h) & 1.10 & 6.17 & 7.09 & 11.12 & 18.21 & 45.76 & 45.76 & 20.60 & 10.91 & 45.14 & 0.62 \\
Ben (wt\%) & 0.00 & 0.00 & 0.00 & 0.00 & 0.00 & 0.00 & 0.00 & 23.78 & 0.00 & 0.00 & 0.00 \\
Tol (wt\%) & 0.00 & 0.00 & 0.00 & 0.00 & 0.00 & 0.00 & 0.00 & 76.22 & 0.00 & 0.00 & 0.00 \\
ECH (wt\%) & 0.00 & 0.00 & 0.00 & 0.00 & 0.00 & 25.03 & 25.03 & 0.00 & 3.58 & 24.51 & 63.28 \\
EB (wt\%) & 0.00 & 0.00 & 0.00 & 0.00 & 0.00 & 8.73 & 6.07 & 0.00 & 0.05 & 8.84 & 0.92 \\
PX (wt\%) & 0.00 & 0.00 & 0.08 & 0.00 & 0.03 & 0.16 & 17.20 & 0.00 & 94.97 & 0.01 & 11.00 \\
MX (wt\%) & 0.59 & 0.00 & 0.00 & 0.00 & 0.00 & 48.41 & 36.87 & 0.00 & 1.05 & 48.82 & 18.51 \\
OX (wt\%) & 99.29 & 0.09 & 0.00 & 0.08 & 0.05 & 17.51 & 14.68 & 0.00 & 0.23 & 17.70 & 4.07 \\
TMBen (wt\%) & 0.11 & 98.78 & 0.00 & 0.00 & 0.00 & 0.00 & 0.00 & 0.00 & 0.00 & 0.00 & 0.00 \\
PDEB (wt\%) & 0.00 & 1.14 & 99.92 & 99.92 & 99.92 & 0.15 & 0.15 & 0.00 & 0.13 & 0.12 & 2.23 \\
\hline & & & & & & & & & & &
\end{tabular}


S10. Case D results

Table S37 Optimization of the modified aromatics complex with crystallization at $244 \mathrm{~K}$ (D)

\begin{tabular}{lccccc} 
& Step 1 & Step 2 & Step 3 & Step 4 & Step 5 \\
\hline Profit $\left(\$ / t_{\text {Reformate }}\right)$ & 950.7 & 951.7 & 955.0 & 954.9 & 955.0 \\
$\Delta$ Revenue $\times 10^{-3}(\$ / \text { year })^{\mathrm{a}}$ & -1113 & -804 & 200 & 164 & 199 \\
Operation Cost $(\$ / \mathrm{h}):$ & & & & & \\
Aromatics Extraction & 353.6 & 353.6 & 353.6 & 353.6 & 353.6 \\
Benzene Column & 142.8 & 142.8 & 142.8 & 142.8 & 142.8 \\
Xylene Splitter & 294.7 & 295.3 & 295.9 & 295.8 & 295.9 \\
Extract Column & 202.8 & 183.2 & 121.2 & 122.8 & 121.2 \\
Raffinate Column & 490.2 & 480.9 & 436.9 & 438.8 & 437.0 \\
Isomerization & 362.7 & 358.3 & 354.2 & 354.6 & 354.1 \\
$o-X y l e n e ~ C o l u m n$ & 61.4 & 61.4 & 61.4 & 61.4 & 61.4 \\
Crystallization & 61.5 & 60.4 & 59.7 & 59.7 & 59.7 \\
SMB: & & & & & \\
Configuration & $5-9-7-3$ & $10-5-3-6$ & $10-5-3-6$ & $5-9-7-3$ & $10-4-4-6$ \\
Switching time $(\mathrm{s})$ & 66 & 66 & 180 & 180 & 180 \\
Productivity $\left(\mathrm{kg} / \mathrm{hm}{ }^{3}\right)$ & 47.99 & 47.55 & 47.22 & 47.26 & 47.23 \\
Desorbent consumption $(\mathrm{kg} / \mathrm{kg})$ & 5.35 & 4.75 & 1.74 & 1.82 & 1.74 \\
Recovery $(\%)$ & 98.25 & 99.13 & 99.99 & 99.89 & 99.99 \\
Total $\Delta \mathrm{P}(\mathrm{kPa})$ & 227 & 227 & 67 & 70 & 68 \\
Maximum $\Delta \mathrm{P} / \mathrm{bed}(\mathrm{kPa})$ & 10.4 & 10.4 & 3.7 & 3.7 & 3.7 \\
\hline
\end{tabular}

a Compared to the base case (A0) assuming 330 days of operation per year. 
Table S38 Mass balance for the modified aromatics complex with crystallization at $244 \mathrm{~K}$ after step 1 (D1)

\begin{tabular}{lccccccccccc}
\hline Stream & 1 & 2 & 3 & 4 & 5 & 6 & 7 & 8 & 9 & 10 & 11 \\
\hline Flow (t/h) & 38.10 & 20.60 & 17.50 & 63.93 & 57.96 & 43.74 & 71.36 & 7.26 & 4.89 & 15.71 & 10.30 \\
Ben (wt\%) & 12.86 & 23.78 & 0.00 & 0.00 & 0.00 & 0.00 & 0.00 & 0.00 & 99.88 & 0.08 & 0.00 \\
Tol (wt\%) & 41.21 & 76.22 & 0.00 & 0.00 & 0.00 & 0.00 & 0.00 & 0.00 & 0.12 & 99.92 & 0.00 \\
ECH (wt\%) & 0.00 & 0.00 & 0.00 & 18.18 & 20.67 & 0.83 & 16.29 & 0.00 & 0.00 & 0.00 & 0.00 \\
EB (wt\%) & 3.20 & 0.00 & 6.97 & 6.32 & 7.05 & 0.11 & 5.66 & 0.00 & 0.00 & 0.00 & 0.00 \\
PX (wt\%) & 6.56 & 0.00 & 14.28 & 16.40 & 18.75 & 24.41 & 0.27 & 0.00 & 0.00 & 0.00 & 100.00 \\
MX (wt\%) & 13.88 & 0.00 & 30.22 & 35.06 & 39.28 & 0.88 & 31.40 & 0.09 & 0.00 & 0.00 & 0.00 \\
OX (wt\%) & 6.29 & 0.00 & 13.69 & 14.41 & 14.22 & 0.29 & 11.40 & 15.15 & 0.00 & 0.00 & 0.00 \\
TMBen (wt\%) & 16.00 & 0.00 & 34.84 & 9.53 & 0.00 & 0.00 & 0.00 & 83.96 & 0.00 & 0.00 & 0.00 \\
PDEB (wt\%) & 0.00 & 0.00 & 0.00 & 0.09 & 0.03 & 73.48 & 34.99 & 0.80 & 0.00 & 0.00 & 0.00 \\
\hline Stream & 12 & 13 & 14 & 15 & 16 & 17 & 18 & 19 & 20 & 21 & 22 \\
\hline Flow (t/h) & 1.10 & 6.16 & 32.15 & 24.93 & 57.08 & 46.43 & 46.43 & 20.60 & 11.59 & 56.67 & 1.29 \\
Ben (wt\%) & 0.00 & 0.00 & 0.00 & 0.00 & 0.00 & 0.00 & 0.00 & 23.78 & 0.00 & 0.00 & 0.00 \\
Tol (wt\%) & 0.00 & 0.00 & 0.00 & 0.00 & 0.00 & 0.00 & 0.00 & 76.22 & 0.00 & 0.00 & 0.00 \\
ECH (wt\%) & 0.00 & 0.00 & 0.00 & 0.00 & 0.00 & 25.03 & 25.03 & 0.00 & 3.12 & 20.51 & 27.98 \\
EB (wt\%) & 0.00 & 0.00 & 0.00 & 0.00 & 0.00 & 8.70 & 6.07 & 0.00 & 0.42 & 7.13 & 3.74 \\
PX (wt\%) & 0.00 & 0.00 & 0.00 & 0.00 & 0.00 & 0.41 & 17.20 & 0.00 & 92.16 & 18.50 & 29.62 \\
MX (wt\%) & 0.59 & 0.00 & 0.08 & 0.00 & 0.04 & 48.26 & 36.89 & 0.00 & 3.09 & 39.54 & 27.71 \\
OX (wt\%) & 99.29 & 0.09 & 0.00 & 0.08 & 0.03 & 17.48 & 14.68 & 0.00 & 1.09 & 14.32 & 9.82 \\
TMBen (wt\%) & 0.11 & 98.97 & 0.00 & 0.00 & 0.00 & 0.00 & 0.00 & 0.00 & 0.00 & 0.00 & 0.00 \\
PDEB (wt\%) & 0.00 & 0.94 & 99.92 & 99.92 & 99.92 & 0.12 & 0.12 & 0.00 & 0.13 & 0.00 & 1.13 \\
\hline & & & & & & & & & & &
\end{tabular}


Table S39 Mass balance for the modified aromatics complex with crystallization at $244 \mathrm{~K}$ after step 2 (D2)

\begin{tabular}{lccccccccccc}
\hline Stream & 1 & 2 & 3 & 4 & 5 & 6 & 7 & 8 & 9 & 10 & 11 \\
\hline Flow (t/h) & 38.10 & 20.60 & 17.50 & 63.36 & 57.08 & 37.96 & 69.40 & 7.26 & 4.89 & 15.71 & 10.30 \\
Ben (wt\%) & 12.86 & 23.78 & 0.00 & 0.00 & 0.00 & 0.00 & 0.00 & 0.00 & 99.88 & 0.08 & 0.00 \\
Tol (wt\%) & 41.21 & 76.22 & 0.00 & 0.00 & 0.00 & 0.00 & 0.00 & 0.00 & 0.12 & 99.92 & 0.00 \\
ECH (wt\%) & 0.00 & 0.00 & 0.00 & 18.12 & 20.71 & 0.90 & 16.54 & 0.00 & 0.00 & 0.00 & 0.00 \\
EB (wt\%) & 3.20 & 0.00 & 6.97 & 6.32 & 7.08 & 0.10 & 5.77 & 0.00 & 0.00 & 0.00 & 0.00 \\
PX (wt\%) & 6.56 & 0.00 & 14.28 & 16.40 & 18.70 & 27.87 & 0.13 & 0.00 & 0.00 & 0.00 & 100.00 \\
MX (wt\%) & 13.88 & 0.00 & 30.22 & 35.05 & 39.28 & 0.63 & 31.99 & 0.09 & 0.00 & 0.00 & 0.00 \\
OX (wt\%) & 6.29 & 0.00 & 13.69 & 14.41 & 14.21 & 0.21 & 11.60 & 15.15 & 0.00 & 0.00 & 0.00 \\
TMBen (wt\%) & 16.00 & 0.00 & 34.84 & 9.62 & 0.00 & 0.00 & 0.00 & 83.97 & 0.00 & 0.00 & 0.00 \\
PDEB (wt\%) & 0.00 & 0.00 & 0.00 & 0.09 & 0.02 & 70.29 & 33.97 & 0.79 & 0.00 & 0.00 & 0.00 \\
\hline Stream & 12 & 13 & 14 & 15 & 16 & 17 & 18 & 19 & 20 & 21 & 22 \\
\hline Flow (t/h) & 1.10 & 6.16 & 26.69 & 23.53 & 50.22 & 45.86 & 45.86 & 20.60 & 11.27 & 56.10 & 0.98 \\
Ben (wt\%) & 0.00 & 0.00 & 0.00 & 0.00 & 0.00 & 0.00 & 0.00 & 23.78 & 0.00 & 0.00 & 0.00 \\
Tol (wt\%) & 0.00 & 0.00 & 0.00 & 0.00 & 0.00 & 0.00 & 0.00 & 76.22 & 0.00 & 0.00 & 0.00 \\
ECH (wt\%) & 0.00 & 0.00 & 0.00 & 0.00 & 0.00 & 25.03 & 25.03 & 0.00 & 3.03 & 20.46 & 34.97 \\
EB (wt\%) & 0.00 & 0.00 & 0.00 & 0.00 & 0.00 & 8.73 & 6.07 & 0.00 & 0.32 & 7.14 & 3.72 \\
PX (wt\%) & 0.00 & 0.00 & 0.00 & 0.00 & 0.00 & 0.20 & 17.20 & 0.00 & 93.87 & 18.52 & 29.19 \\
MX (wt\%) & 0.59 & 0.00 & 0.08 & 0.00 & 0.04 & 48.40 & 36.89 & 0.00 & 1.95 & 39.57 & 22.48 \\
OX (wt\%) & 99.29 & 0.09 & 0.00 & 0.08 & 0.04 & 17.51 & 14.68 & 0.00 & 0.71 & 14.32 & 8.19 \\
TMBen (wt\%) & 0.11 & 98.98 & 0.00 & 0.00 & 0.00 & 0.00 & 0.00 & 0.00 & 0.00 & 0.00 & 0.00 \\
PDEB (wt\%) & 0.00 & 0.93 & 99.92 & 99.92 & 99.92 & 0.12 & 0.12 & 0.00 & 0.13 & 0.00 & 1.46 \\
\hline & & & & & & & & & & &
\end{tabular}


Table S40 Mass balance for the modified aromatics complex with crystallization at $244 \mathrm{~K}$ after step 3 (D3)

\begin{tabular}{lccccccccccc}
\hline Stream & 1 & 2 & 3 & 4 & 5 & 6 & 7 & 8 & 9 & 10 & 11 \\
\hline Flow (t/h) & 38.10 & 20.60 & 17.50 & 62.83 & 56.33 & 18.15 & 56.51 & 7.26 & 4.89 & 15.71 & 10.30 \\
Ben (wt\%) & 12.86 & 23.78 & 0.00 & 0.00 & 0.00 & 0.00 & 0.00 & 0.00 & 99.88 & 0.08 & 0.00 \\
Tol (wt\%) & 41.21 & 76.22 & 0.00 & 0.00 & 0.00 & 0.00 & 0.00 & 0.00 & 0.12 & 99.92 & 0.00 \\
ECH (wt\%) & 0.00 & 0.00 & 0.00 & 18.06 & 20.85 & 2.21 & 20.08 & 0.00 & 0.00 & 0.00 & 0.00 \\
EB (wt\%) & 3.20 & 0.00 & 6.97 & 6.32 & 7.05 & 0.01 & 7.03 & 0.00 & 0.00 & 0.00 & 0.00 \\
PX (wt\%) & 6.56 & 0.00 & 14.28 & 16.39 & 18.65 & 57.90 & 0.00 & 0.00 & 0.00 & 0.00 & 100.00 \\
MX (wt\%) & 13.88 & 0.00 & 30.22 & 35.03 & 39.25 & 0.65 & 38.94 & 0.09 & 0.00 & 0.00 & 0.00 \\
OX (wt\%) & 6.29 & 0.00 & 13.69 & 14.41 & 14.16 & 0.14 & 14.09 & 15.15 & 0.00 & 0.00 & 0.00 \\
TMBen (wt\%) & 16.00 & 0.00 & 34.84 & 9.70 & 0.00 & 0.00 & 0.00 & 83.98 & 0.00 & 0.00 & 0.00 \\
PDEB (wt\%) & 0.00 & 0.00 & 0.00 & 0.09 & 0.02 & 39.10 & 19.87 & 0.78 & 0.00 & 0.00 & 0.00 \\
\hline Stream & 12 & 13 & 14 & 15 & 16 & 17 & 18 & 19 & 20 & 21 & 22 \\
\hline Flow (t/h) & 1.10 & 6.16 & 7.09 & 11.18 & 18.27 & 45.33 & 45.33 & 20.60 & 11.06 & 55.57 & 0.77 \\
Ben (wt\%) & 0.00 & 0.00 & 0.00 & 0.00 & 0.00 & 0.00 & 0.00 & 23.78 & 0.00 & 0.00 & 0.00 \\
Tol (wt\%) & 0.00 & 0.00 & 0.00 & 0.00 & 0.00 & 0.00 & 0.00 & 76.22 & 0.00 & 0.00 & 0.00 \\
ECH (wt\%) & 0.00 & 0.00 & 0.00 & 0.00 & 0.00 & 25.03 & 25.03 & 0.00 & 3.62 & 20.42 & 52.35 \\
EB (wt\%) & 0.00 & 0.00 & 0.00 & 0.00 & 0.00 & 8.76 & 6.07 & 0.00 & 0.02 & 7.15 & 0.32 \\
PX (wt\%) & 0.00 & 0.00 & 0.00 & 0.00 & 0.00 & 0.00 & 17.20 & 0.00 & 95.00 & 18.53 & 27.69 \\
MX (wt\%) & 0.59 & 0.00 & 0.08 & 0.00 & 0.03 & 48.54 & 36.89 & 0.00 & 1.01 & 39.59 & 14.62 \\
OX (wt\%) & 99.29 & 0.09 & 0.00 & 0.08 & 0.05 & 17.54 & 14.68 & 0.00 & 0.22 & 14.31 & 3.20 \\
TMBen (wt\%) & 0.11 & 98.99 & 0.00 & 0.00 & 0.00 & 0.00 & 0.00 & 0.00 & 0.00 & 0.00 & 0.00 \\
PDEB (wt\%) & 0.00 & 0.92 & 99.92 & 99.92 & 99.92 & 0.12 & 0.12 & 0.00 & 0.13 & 0.00 & 1.82 \\
\hline & & & & & & & & & & &
\end{tabular}


Table S41 Mass balance for the modified aromatics complex with crystallization at $244 \mathrm{~K}$ after step 4 (D4)

\begin{tabular}{lccccccccccc}
\hline Stream & 1 & 2 & 3 & 4 & 5 & 6 & 7 & 8 & 9 & 10 & 11 \\
\hline Flow (t/h) & 38.10 & 20.60 & 17.50 & 62.88 & 56.40 & 18.62 & 56.99 & 7.26 & 4.89 & 15.71 & 10.30 \\
Ben (wt\%) & 12.86 & 23.78 & 0.00 & 0.00 & 0.00 & 0.00 & 0.00 & 0.00 & 99.88 & 0.08 & 0.00 \\
Tol (wt\%) & 41.21 & 76.22 & 0.00 & 0.00 & 0.00 & 0.00 & 0.00 & 0.00 & 0.12 & 99.92 & 0.00 \\
ECH (wt\%) & 0.00 & 0.00 & 0.00 & 18.07 & 20.74 & 1.83 & 19.93 & 0.00 & 0.00 & 0.00 & 0.00 \\
EB (wt\%) & 3.20 & 0.00 & 6.97 & 6.32 & 7.06 & 0.03 & 6.97 & 0.00 & 0.00 & 0.00 & 0.00 \\
PX (wt\%) & 6.56 & 0.00 & 14.28 & 16.39 & 18.67 & 56.49 & 0.02 & 0.00 & 0.00 & 0.00 & 100.00 \\
MX (wt\%) & 13.88 & 0.00 & 30.22 & 35.03 & 39.32 & 0.85 & 38.64 & 0.09 & 0.00 & 0.00 & 0.00 \\
OX (wt\%) & 6.29 & 0.00 & 13.69 & 14.41 & 14.19 & 0.23 & 13.99 & 15.15 & 0.00 & 0.00 & 0.00 \\
TMBen (wt\%) & 16.00 & 0.00 & 34.84 & 9.69 & 0.00 & 0.00 & 0.00 & 83.98 & 0.00 & 0.00 & 0.00 \\
PDEB (wt\%) & 0.00 & 0.00 & 0.00 & 0.09 & 0.02 & 40.58 & 20.44 & 0.78 & 0.00 & 0.00 & 0.00 \\
\hline Stream & 12 & 13 & 14 & 15 & 16 & 17 & 18 & 19 & 20 & 21 & 22 \\
\hline Flow (t/h) & 1.10 & 6.16 & 7.55 & 11.60 & 19.15 & 45.39 & 45.39 & 20.60 & 11.07 & 55.63 & 0.77 \\
Ben (wt\%) & 0.00 & 0.00 & 0.00 & 0.00 & 0.00 & 0.00 & 0.00 & 23.78 & 0.00 & 0.00 & 0.00 \\
Tol (wt\%) & 0.00 & 0.00 & 0.00 & 0.00 & 0.00 & 0.00 & 0.00 & 76.22 & 0.00 & 0.00 & 0.00 \\
ECH (wt\%) & 0.00 & 0.00 & 0.00 & 0.00 & 0.00 & 25.03 & 25.03 & 0.00 & 3.07 & 20.42 & 43.88 \\
EB (wt\%) & 0.00 & 0.00 & 0.00 & 0.00 & 0.00 & 8.76 & 6.07 & 0.00 & 0.05 & 7.14 & 0.66 \\
PX (wt\%) & 0.00 & 0.00 & 0.00 & 0.00 & 0.00 & 0.02 & 17.20 & 0.00 & 95.00 & 18.53 & 28.51 \\
MX (wt\%) & 0.59 & 0.00 & 0.08 & 0.00 & 0.03 & 48.52 & 36.89 & 0.00 & 1.38 & 39.59 & 19.73 \\
OX (wt\%) & 99.29 & 0.09 & 0.00 & 0.08 & 0.05 & 17.54 & 14.68 & 0.00 & 0.38 & 14.31 & 5.41 \\
TMBen (wt\%) & 0.11 & 98.99 & 0.00 & 0.00 & 0.00 & 0.00 & 0.00 & 0.00 & 0.00 & 0.00 & 0.00 \\
PDEB (wt\%) & 0.00 & 0.92 & 99.92 & 99.92 & 99.92 & 0.12 & 0.12 & 0.00 & 0.13 & 0.00 & 1.80 \\
\hline & & & & & & & & & & &
\end{tabular}


Table S42 Mass balance for the modified aromatics complex with crystallization at $244 \mathrm{~K}$ after step 5 (D5)

\begin{tabular}{lccccccccccc}
\hline Stream & 1 & 2 & 3 & 4 & 5 & 6 & 7 & 8 & 9 & 10 & 11 \\
\hline Flow (t/h) & 38.10 & 20.60 & 17.50 & 62.82 & 56.34 & 18.13 & 56.53 & 7.26 & 4.89 & 15.71 & 10.30 \\
Ben (wt\%) & 12.86 & 23.78 & 0.00 & 0.00 & 0.00 & 0.00 & 0.00 & 0.00 & 99.88 & 0.08 & 0.00 \\
Tol (wt\%) & 41.21 & 76.22 & 0.00 & 0.00 & 0.00 & 0.00 & 0.00 & 0.00 & 0.12 & 99.92 & 0.00 \\
ECH (wt\%) & 0.00 & 0.00 & 0.00 & 18.06 & 20.85 & 2.21 & 20.07 & 0.00 & 0.00 & 0.00 & 0.00 \\
EB (wt\%) & 3.20 & 0.00 & 6.97 & 6.32 & 7.06 & 0.03 & 7.02 & 0.00 & 0.00 & 0.00 & 0.00 \\
PX (wt\%) & 6.56 & 0.00 & 14.28 & 16.39 & 18.66 & 57.95 & 0.00 & 0.00 & 0.00 & 0.00 & 100.00 \\
MX (wt\%) & 13.88 & 0.00 & 30.22 & 35.03 & 39.25 & 0.65 & 38.92 & 0.09 & 0.00 & 0.00 & 0.00 \\
OX (wt\%) & 6.29 & 0.00 & 13.69 & 14.41 & 14.16 & 0.14 & 14.08 & 15.15 & 0.00 & 0.00 & 0.00 \\
TMBen (wt\%) & 16.00 & 0.00 & 34.84 & 9.70 & 0.00 & 0.00 & 0.00 & 83.98 & 0.00 & 0.00 & 0.00 \\
PDEB (wt\%) & 0.00 & 0.00 & 0.00 & 0.09 & 0.02 & 39.02 & 19.90 & 0.78 & 0.00 & 0.00 & 0.00 \\
\hline Stream & 12 & 13 & 14 & 15 & 16 & 17 & 18 & 19 & 20 & 21 & 22 \\
\hline Flow (t/h) & 1.10 & 6.16 & 7.07 & 11.20 & 18.27 & 45.33 & 45.33 & 20.60 & 11.07 & 55.56 & 0.77 \\
Ben (wt\%) & 0.00 & 0.00 & 0.00 & 0.00 & 0.00 & 0.00 & 0.00 & 23.78 & 0.00 & 0.00 & 0.00 \\
Tol (wt\%) & 0.00 & 0.00 & 0.00 & 0.00 & 0.00 & 0.00 & 0.00 & 76.22 & 0.00 & 0.00 & 0.00 \\
ECH (wt\%) & 0.00 & 0.00 & 0.00 & 0.00 & 0.00 & 25.03 & 25.03 & 0.00 & 3.62 & 20.42 & 51.90 \\
EB (wt\%) & 0.00 & 0.00 & 0.00 & 0.00 & 0.00 & 8.76 & 6.07 & 0.00 & 0.05 & 7.15 & 0.74 \\
PX (wt\%) & 0.00 & 0.00 & 0.00 & 0.00 & 0.00 & 0.00 & 17.20 & 0.00 & 94.97 & 18.53 & 27.74 \\
MX (wt\%) & 0.59 & 0.00 & 0.08 & 0.00 & 0.03 & 48.54 & 36.89 & 0.00 & 1.01 & 39.59 & 14.57 \\
OX (wt\%) & 99.29 & 0.09 & 0.00 & 0.08 & 0.05 & 17.54 & 14.68 & 0.00 & 0.23 & 14.31 & 3.24 \\
TMBen (wt\%) & 0.11 & 98.99 & 0.00 & 0.00 & 0.00 & 0.00 & 0.00 & 0.00 & 0.00 & 0.00 & 0.00 \\
PDEB (wt\%) & 0.00 & 0.92 & 99.92 & 99.92 & 99.92 & 0.12 & 0.12 & 0.00 & 0.13 & 0.00 & 1.81 \\
\hline & & & & & & & & & & &
\end{tabular}




\section{S11. Case E results}

Table S43 Optimization of the modified aromatics complex with toluene methylation and crystallization at $218 \mathrm{~K}(\mathrm{E})$

\begin{tabular}{|c|c|c|c|c|c|}
\hline & Step 1 & Step 2 & Step 3 & Step 4 & Step 5 \\
\hline Profit $\left(\$ / t_{\text {Reformate }}\right)$ & 1037.5 & 1040.1 & 1043.9 & 1043.7 & 1043.9 \\
\hline$\Delta$ Revenue $\times 10^{-3}(\$ / \text { year })^{\mathrm{a}}$ & 25085 & 25858 & 27001 & 26955 & 27006 \\
\hline \multicolumn{6}{|l|}{ Operation Cost $(\$ / \mathrm{h})$ : } \\
\hline Aromatics Extraction & 353.6 & 353.6 & 353.6 & 353.6 & 353.6 \\
\hline Benzene Column & 142.8 & 142.8 & 142.8 & 142.8 & 142.8 \\
\hline Xylene Splitter & 322.8 & 319.9 & 317.4 & 317.7 & 317.4 \\
\hline Extract Column & 208.4 & 196.4 & 123.8 & 125.9 & 123.8 \\
\hline Raffinate Column & 539.5 & 524.4 & 486.3 & 487.8 & 486.4 \\
\hline Isomerization & 411.6 & 406.3 & 401.7 & 402.3 & 401.7 \\
\hline$o$-Xylene Column & 63.1 & 62.9 & 62.8 & 62.8 & 62.8 \\
\hline Crystallization & 268.8 & 268.7 & 266.0 & 266.2 & 265.9 \\
\hline Toluene Methylation & 1127.6 & 1074.2 & 1076.0 & 1076.0 & 1075.6 \\
\hline Toluene Column & 869.1 & 861.1 & 863.1 & 863.1 & 863.1 \\
\hline \multicolumn{6}{|l|}{ SMB: } \\
\hline Configuration & $5-9-7-3$ & $9-5-4-6$ & $9-5-4-6$ & $5-9-7-3$ & $8-4-4-8$ \\
\hline Switching time (s) & 66 & 66 & 180 & 180 & 180 \\
\hline Productivity $\left(\mathrm{kg} / \mathrm{hm}^{3}\right)$ & 51.22 & 51.22 & 50.93 & 50.96 & 50.93 \\
\hline Desorbent consumption $(\mathrm{kg} / \mathrm{kg})$ & 5.36 & 4.76 & 1.78 & 1.86 & 1.78 \\
\hline Recovery (\%) & 98.46 & 99.50 & 99.96 & 99.89 & 99.95 \\
\hline Total $\Delta \mathrm{P}(\mathrm{kPa})$ & 228 & 228 & 68 & 70 & 68 \\
\hline Maximum $\Delta \mathrm{P} /$ bed $(\mathrm{kPa})$ & 10.6 & 10.6 & 3.8 & 3.8 & 3.8 \\
\hline \multicolumn{6}{|l|}{ Toluene Methylation: } \\
\hline Toluene conversion $(\%)$ & 20.42 & 20.59 & 20.55 & 20.55 & 20.55 \\
\hline$p$-Xylene selectivity (\%) & 94.45 & 94.45 & 94.44 & 94.44 & 94.44 \\
\hline Toluene/MeOH (mol/mol) & 1.67 & 1.69 & 1.70 & 1.70 & 1.70 \\
\hline Water/Hydrocarbons (mol/mol) & 0.33 & 0.29 & 0.29 & 0.29 & 0.29 \\
\hline Space time $\left(\mathrm{g}_{\mathrm{cat}} \mathrm{h} / \mathrm{mol}_{\mathrm{Tol}}\right)$ & 3.62 & 3.49 & 3.49 & 3.49 & 3.49 \\
\hline $\mathrm{MeOH}$ loss $(\%)$ & 36.58 & 37.03 & 36.84 & 36.84 & 36.84 \\
\hline
\end{tabular}

a Compared to the base case (A0) assuming 330 days of operation per year. 
Table S44 Mass balance for the modified aromatics complex with toluene methylation and crystallization at 218 K after step 1 (E1)

\begin{tabular}{|c|c|c|c|c|c|c|c|c|c|c|c|c|c|c|}
\hline Stream & 1 & 2 & 3 & 4 & 5 & 6 & 7 & 8 & 9 & 10 & 11 & 12 & 13 & 14 \\
\hline Flow $(\mathrm{t} / \mathrm{h})$ & 38.10 & 20.60 & 17.50 & 70.14 & 62.75 & 44.76 & 79.08 & 7.39 & 4.89 & 60.84 & 27.32 & 1.22 & 6.18 & 32.80 \\
\hline $\operatorname{Ben}(w t \%)$ & 12.86 & 23.78 & 0.00 & 0.00 & 0.00 & 0.00 & 0.00 & 0.00 & 99.88 & 0.11 & 0.00 & 0.00 & 0.00 & 0.00 \\
\hline Tol (wt\%) & 41.21 & 76.22 & 0.00 & 0.02 & 0.02 & 0.00 & 0.00 & 0.00 & 0.12 & 99.78 & 0.00 & 0.00 & 0.00 & 0.00 \\
\hline $\mathrm{ECH}(\mathrm{wt} \%)$ & 0.00 & 0.00 & 0.00 & 18.79 & 21.00 & 0.52 & 16.37 & 0.00 & 0.00 & 0.00 & 0.00 & 0.00 & 0.00 & 0.00 \\
\hline EB (wt\%) & 3.20 & 0.00 & 6.97 & 6.29 & 7.03 & 0.06 & 5.55 & 0.00 & 0.00 & 0.00 & 0.00 & 0.00 & 0.00 & 0.00 \\
\hline $\mathrm{PX}(\mathrm{wt} \%)$ & 6.56 & 0.00 & 14.28 & 16.47 & 18.41 & 25.46 & 0.23 & 0.00 & 0.00 & 0.12 & 100.00 & 0.00 & 0.00 & 0.08 \\
\hline $\mathrm{MX}(w \mathrm{t} \%)$ & 13.88 & 0.00 & 30.22 & 35.20 & 39.34 & 0.50 & 30.94 & 0.09 & 0.00 & 0.00 & 0.00 & 0.55 & 0.00 & 0.00 \\
\hline OX (wt\%) & 6.29 & 0.00 & 13.69 & 14.43 & 14.19 & 0.19 & 11.19 & 16.42 & 0.00 & 0.00 & 0.00 & 99.34 & 0.09 & 0.00 \\
\hline TMBen (wt\%) & 16.00 & 0.00 & 34.84 & 8.69 & 0.00 & 0.00 & 0.00 & 82.42 & 0.00 & 0.00 & 0.00 & 0.11 & 98.64 & 0.00 \\
\hline PDEB (wt\%) & 0.00 & 0.00 & 0.00 & 0.11 & 0.00 & 73.27 & 35.73 & 1.06 & 0.00 & 0.00 & 0.00 & 0.00 & 1.27 & 99.92 \\
\hline Stream & 15 & 16 & 17 & 18 & 19 & 20 & 21 & 22 & 23 & 24 & 25 & 26 & 27 & \\
\hline Flow $(\mathrm{t} / \mathrm{h})$ & 28.21 & 61.02 & 52.65 & 52.65 & 20.60 & 29.09 & 50.87 & 1.78 & 76.55 & 77.99 & 15.71 & 11.95 & 17.14 & \\
\hline Ben (wt $\%)$ & 0.00 & 0.00 & 0.00 & 0.00 & 23.78 & 0.00 & 0.00 & 0.00 & 0.10 & 0.08 & 0.08 & 0.00 & 0.00 & \\
\hline Tol (wt\%) & 0.00 & 0.00 & 0.03 & 0.03 & 76.22 & 0.05 & 0.00 & 0.84 & 99.81 & 77.87 & 99.92 & 0.00 & 0.09 & \\
\hline $\mathrm{ECH}(\mathrm{wt} \%)$ & 0.00 & 0.00 & 25.03 & 25.03 & 0.00 & 0.80 & 25.45 & 13.07 & 0.00 & 0.00 & 0.00 & 1.94 & 0.00 & \\
\hline EB (wt\%) & 0.00 & 0.00 & 8.38 & 6.07 & 0.00 & 0.10 & 8.62 & 1.60 & 0.00 & 0.00 & 0.00 & 0.24 & 0.00 & \\
\hline $\mathrm{PX}(\mathrm{wt} \%)$ & 0.00 & 0.04 & 0.77 & 17.19 & 0.00 & 94.67 & 0.35 & 12.77 & 0.09 & 20.83 & 0.00 & 95.13 & 94.35 & \\
\hline MX (wt\%) & 0.00 & 0.00 & 47.81 & 36.86 & 0.00 & 2.41 & 48.10 & 39.37 & 0.00 & 0.61 & 0.00 & 1.86 & 2.78 & \\
\hline OX $(w t \%)$ & 0.08 & 0.04 & 17.83 & 14.67 & 0.00 & 1.93 & 17.35 & 31.51 & 0.00 & 0.61 & 0.00 & 0.70 & 2.78 & \\
\hline TMBen (wt\%) & 0.00 & 0.00 & 0.00 & 0.00 & 0.00 & 0.00 & 0.00 & 0.00 & 0.00 & 0.00 & 0.00 & 0.00 & 0.00 & \\
\hline PDEB (wt\%) & 99.92 & 99.92 & 0.15 & 0.15 & 0.00 & 0.05 & 0.12 & 0.85 & 0.00 & 0.00 & 0.00 & 0.13 & 0.00 & \\
\hline
\end{tabular}


Table S45 Mass balance for the modified aromatics complex with toluene methylation and crystallization at $218 \mathrm{~K}$ after step 2 (E2)

\begin{tabular}{|c|c|c|c|c|c|c|c|c|c|c|c|c|c|c|}
\hline Stream & 1 & 2 & 3 & 4 & 5 & 6 & 7 & 8 & 9 & 10 & 11 & 12 & 13 & 14 \\
\hline Flow $(\mathrm{t} / \mathrm{h})$ & 38.10 & 20.60 & 17.50 & 69.46 & 62.08 & 40.99 & 75.36 & 7.38 & 4.89 & 60.20 & 27.34 & 1.21 & 6.18 & 29.09 \\
\hline $\operatorname{Ben}(w t \%)$ & 12.86 & 23.78 & 0.00 & 0.00 & 0.00 & 0.00 & 0.00 & 0.00 & 99.88 & 0.11 & 0.00 & 0.00 & 0.00 & 0.00 \\
\hline Tol (wt\%) & 41.21 & 76.22 & 0.00 & 0.02 & 0.02 & 0.00 & 0.00 & 0.00 & 0.12 & 99.78 & 0.00 & 0.00 & 0.00 & 0.00 \\
\hline $\mathrm{ECH}(\mathrm{wt} \%)$ & 0.00 & 0.00 & 0.00 & 18.72 & 20.95 & 0.60 & 16.94 & 0.00 & 0.00 & 0.00 & 0.00 & 0.00 & 0.00 & 0.00 \\
\hline EB (wt\%) & 3.20 & 0.00 & 6.97 & 6.29 & 7.04 & 0.10 & 5.75 & 0.00 & 0.00 & 0.00 & 0.00 & 0.00 & 0.00 & 0.00 \\
\hline $\mathrm{PX}(\mathrm{wt} \%)$ & 6.56 & 0.00 & 14.28 & 16.46 & 18.41 & 27.81 & 0.08 & 0.00 & 0.00 & 0.12 & 100.00 & 0.00 & 0.00 & 0.08 \\
\hline $\mathrm{MX}(w \mathrm{t} \%)$ & 13.88 & 0.00 & 30.22 & 35.19 & 39.36 & 0.40 & 32.22 & 0.09 & 0.00 & 0.00 & 0.00 & 0.55 & 0.00 & 0.00 \\
\hline OX $(w t \%)$ & 6.29 & 0.00 & 13.69 & 14.43 & 14.21 & 0.16 & 11.64 & 16.29 & 0.00 & 0.00 & 0.00 & 99.33 & 0.09 & 0.00 \\
\hline TMBen (wt\%) & 16.00 & 0.00 & 34.84 & 8.78 & 0.00 & 0.00 & 0.00 & 82.57 & 0.00 & 0.00 & 0.00 & 0.11 & 98.65 & 0.00 \\
\hline PDEB (wt\%) & 0.00 & 0.00 & 0.00 & 0.11 & 0.00 & 70.94 & 33.37 & 1.05 & 0.00 & 0.00 & 0.00 & 0.00 & 1.26 & 99.92 \\
\hline Stream & 15 & 16 & 17 & 18 & 19 & 20 & 21 & 22 & 23 & 24 & 25 & 26 & 27 & \\
\hline Flow $(\mathrm{t} / \mathrm{h})$ & 25.11 & 54.19 & 51.96 & 51.96 & 20.60 & 29.05 & 50.25 & 1.71 & 75.90 & 77.35 & 15.71 & 11.90 & 17.15 & \\
\hline Ben (wt $\%)$ & 0.00 & 0.00 & 0.00 & 0.00 & 23.78 & 0.00 & 0.00 & 0.00 & 0.10 & 0.08 & 0.08 & 0.00 & 0.00 & \\
\hline Tol (wt\%) & 0.00 & 0.00 & 0.03 & 0.03 & 76.22 & 0.05 & 0.00 & 0.87 & 99.81 & 77.67 & 99.92 & 0.00 & 0.09 & \\
\hline $\mathrm{ECH}(\mathrm{wt} \%)$ & 0.00 & 0.00 & 25.03 & 25.03 & 0.00 & 0.84 & 25.40 & 14.24 & 0.00 & 0.00 & 0.00 & 2.05 & 0.00 & \\
\hline EB (wt\%) & 0.00 & 0.00 & 8.41 & 6.07 & 0.00 & 0.13 & 8.62 & 2.27 & 0.00 & 0.00 & 0.00 & 0.33 & 0.00 & \\
\hline $\mathrm{PX}(\mathrm{wt} \%)$ & 0.00 & 0.04 & 0.53 & 17.19 & 0.00 & 94.85 & 0.11 & 12.74 & 0.09 & 21.01 & 0.00 & 95.57 & 94.35 & \\
\hline MX (wt\%) & 0.00 & 0.00 & 47.95 & 36.86 & 0.00 & 2.20 & 48.31 & 37.31 & 0.00 & 0.62 & 0.00 & 1.37 & 2.78 & \\
\hline OX $(w t \%)$ & 0.08 & 0.04 & 17.89 & 14.67 & 0.00 & 1.87 & 17.42 & 31.69 & 0.00 & 0.62 & 0.00 & 0.56 & 2.78 & \\
\hline TMBen (wt\%) & 0.00 & 0.00 & 0.00 & 0.00 & 0.00 & 0.00 & 0.00 & 0.00 & 0.00 & 0.00 & 0.00 & 0.00 & 0.00 & \\
\hline PDEB (wt\%) & 99.92 & 99.92 & 0.15 & 0.15 & 0.00 & 0.05 & 0.12 & 0.88 & 0.00 & 0.00 & 0.00 & 0.13 & 0.00 & \\
\hline
\end{tabular}


Table S46 Mass balance for the modified aromatics complex with toluene methylation and crystallization at $218 \mathrm{~K}$ after step 3 (E3)

\begin{tabular}{|c|c|c|c|c|c|c|c|c|c|c|c|c|c|c|}
\hline Stream & 1 & 2 & 3 & 4 & 5 & 6 & 7 & 8 & 9 & 10 & 11 & 12 & 13 & 14 \\
\hline Flow $(\mathrm{t} / \mathrm{h})$ & 38.10 & 20.60 & 17.50 & 68.88 & 61.51 & 18.12 & 63.52 & 7.37 & 4.89 & 60.36 & 27.35 & 1.19 & 6.18 & 6.70 \\
\hline Ben (wt\%) & 12.86 & 23.78 & 0.00 & 0.00 & 0.00 & 0.00 & 0.00 & 0.00 & 99.88 & 0.11 & 0.00 & 0.00 & 0.00 & 0.00 \\
\hline Tol (wt\%) & 41.21 & 76.22 & 0.00 & 0.02 & 0.02 & 0.00 & 0.00 & 0.00 & 0.12 & 99.78 & 0.00 & 0.00 & 0.00 & 0.00 \\
\hline $\mathrm{ECH}(\mathrm{wt} \%)$ & 0.00 & 0.00 & 0.00 & 18.67 & 20.91 & 0.37 & 20.15 & 0.00 & 0.00 & 0.00 & 0.00 & 0.00 & 0.00 & 0.00 \\
\hline $\mathrm{EB}(\mathrm{wt} \%)$ & 3.20 & 0.00 & 6.97 & 6.30 & 7.05 & 0.01 & 6.83 & 0.00 & 0.00 & 0.00 & 0.00 & 0.00 & 0.00 & 0.00 \\
\hline $\mathrm{PX}(\mathrm{wt} \%)$ & 6.56 & 0.00 & 14.28 & 16.45 & 18.42 & 62.53 & 0.01 & 0.00 & 0.00 & 0.12 & 100.00 & 0.00 & 0.00 & 0.08 \\
\hline MX (wt\%) & 13.88 & 0.00 & 30.22 & 35.17 & 39.38 & 0.02 & 38.14 & 0.09 & 0.00 & 0.00 & 0.00 & 0.56 & 0.00 & 0.00 \\
\hline $\mathrm{OX}(\mathrm{wt} \%)$ & 6.29 & 0.00 & 13.69 & 14.43 & 14.22 & 0.05 & 13.77 & 16.18 & 0.00 & 0.00 & 0.00 & 99.33 & 0.09 & 0.00 \\
\hline TMBen (wt\%) & 16.00 & 0.00 & 34.84 & 8.85 & 0.00 & 0.00 & 0.00 & 82.69 & 0.00 & 0.00 & 0.00 & 0.11 & 98.67 & 0.00 \\
\hline PDEB (wt\%) & 0.00 & 0.00 & 0.00 & 0.11 & 0.00 & 37.03 & 21.11 & 1.04 & 0.00 & 0.00 & 0.00 & 0.00 & 1.25 & 99.92 \\
\hline Stream & 15 & 16 & 17 & 18 & 19 & 20 & 21 & 22 & 23 & 24 & 25 & 26 & 27 & \\
\hline Flow $(\mathrm{t} / \mathrm{h})$ & 13.36 & 20.06 & 51.38 & 51.38 & 20.60 & 28.57 & 50.16 & 1.22 & 76.07 & 77.51 & 15.71 & 11.42 & 17.15 & \\
\hline Ben $(w t \%)$ & 0.00 & 0.00 & 0.00 & 0.00 & 23.78 & 0.00 & 0.00 & 0.00 & 0.10 & 0.08 & 0.08 & 0.00 & 0.00 & \\
\hline Tol (wt\%) & 0.00 & 0.00 & 0.03 & 0.03 & 76.22 & 0.05 & 0.00 & 1.22 & 99.81 & 77.73 & 99.92 & 0.00 & 0.09 & \\
\hline $\mathrm{ECH}(\mathrm{wt} \%)$ & 0.00 & 0.00 & 25.04 & 25.03 & 0.00 & 0.23 & 25.51 & 5.47 & 0.00 & 0.00 & 0.00 & 0.58 & 0.00 & \\
\hline $\mathrm{EB}(\mathrm{wt} \%)$ & 0.00 & 0.00 & 8.44 & 6.07 & 0.00 & 0.00 & 8.64 & 0.09 & 0.00 & 0.00 & 0.00 & 0.01 & 0.00 & \\
\hline $\mathrm{PX}(\mathrm{wt} \%)$ & 0.00 & 0.03 & 0.31 & 17.19 & 0.00 & 96.27 & 0.01 & 12.87 & 0.09 & 20.96 & 0.00 & 99.18 & 94.34 & \\
\hline MX (wt\%) & 0.00 & 0.00 & 48.08 & 36.86 & 0.00 & 1.68 & 48.29 & 39.38 & 0.00 & 0.62 & 0.00 & 0.03 & 2.79 & \\
\hline $\mathrm{OX}(\mathrm{wt} \%)$ & 0.08 & 0.05 & 17.95 & 14.67 & 0.00 & 1.70 & 17.42 & 39.80 & 0.00 & 0.62 & 0.00 & 0.07 & 2.79 & \\
\hline TMBen (wt $\%)$ & 0.00 & 0.00 & 0.00 & 0.00 & 0.00 & 0.00 & 0.00 & 0.00 & 0.00 & 0.00 & 0.00 & 0.00 & 0.00 & \\
\hline PDEB (wt\%) & 99.92 & 99.92 & 0.15 & 0.15 & 0.00 & 0.05 & 0.12 & 1.18 & 0.00 & 0.00 & 0.00 & 0.13 & 0.00 & \\
\hline
\end{tabular}


Table S47 Mass balance for the modified aromatics complex with toluene methylation and crystallization at 218 K after step 4 (E4)

\begin{tabular}{|c|c|c|c|c|c|c|c|c|c|c|c|c|c|c|}
\hline Stream & 1 & 2 & 3 & 4 & 5 & 6 & 7 & 8 & 9 & 10 & 11 & 12 & 13 & 14 \\
\hline Flow $(\mathrm{t} / \mathrm{h})$ & 38.10 & 20.60 & 17.50 & 68.96 & 61.58 & 18.70 & 63.94 & 7.37 & 4.89 & 60.36 & 27.34 & 1.20 & 6.18 & 7.22 \\
\hline $\operatorname{Ben}(w t \%)$ & 12.86 & 23.78 & 0.00 & 0.00 & 0.00 & 0.00 & 0.00 & 0.00 & 99.88 & 0.11 & 0.00 & 0.00 & 0.00 & 0.00 \\
\hline Tol (wt\%) & 41.21 & 76.22 & 0.00 & 0.02 & 0.02 & 0.00 & 0.00 & 0.00 & 0.12 & 99.78 & 0.00 & 0.00 & 0.00 & 0.00 \\
\hline $\mathrm{ECH}(\mathrm{wt} \%)$ & 0.00 & 0.00 & 0.00 & 18.68 & 20.91 & 0.45 & 20.02 & 0.00 & 0.00 & 0.00 & 0.00 & 0.00 & 0.00 & 0.00 \\
\hline $\mathrm{EB}(\mathrm{wt} \%)$ & 3.20 & 0.00 & 6.97 & 6.29 & 7.05 & 0.02 & 6.79 & 0.00 & 0.00 & 0.00 & 0.00 & 0.00 & 0.00 & 0.00 \\
\hline $\mathrm{PX}(\mathrm{wt} \%)$ & 6.56 & 0.00 & 14.28 & 16.45 & 18.42 & 60.65 & 0.02 & 0.00 & 0.00 & 0.12 & 100.00 & 0.00 & 0.00 & 0.08 \\
\hline $\mathrm{MX}(\mathrm{wt} \%)$ & 13.88 & 0.00 & 30.22 & 35.18 & 39.38 & 0.13 & 37.90 & 0.09 & 0.00 & 0.00 & 0.00 & 0.56 & 0.00 & 0.00 \\
\hline $\mathrm{OX}(\mathrm{wt} \%)$ & 6.29 & 0.00 & 13.69 & 14.43 & 14.21 & 0.07 & 13.69 & 16.19 & 0.00 & 0.00 & 0.00 & 99.33 & 0.09 & 0.00 \\
\hline TMBen (wt\%) & 16.00 & 0.00 & 34.84 & 8.84 & 0.00 & 0.00 & 0.00 & 82.67 & 0.00 & 0.00 & 0.00 & 0.11 & 98.66 & 0.00 \\
\hline PDEB (wt\%) & 0.00 & 0.00 & 0.00 & 0.11 & 0.00 & 38.69 & 21.59 & 1.05 & 0.00 & 0.00 & 0.00 & 0.00 & 1.25 & 99.92 \\
\hline Stream & 15 & 16 & 17 & 18 & 19 & 20 & 21 & 22 & 23 & 24 & 25 & 26 & 27 & \\
\hline Flow $(\mathrm{t} / \mathrm{h})$ & 13.75 & 20.98 & 51.46 & 51.46 & 20.60 & 28.62 & 50.19 & 1.27 & 76.07 & 77.51 & 15.71 & 11.47 & 17.15 & \\
\hline Ben $(w t \%)$ & 0.00 & 0.00 & 0.00 & 0.00 & 23.78 & 0.00 & 0.00 & 0.00 & 0.10 & 0.08 & 0.08 & 0.00 & 0.00 & \\
\hline Tol (wt\%) & 0.00 & 0.00 & 0.03 & 0.03 & 76.22 & 0.05 & 0.00 & 1.17 & 99.81 & 77.73 & 99.92 & 0.00 & 0.09 & \\
\hline $\mathrm{ECH}(\mathrm{wt} \%)$ & 0.00 & 0.00 & 25.04 & 25.03 & 0.00 & 0.29 & 25.50 & 6.62 & 0.00 & 0.00 & 0.00 & 0.73 & 0.00 & \\
\hline $\mathrm{EB}(w \mathrm{t} \%)$ & 0.00 & 0.00 & 8.44 & 6.07 & 0.00 & 0.01 & 8.65 & 0.25 & 0.00 & 0.00 & 0.00 & 0.03 & 0.00 & \\
\hline $\mathrm{PX}(\mathrm{wt} \%)$ & 0.00 & 0.03 & 0.34 & 17.19 & 0.00 & 96.12 & 0.02 & 12.86 & 0.09 & 20.96 & 0.00 & 98.79 & 94.34 & \\
\hline MX (wt\%) & 0.00 & 0.00 & 48.06 & 36.86 & 0.00 & 1.75 & 48.28 & 39.41 & 0.00 & 0.62 & 0.00 & 0.21 & 2.79 & \\
\hline $\mathrm{OX}(w \mathrm{t} \%)$ & 0.08 & 0.05 & 17.94 & 14.67 & 0.00 & 1.72 & 17.42 & 38.56 & 0.00 & 0.62 & 0.00 & 0.11 & 2.79 & \\
\hline TMBen (wt $\%)$ & 0.00 & 0.00 & 0.00 & 0.00 & 0.00 & 0.00 & 0.00 & 0.00 & 0.00 & 0.00 & 0.00 & 0.00 & 0.00 & \\
\hline PDEB (wt\%) & 99.92 & 99.92 & 0.15 & 0.15 & 0.00 & 0.05 & 0.12 & 1.14 & 0.00 & 0.00 & 0.00 & 0.13 & 0.00 & \\
\hline
\end{tabular}


Table S48 Mass balance for the modified aromatics complex with toluene methylation and crystallization at $218 \mathrm{~K}$ after step 5 (E5)

\begin{tabular}{|c|c|c|c|c|c|c|c|c|c|c|c|c|c|c|}
\hline Stream & 1 & 2 & 3 & 4 & 5 & 6 & 7 & 8 & 9 & 10 & 11 & 12 & 13 & 14 \\
\hline Flow $(\mathrm{t} / \mathrm{h})$ & 38.10 & 20.60 & 17.50 & 68.88 & 61.50 & 18.13 & 63.54 & 7.37 & 4.89 & 60.36 & 27.35 & 1.19 & 6.18 & 6.72 \\
\hline $\operatorname{Ben}(w t \%)$ & 12.86 & 23.78 & 0.00 & 0.00 & 0.00 & 0.00 & 0.00 & 0.00 & 99.88 & 0.11 & 0.00 & 0.00 & 0.00 & 0.00 \\
\hline Tol (wt\%) & 41.21 & 76.22 & 0.00 & 0.02 & 0.02 & 0.00 & 0.00 & 0.00 & 0.12 & 99.78 & 0.00 & 0.00 & 0.00 & 0.00 \\
\hline $\mathrm{ECH}(\mathrm{wt} \%)$ & 0.00 & 0.00 & 0.00 & 18.67 & 20.91 & 0.30 & 20.16 & 0.00 & 0.00 & 0.00 & 0.00 & 0.00 & 0.00 & 0.00 \\
\hline EB (wt\%) & 3.20 & 0.00 & 6.97 & 6.30 & 7.05 & 0.02 & 6.82 & 0.00 & 0.00 & 0.00 & 0.00 & 0.00 & 0.00 & 0.00 \\
\hline $\mathrm{PX}(\mathrm{wt} \%)$ & 6.56 & 0.00 & 14.28 & 16.45 & 18.42 & 62.51 & 0.01 & 0.00 & 0.00 & 0.12 & 100.00 & 0.00 & 0.00 & 0.08 \\
\hline $\mathrm{MX}(w \mathrm{t} \%)$ & 13.88 & 0.00 & 30.22 & 35.17 & 39.38 & 0.01 & 38.13 & 0.09 & 0.00 & 0.00 & 0.00 & 0.56 & 0.00 & 0.00 \\
\hline $\mathrm{OX}(\mathrm{wt} \%)$ & 6.29 & 0.00 & 13.69 & 14.43 & 14.22 & 0.05 & 13.77 & 16.18 & 0.00 & 0.00 & 0.00 & 99.33 & 0.09 & 0.00 \\
\hline TMBen (wt\%) & 16.00 & 0.00 & 34.84 & 8.85 & 0.00 & 0.00 & 0.00 & 82.69 & 0.00 & 0.00 & 0.00 & 0.11 & 98.67 & 0.00 \\
\hline PDEB (wt\%) & 0.00 & 0.00 & 0.00 & 0.11 & 0.00 & 37.12 & 21.12 & 1.04 & 0.00 & 0.00 & 0.00 & 0.00 & 1.25 & 99.92 \\
\hline Stream & 15 & 16 & 17 & 18 & 19 & 20 & 21 & 22 & 23 & 24 & 25 & 26 & 27 & \\
\hline Flow $(\mathrm{t} / \mathrm{h})$ & 13.36 & 20.08 & 51.38 & 51.38 & 20.60 & 28.55 & 50.17 & 1.21 & 76.07 & 77.51 & 15.71 & 11.41 & 17.15 & \\
\hline Ben (wt $\%)$ & 0.00 & 0.00 & 0.00 & 0.00 & 23.78 & 0.00 & 0.00 & 0.00 & 0.10 & 0.08 & 0.08 & 0.00 & 0.00 & \\
\hline Tol (wt\%) & 0.00 & 0.00 & 0.03 & 0.03 & 76.22 & 0.05 & 0.00 & 1.23 & 99.81 & 77.73 & 99.92 & 0.00 & 0.09 & \\
\hline $\mathrm{ECH}(\mathrm{wt} \%)$ & 0.00 & 0.00 & 25.04 & 25.03 & 0.00 & 0.19 & 25.53 & 4.50 & 0.00 & 0.00 & 0.00 & 0.48 & 0.00 & \\
\hline EB (wt\%) & 0.00 & 0.00 & 8.44 & 6.07 & 0.00 & 0.01 & 8.64 & 0.27 & 0.00 & 0.00 & 0.00 & 0.03 & 0.00 & \\
\hline $\mathrm{PX}(\mathrm{wt} \%)$ & 0.00 & 0.03 & 0.31 & 17.19 & 0.00 & 96.32 & 0.01 & 12.88 & 0.09 & 20.96 & 0.00 & 99.29 & 94.34 & \\
\hline MX (wt\%) & 0.00 & 0.00 & 48.08 & 36.86 & 0.00 & 1.68 & 48.28 & 39.66 & 0.00 & 0.62 & 0.00 & 0.01 & 2.79 & \\
\hline OX $(w t \%)$ & 0.08 & 0.05 & 17.95 & 14.67 & 0.00 & 1.70 & 17.41 & 40.27 & 0.00 & 0.62 & 0.00 & 0.07 & 2.79 & \\
\hline TMBen (wt\%) & 0.00 & 0.00 & 0.00 & 0.00 & 0.00 & 0.00 & 0.00 & 0.00 & 0.00 & 0.00 & 0.00 & 0.00 & 0.00 & \\
\hline PDEB (wt\%) & 99.92 & 99.92 & 0.15 & 0.15 & 0.00 & 0.05 & 0.12 & 1.19 & 0.00 & 0.00 & 0.00 & 0.13 & 0.00 & \\
\hline
\end{tabular}




\section{S12. Case F results}

Table S49 Optimization of the modified aromatics complex with toluene methylation and crystallization at $244 \mathrm{~K}(\mathrm{~F})$

\begin{tabular}{|c|c|c|c|c|c|}
\hline & Step 1 & Step 2 & Step 3 & Step 4 & Step 5 \\
\hline Profit $\left(\$ / t_{\text {Reformate }}\right)$ & 1041.7 & 1043.6 & 1047.2 & 1047.1 & 1047.2 \\
\hline$\Delta$ Revenue $\times 10^{-3}(\$ / \text { year })^{\mathrm{a}}$ & 26339 & 26920 & 28002 & 27964 & 28007 \\
\hline \multicolumn{6}{|l|}{ Operation Cost $(\$ / \mathrm{h})$ : } \\
\hline Aromatics Extraction & 353.6 & 353.6 & 353.6 & 353.6 & 353.6 \\
\hline Benzene Column & 142.8 & 142.8 & 142.8 & 142.8 & 142.8 \\
\hline Xylene Splitter & 317.4 & 315.3 & 314.0 & 314.0 & 313.9 \\
\hline Extract Column & 220.4 & 200.2 & 127.0 & 129.4 & 127.0 \\
\hline Raffinate Column & 539.1 & 526.6 & 490.4 & 491.5 & 490.3 \\
\hline Isomerization & 400.4 & 397.6 & 395.1 & 395.1 & 395.0 \\
\hline$o$-Xylene Column & 62.4 & 62.6 & 62.5 & 62.5 & 62.5 \\
\hline Crystallization & 167.6 & 166.5 & 163.7 & 164.0 & 163.7 \\
\hline Toluene Methylation & 1085.8 & 1073.6 & 1074.0 & 1074.7 & 1074.0 \\
\hline Toluene Column & 846.8 & 862.5 & 861.9 & 862.5 & 861.9 \\
\hline \multicolumn{6}{|l|}{ SMB: } \\
\hline Configuration & $5-9-7-3$ & $10-5-3-6$ & $10-5-3-6$ & $5-9-7-3$ & $9-4-4-7$ \\
\hline Switching time (s) & 66 & 66 & 180 & 180 & 180 \\
\hline Productivity $\left(\mathrm{kg} / \mathrm{hm}^{3}\right)$ & 54.32 & 53.72 & 52.53 & 52.63 & 52.53 \\
\hline Desorbent consumption $(\mathrm{kg} / \mathrm{kg})$ & 5.01 & 4.34 & 1.70 & 1.77 & 1.70 \\
\hline Recovery $(\%)$ & 98.92 & 99.40 & 99.93 & 99.90 & 99.96 \\
\hline Total $\Delta \mathrm{P}(\mathrm{kPa})$ & 229 & 228 & 68 & 71 & 68 \\
\hline Maximum $\Delta \mathrm{P} /$ bed $(\mathrm{kPa})$ & 10.6 & 10.6 & 3.8 & 3.8 & 3.8 \\
\hline \multicolumn{6}{|l|}{ Toluene Methylation: } \\
\hline Toluene conversion $(\%)$ & 20.89 & 20.56 & 20.57 & 20.56 & 20.57 \\
\hline$p$-Xylene selectivity (\%) & 94.23 & 94.42 & 94.40 & 94.42 & 94.40 \\
\hline Toluene/MeOH (mol/mol) & 1.64 & 1.70 & 1.70 & 1.70 & 1.70 \\
\hline Water/Hydrocarbons (mol/mol) & 0.31 & 0.29 & 0.29 & 0.29 & 0.29 \\
\hline Space time $\left(\mathrm{g}_{\text {cat }} \mathrm{h} / \mathrm{mol}_{\text {Tol }}\right)$ & 3.72 & 3.50 & 3.51 & 3.50 & 3.51 \\
\hline $\mathrm{MeOH}$ loss $(\%)$ & 36.71 & 36.78 & 36.73 & 36.78 & 36.73 \\
\hline
\end{tabular}

a Compared to the base case (A0) assuming 330 days of operation per year. 
Table S50 Mass balance for the modified aromatics complex with toluene methylation and crystallization at $244 \mathrm{~K}$ after step 1 (F1)

\begin{tabular}{|c|c|c|c|c|c|c|c|c|c|c|c|c|c|c|}
\hline Stream & 1 & 2 & 3 & 4 & 5 & 6 & 7 & 8 & 9 & 10 & 11 & 12 & 13 & 14 \\
\hline Flow $(\mathrm{t} / \mathrm{h})$ & 38.10 & 20.60 & 17.50 & 68.87 & 64.33 & 47.12 & 77.83 & 7.36 & 4.89 & 59.11 & 27.33 & 1.20 & 6.16 & 34.10 \\
\hline $\operatorname{Ben}(w t \%)$ & 12.86 & 23.78 & 0.00 & 0.00 & 0.00 & 0.00 & 0.00 & 0.00 & 99.88 & 0.11 & 0.00 & 0.00 & 0.00 & 0.00 \\
\hline Tol (wt\%) & 41.21 & 76.22 & 0.00 & 0.00 & 0.02 & 0.00 & 0.00 & 0.00 & 0.12 & 99.78 & 0.00 & 0.00 & 0.00 & 0.00 \\
\hline $\mathrm{ECH}(\mathrm{wt} \%)$ & 0.00 & 0.00 & 0.00 & 18.67 & 20.58 & 0.81 & 16.52 & 0.00 & 0.00 & 0.00 & 0.00 & 0.00 & 0.00 & 0.00 \\
\hline $\mathrm{EB}(\mathrm{wt} \%)$ & 3.20 & 0.00 & 6.97 & 6.30 & 6.81 & 0.09 & 5.57 & 0.00 & 0.00 & 0.00 & 0.00 & 0.00 & 0.00 & 0.00 \\
\hline $\mathrm{PX}(\mathrm{wt} \%)$ & 6.56 & 0.00 & 14.28 & 16.46 & 18.95 & 25.65 & 0.17 & 0.00 & 0.00 & 0.12 & 100.00 & 0.00 & 0.00 & 0.08 \\
\hline $\mathrm{MX}(\mathrm{wt} \%)$ & 13.88 & 0.00 & 30.22 & 35.19 & 39.03 & 0.81 & 31.77 & 0.09 & 0.00 & 0.00 & 0.00 & 0.55 & 0.00 & 0.00 \\
\hline $\mathrm{OX}(\mathrm{wt} \%)$ & 6.29 & 0.00 & 13.69 & 14.43 & 14.58 & 0.29 & 11.90 & 16.21 & 0.00 & 0.00 & 0.00 & 99.33 & 0.09 & 0.00 \\
\hline TMBen (wt\%) & 16.00 & 0.00 & 34.84 & 8.85 & 0.00 & 0.00 & 0.00 & 82.83 & 0.00 & 0.00 & 0.00 & 0.11 & 98.87 & 0.00 \\
\hline PDEB (wt\%) & 0.00 & 0.00 & 0.00 & 0.09 & 0.03 & 72.34 & 34.06 & 0.87 & 0.00 & 0.00 & 0.00 & 0.00 & 1.04 & 99.92 \\
\hline Stream & 15 & 16 & 17 & 18 & 19 & 20 & 21 & 22 & 23 & 24 & 25 & 26 & 27 & \\
\hline Flow $(\mathrm{t} / \mathrm{h})$ & 26.46 & 60.56 & 51.37 & 51.37 & 20.60 & 30.16 & 61.51 & 2.83 & 74.81 & 76.24 & 15.71 & 13.02 & 17.13 & \\
\hline Ben $(w t \%)$ & 0.00 & 0.00 & 0.00 & 0.00 & 23.78 & 0.00 & 0.00 & 0.00 & 0.10 & 0.08 & 0.08 & 0.00 & 0.00 & \\
\hline Tol (wt\%) & 0.00 & 0.00 & 0.00 & 0.00 & 76.22 & 0.05 & 0.00 & 0.53 & 99.81 & 77.38 & 99.92 & 0.00 & 0.09 & \\
\hline $\mathrm{ECH}(\mathrm{wt} \%)$ & 0.00 & 0.00 & 25.03 & 25.03 & 0.00 & 1.27 & 20.90 & 13.56 & 0.00 & 0.00 & 0.00 & 2.94 & 0.00 & \\
\hline $\mathrm{EB}(w \mathrm{t} \%)$ & 0.00 & 0.00 & 8.45 & 6.07 & 0.00 & 0.14 & 7.05 & 1.54 & 0.00 & 0.00 & 0.00 & 0.33 & 0.00 & \\
\hline $\mathrm{PX}(\mathrm{wt} \%)$ & 0.00 & 0.04 & 0.26 & 17.20 & 0.00 & 93.47 & 18.43 & 30.32 & 0.09 & 21.24 & 0.00 & 92.60 & 94.13 & \\
\hline MX (wt\%) & 0.00 & 0.00 & 48.14 & 36.89 & 0.00 & 2.92 & 39.39 & 31.11 & 0.00 & 0.65 & 0.00 & 2.95 & 2.89 & \\
\hline $\mathrm{OX}(w \mathrm{t} \%)$ & 0.08 & 0.03 & 18.00 & 14.68 & 0.00 & 2.09 & 14.22 & 22.35 & 0.00 & 0.65 & 0.00 & 1.05 & 2.89 & \\
\hline TMBen (wt\%) & 0.00 & 0.00 & 0.00 & 0.00 & 0.00 & 0.00 & 0.00 & 0.00 & 0.00 & 0.00 & 0.00 & 0.00 & 0.00 & \\
\hline PDEB (wt\%) & 99.92 & 99.92 & 0.12 & 0.12 & 0.00 & 0.05 & 0.00 & 0.58 & 0.00 & 0.00 & 0.00 & 0.13 & 0.00 & \\
\hline
\end{tabular}


Table S51 Mass balance for the modified aromatics complex with toluene methylation and crystallization at $244 \mathrm{~K}$ after step 2 (F2)

\begin{tabular}{|c|c|c|c|c|c|c|c|c|c|c|c|c|c|c|}
\hline Stream & 1 & 2 & 3 & 4 & 5 & 6 & 7 & 8 & 9 & 10 & 11 & 12 & 13 & 14 \\
\hline Flow $(\mathrm{t} / \mathrm{h})$ & 38.10 & 20.60 & 17.50 & 68.38 & 63.51 & 40.74 & 74.68 & 7.35 & 4.89 & 60.32 & 27.35 & 1.19 & 6.16 & 28.06 \\
\hline $\operatorname{Ben}(w t \%)$ & 12.86 & 23.78 & 0.00 & 0.00 & 0.00 & 0.00 & 0.00 & 0.00 & 99.88 & 0.11 & 0.00 & 0.00 & 0.00 & 0.00 \\
\hline Tol (wt\%) & 41.21 & 76.22 & 0.00 & 0.00 & 0.02 & 0.00 & 0.00 & 0.00 & 0.12 & 99.78 & 0.00 & 0.00 & 0.00 & 0.00 \\
\hline $\mathrm{ECH}(\mathrm{wt} \%)$ & 0.00 & 0.00 & 0.00 & 18.63 & 20.61 & 0.86 & 17.06 & 0.00 & 0.00 & 0.00 & 0.00 & 0.00 & 0.00 & 0.00 \\
\hline $\mathrm{EB}(\mathrm{wt} \%)$ & 3.20 & 0.00 & 6.97 & 6.30 & 6.86 & 0.13 & 5.77 & 0.00 & 0.00 & 0.00 & 0.00 & 0.00 & 0.00 & 0.00 \\
\hline $\mathrm{PX}(\mathrm{wt} \%)$ & 6.56 & 0.00 & 14.28 & 16.46 & 18.90 & 29.34 & 0.10 & 0.00 & 0.00 & 0.12 & 100.00 & 0.00 & 0.00 & 0.08 \\
\hline $\mathrm{MX}(\mathrm{wt} \%)$ & 13.88 & 0.00 & 30.22 & 35.18 & 39.00 & 0.59 & 32.85 & 0.09 & 0.00 & 0.00 & 0.00 & 0.56 & 0.00 & 0.00 \\
\hline $\mathrm{OX}(\mathrm{wt} \%)$ & 6.29 & 0.00 & 13.69 & 14.43 & 14.57 & 0.23 & 12.30 & 16.11 & 0.00 & 0.00 & 0.00 & 99.33 & 0.09 & 0.00 \\
\hline TMBen (wt\%) & 16.00 & 0.00 & 34.84 & 8.91 & 0.00 & 0.00 & 0.00 & 82.93 & 0.00 & 0.00 & 0.00 & 0.11 & 98.88 & 0.00 \\
\hline PDEB (wt\%) & 0.00 & 0.00 & 0.00 & 0.09 & 0.03 & 68.85 & 31.92 & 0.86 & 0.00 & 0.00 & 0.00 & 0.00 & 1.03 & 99.92 \\
\hline Stream & 15 & 16 & 17 & 18 & 19 & 20 & 21 & 22 & 23 & 24 & 25 & 26 & 27 & \\
\hline Flow $(\mathrm{t} / \mathrm{h})$ & 23.79 & 51.85 & 50.88 & 50.88 & 20.60 & 29.83 & 61.03 & 2.48 & 76.02 & 77.46 & 15.71 & 12.68 & 17.14 & \\
\hline $\operatorname{Ben}(w t \%)$ & 0.00 & 0.00 & 0.00 & 0.00 & 23.78 & 0.00 & 0.00 & 0.00 & 0.10 & 0.08 & 0.08 & 0.00 & 0.00 & \\
\hline Tol (wt\%) & 0.00 & 0.00 & 0.00 & 0.00 & 76.22 & 0.05 & 0.00 & 0.60 & 99.81 & 77.71 & 99.92 & 0.00 & 0.09 & \\
\hline $\mathrm{ECH}(\mathrm{wt} \%)$ & 0.00 & 0.00 & 25.03 & 25.03 & 0.00 & 1.18 & 20.87 & 14.21 & 0.00 & 0.00 & 0.00 & 2.78 & 0.00 & \\
\hline $\mathrm{EB}(w \mathrm{t} \%)$ & 0.00 & 0.00 & 8.47 & 6.07 & 0.00 & 0.17 & 7.06 & 2.09 & 0.00 & 0.00 & 0.00 & 0.41 & 0.00 & \\
\hline $\mathrm{PX}(\mathrm{wt} \%)$ & 0.00 & 0.04 & 0.14 & 17.20 & 0.00 & 94.21 & 18.44 & 30.29 & 0.09 & 20.97 & 0.00 & 94.05 & 94.32 & \\
\hline MX (wt\%) & 0.00 & 0.00 & 48.22 & 36.89 & 0.00 & 2.42 & 39.41 & 29.08 & 0.00 & 0.62 & 0.00 & 1.90 & 2.80 & \\
\hline $\mathrm{OX}(w \mathrm{t} \%)$ & 0.08 & 0.04 & 18.01 & 14.68 & 0.00 & 1.92 & 14.23 & 23.07 & 0.00 & 0.62 & 0.00 & 0.73 & 2.80 & \\
\hline TMBen (wt $\%)$ & 0.00 & 0.00 & 0.00 & 0.00 & 0.00 & 0.00 & 0.00 & 0.00 & 0.00 & 0.00 & 0.00 & 0.00 & 0.00 & \\
\hline PDEB (wt\%) & 99.92 & 99.92 & 0.12 & 0.12 & 0.00 & 0.05 & 0.00 & 0.65 & 0.00 & 0.00 & 0.00 & 0.13 & 0.00 & \\
\hline
\end{tabular}


Table S52 Mass balance for the modified aromatics complex with toluene methylation and crystallization at $244 \mathrm{~K}$ after step 3 (F3)

\begin{tabular}{|c|c|c|c|c|c|c|c|c|c|c|c|c|c|c|}
\hline Stream & 1 & 2 & 3 & 4 & 5 & 6 & 7 & 8 & 9 & 10 & 11 & 12 & 13 & 14 \\
\hline Flow $(\mathrm{t} / \mathrm{h})$ & 38.10 & 20.60 & 17.50 & 68.06 & 62.34 & 18.40 & 63.87 & 7.34 & 4.89 & 60.27 & 27.36 & 1.18 & 6.16 & 6.57 \\
\hline $\operatorname{Ben}(w t \%)$ & 12.86 & 23.78 & 0.00 & 0.00 & 0.00 & 0.00 & 0.00 & 0.00 & 99.88 & 0.11 & 0.00 & 0.00 & 0.00 & 0.00 \\
\hline Tol (wt\%) & 41.21 & 76.22 & 0.00 & 0.00 & 0.02 & 0.00 & 0.00 & 0.00 & 0.12 & 99.78 & 0.00 & 0.00 & 0.00 & 0.00 \\
\hline $\mathrm{ECH}(\mathrm{wt} \%)$ & 0.00 & 0.00 & 0.00 & 18.60 & 20.49 & 0.61 & 19.82 & 0.00 & 0.00 & 0.00 & 0.00 & 0.00 & 0.00 & 0.00 \\
\hline $\mathrm{EB}(\mathrm{wt} \%)$ & 3.20 & 0.00 & 6.97 & 6.30 & 6.88 & 0.01 & 6.72 & 0.00 & 0.00 & 0.00 & 0.00 & 0.00 & 0.00 & 0.00 \\
\hline $\mathrm{PX}(\mathrm{wt} \%)$ & 6.56 & 0.00 & 14.28 & 16.45 & 18.75 & 63.51 & 0.01 & 0.00 & 0.00 & 0.12 & 100.00 & 0.00 & 0.00 & 0.08 \\
\hline $\mathrm{MX}(\mathrm{wt} \%)$ & 13.88 & 0.00 & 30.22 & 35.17 & 39.18 & 0.04 & 38.24 & 0.09 & 0.00 & 0.00 & 0.00 & 0.56 & 0.00 & 0.00 \\
\hline $\mathrm{OX}(\mathrm{wt} \%)$ & 6.29 & 0.00 & 13.69 & 14.43 & 14.65 & 0.05 & 14.31 & 16.05 & 0.00 & 0.00 & 0.00 & 99.33 & 0.09 & 0.00 \\
\hline TMBen (wt\%) & 16.00 & 0.00 & 34.84 & 8.96 & 0.00 & 0.00 & 0.00 & 83.00 & 0.00 & 0.00 & 0.00 & 0.11 & 98.89 & 0.00 \\
\hline PDEB (wt\%) & 0.00 & 0.00 & 0.00 & 0.09 & 0.02 & 35.78 & 20.91 & 0.86 & 0.00 & 0.00 & 0.00 & 0.00 & 1.02 & 99.92 \\
\hline Stream & 15 & 16 & 17 & 18 & 19 & 20 & 21 & 22 & 23 & 24 & 25 & 26 & 27 & \\
\hline Flow $(\mathrm{t} / \mathrm{h})$ & 13.30 & 19.87 & 50.57 & 50.57 & 20.60 & 28.97 & 60.72 & 1.61 & 75.97 & 77.41 & 15.71 & 11.83 & 17.14 & \\
\hline Ben $(w t \%)$ & 0.00 & 0.00 & 0.00 & 0.00 & 23.78 & 0.00 & 0.00 & 0.00 & 0.10 & 0.08 & 0.08 & 0.00 & 0.00 & \\
\hline Tol (wt\%) & 0.00 & 0.00 & 0.00 & 0.00 & 76.22 & 0.05 & 0.00 & 0.92 & 99.81 & 77.70 & 99.92 & 0.00 & 0.09 & \\
\hline $\mathrm{ECH}(\mathrm{wt} \%)$ & 0.00 & 0.00 & 25.04 & 25.03 & 0.00 & 0.39 & 20.84 & 6.99 & 0.00 & 0.00 & 0.00 & 0.95 & 0.00 & \\
\hline $\mathrm{EB}(w \mathrm{t} \%)$ & 0.00 & 0.00 & 8.48 & 6.07 & 0.00 & 0.00 & 7.06 & 0.09 & 0.00 & 0.00 & 0.00 & 0.01 & 0.00 & \\
\hline $\mathrm{PX}(\mathrm{wt} \%)$ & 0.00 & 0.03 & 0.02 & 17.20 & 0.00 & 96.13 & 18.44 & 30.53 & 0.09 & 20.97 & 0.00 & 98.77 & 94.30 & \\
\hline MX (wt\%) & 0.00 & 0.00 & 48.29 & 36.89 & 0.00 & 1.68 & 39.42 & 30.22 & 0.00 & 0.62 & 0.00 & 0.06 & 2.80 & \\
\hline $\mathrm{OX}(\mathrm{wt} \%)$ & 0.08 & 0.05 & 18.05 & 14.68 & 0.00 & 1.69 & 14.23 & 30.33 & 0.00 & 0.62 & 0.00 & 0.08 & 2.80 & \\
\hline TMBen (wt $\%)$ & 0.00 & 0.00 & 0.00 & 0.00 & 0.00 & 0.00 & 0.00 & 0.00 & 0.00 & 0.00 & 0.00 & 0.00 & 0.00 & \\
\hline PDEB (wt\%) & 99.92 & 99.92 & 0.12 & 0.12 & 0.00 & 0.05 & 0.00 & 0.93 & 0.00 & 0.00 & 0.00 & 0.13 & 0.00 & \\
\hline
\end{tabular}


Table S53 Mass balance for the modified aromatics complex with toluene methylation and crystallization at $244 \mathrm{~K}$ after step 4 (F4)

\begin{tabular}{|c|c|c|c|c|c|c|c|c|c|c|c|c|c|c|}
\hline Stream & 1 & 2 & 3 & 4 & 5 & 6 & 7 & 8 & 9 & 10 & 11 & 12 & 13 & 14 \\
\hline Flow $(\mathrm{t} / \mathrm{h})$ & 38.10 & 20.60 & 17.50 & 68.07 & 62.42 & 19.00 & 64.19 & 7.34 & 4.89 & 60.32 & 27.36 & 1.18 & 6.16 & 7.09 \\
\hline $\operatorname{Ben}(w t \%)$ & 12.86 & 23.78 & 0.00 & 0.00 & 0.00 & 0.00 & 0.00 & 0.00 & 99.88 & 0.11 & 0.00 & 0.00 & 0.00 & 0.00 \\
\hline Tol (wt\%) & 41.21 & 76.22 & 0.00 & 0.00 & 0.02 & 0.00 & 0.00 & 0.00 & 0.12 & 99.78 & 0.00 & 0.00 & 0.00 & 0.00 \\
\hline $\mathrm{ECH}(\mathrm{wt} \%)$ & 0.00 & 0.00 & 0.00 & 18.60 & 20.49 & 0.69 & 19.72 & 0.00 & 0.00 & 0.00 & 0.00 & 0.00 & 0.00 & 0.00 \\
\hline $\mathrm{EB}(\mathrm{wt} \%)$ & 3.20 & 0.00 & 6.97 & 6.30 & 6.87 & 0.01 & 6.68 & 0.00 & 0.00 & 0.00 & 0.00 & 0.00 & 0.00 & 0.00 \\
\hline $\mathrm{PX}(\mathrm{wt} \%)$ & 6.56 & 0.00 & 14.28 & 16.45 & 18.77 & 61.63 & 0.02 & 0.00 & 0.00 & 0.12 & 100.00 & 0.00 & 0.00 & 0.08 \\
\hline $\mathrm{MX}(\mathrm{wt} \%)$ & 13.88 & 0.00 & 30.22 & 35.17 & 39.18 & 0.22 & 38.04 & 0.09 & 0.00 & 0.00 & 0.00 & 0.56 & 0.00 & 0.00 \\
\hline $\mathrm{OX}(\mathrm{wt} \%)$ & 6.29 & 0.00 & 13.69 & 14.43 & 14.64 & 0.09 & 14.23 & 16.05 & 0.00 & 0.00 & 0.00 & 99.33 & 0.09 & 0.00 \\
\hline TMBen (wt\%) & 16.00 & 0.00 & 34.84 & 8.95 & 0.00 & 0.00 & 0.00 & 83.00 & 0.00 & 0.00 & 0.00 & 0.11 & 98.89 & 0.00 \\
\hline PDEB (wt\%) & 0.00 & 0.00 & 0.00 & 0.09 & 0.02 & 37.36 & 21.30 & 0.86 & 0.00 & 0.00 & 0.00 & 0.00 & 1.02 & 99.92 \\
\hline Stream & 15 & 16 & 17 & 18 & 19 & 20 & 21 & 22 & 23 & 24 & 25 & 26 & 27 & \\
\hline Flow $(\mathrm{t} / \mathrm{h})$ & 13.62 & 20.71 & 50.57 & 50.57 & 20.60 & 29.06 & 60.72 & 1.70 & 76.02 & 77.46 & 15.71 & 11.91 & 17.14 & \\
\hline Ben $(w t \%)$ & 0.00 & 0.00 & 0.00 & 0.00 & 23.78 & 0.00 & 0.00 & 0.00 & 0.10 & 0.08 & 0.08 & 0.00 & 0.00 & \\
\hline Tol (wt\%) & 0.00 & 0.00 & 0.00 & 0.00 & 76.22 & 0.05 & 0.00 & 0.88 & 99.81 & 77.71 & 99.92 & 0.00 & 0.09 & \\
\hline $\mathrm{ECH}(\mathrm{wt} \%)$ & 0.00 & 0.00 & 25.04 & 25.03 & 0.00 & 0.45 & 20.84 & 7.75 & 0.00 & 0.00 & 0.00 & 1.11 & 0.00 & \\
\hline $\mathrm{EB}(w \mathrm{t} \%)$ & 0.00 & 0.00 & 8.48 & 6.07 & 0.00 & 0.00 & 7.06 & 0.08 & 0.00 & 0.00 & 0.00 & 0.01 & 0.00 & \\
\hline $\mathrm{PX}(\mathrm{wt} \%)$ & 0.00 & 0.03 & 0.02 & 17.20 & 0.00 & 95.94 & 18.44 & 30.51 & 0.09 & 20.97 & 0.00 & 98.26 & 94.32 & \\
\hline MX (wt\%) & 0.00 & 0.00 & 48.29 & 36.89 & 0.00 & 1.79 & 39.42 & 30.67 & 0.00 & 0.62 & 0.00 & 0.35 & 2.80 & \\
\hline $\mathrm{OX}(w \mathrm{t} \%)$ & 0.08 & 0.05 & 18.04 & 14.68 & 0.00 & 1.71 & 14.23 & 29.24 & 0.00 & 0.62 & 0.00 & 0.15 & 2.80 & \\
\hline TMBen (wt $\%)$ & 0.00 & 0.00 & 0.00 & 0.00 & 0.00 & 0.00 & 0.00 & 0.00 & 0.00 & 0.00 & 0.00 & 0.00 & 0.00 & \\
\hline PDEB (wt\%) & 99.92 & 99.92 & 0.12 & 0.12 & 0.00 & 0.05 & 0.00 & 0.89 & 0.00 & 0.00 & 0.00 & 0.13 & 0.00 & \\
\hline
\end{tabular}


Table S54 Mass balance for the modified aromatics complex with toluene methylation and crystallization at $244 \mathrm{~K}$ after step 5 (F5)

\begin{tabular}{|c|c|c|c|c|c|c|c|c|c|c|c|c|c|c|}
\hline Stream & 1 & 2 & 3 & 4 & 5 & 6 & 7 & 8 & 9 & 10 & 11 & 12 & 13 & 14 \\
\hline Flow $(\mathrm{t} / \mathrm{h})$ & 38.10 & 20.60 & 17.50 & 68.05 & 62.32 & 18.39 & 63.85 & 7.34 & 4.89 & 60.27 & 27.36 & 1.18 & 6.16 & 6.56 \\
\hline $\operatorname{Ben}(w t \%)$ & 12.86 & 23.78 & 0.00 & 0.00 & 0.00 & 0.00 & 0.00 & 0.00 & 99.88 & 0.11 & 0.00 & 0.00 & 0.00 & 0.00 \\
\hline Tol (wt\%) & 41.21 & 76.22 & 0.00 & 0.00 & 0.02 & 0.00 & 0.00 & 0.00 & 0.12 & 99.78 & 0.00 & 0.00 & 0.00 & 0.00 \\
\hline $\mathrm{ECH}(\mathrm{wt} \%)$ & 0.00 & 0.00 & 0.00 & 18.59 & 20.48 & 0.61 & 19.82 & 0.00 & 0.00 & 0.00 & 0.00 & 0.00 & 0.00 & 0.00 \\
\hline $\mathrm{EB}(\mathrm{wt} \%)$ & 3.20 & 0.00 & 6.97 & 6.30 & 6.89 & 0.02 & 6.72 & 0.00 & 0.00 & 0.00 & 0.00 & 0.00 & 0.00 & 0.00 \\
\hline $\mathrm{PX}(\mathrm{wt} \%)$ & 6.56 & 0.00 & 14.28 & 16.45 & 18.75 & 63.56 & 0.01 & 0.00 & 0.00 & 0.12 & 100.00 & 0.00 & 0.00 & 0.08 \\
\hline $\mathrm{MX}(\mathrm{wt} \%)$ & 13.88 & 0.00 & 30.22 & 35.17 & 39.18 & 0.02 & 38.24 & 0.09 & 0.00 & 0.00 & 0.00 & 0.56 & 0.00 & 0.00 \\
\hline $\mathrm{OX}(\mathrm{wt} \%)$ & 6.29 & 0.00 & 13.69 & 14.43 & 14.65 & 0.05 & 14.31 & 16.05 & 0.00 & 0.00 & 0.00 & 99.33 & 0.09 & 0.00 \\
\hline TMBen (wt\%) & 16.00 & 0.00 & 34.84 & 8.96 & 0.00 & 0.00 & 0.00 & 83.01 & 0.00 & 0.00 & 0.00 & 0.11 & 98.89 & 0.00 \\
\hline PDEB (wt\%) & 0.00 & 0.00 & 0.00 & 0.09 & 0.02 & 35.73 & 20.91 & 0.86 & 0.00 & 0.00 & 0.00 & 0.00 & 1.02 & 99.92 \\
\hline Stream & 15 & 16 & 17 & 18 & 19 & 20 & 21 & 22 & 23 & 24 & 25 & 26 & 27 & \\
\hline Flow $(\mathrm{t} / \mathrm{h})$ & 13.30 & 19.86 & 50.55 & 50.55 & 20.60 & 28.97 & 60.70 & 1.61 & 75.97 & 77.41 & 15.71 & 11.83 & 17.14 & \\
\hline Ben $(w t \%)$ & 0.00 & 0.00 & 0.00 & 0.00 & 23.78 & 0.00 & 0.00 & 0.00 & 0.10 & 0.08 & 0.08 & 0.00 & 0.00 & \\
\hline Tol (wt\%) & 0.00 & 0.00 & 0.00 & 0.00 & 76.22 & 0.05 & 0.00 & 0.92 & 99.81 & 77.70 & 99.92 & 0.00 & 0.09 & \\
\hline $\mathrm{ECH}(\mathrm{wt} \%)$ & 0.00 & 0.00 & 25.04 & 25.03 & 0.00 & 0.39 & 20.84 & 6.97 & 0.00 & 0.00 & 0.00 & 0.95 & 0.00 & \\
\hline $\mathrm{EB}(w \mathrm{t} \%)$ & 0.00 & 0.00 & 8.48 & 6.07 & 0.00 & 0.01 & 7.06 & 0.22 & 0.00 & 0.00 & 0.00 & 0.03 & 0.00 & \\
\hline $\mathrm{PX}(\mathrm{wt} \%)$ & 0.00 & 0.03 & 0.01 & 17.20 & 0.00 & 96.13 & 18.44 & 30.52 & 0.09 & 20.97 & 0.00 & 98.78 & 94.30 & \\
\hline MX (wt\%) & 0.00 & 0.00 & 48.30 & 36.89 & 0.00 & 1.68 & 39.42 & 30.08 & 0.00 & 0.62 & 0.00 & 0.04 & 2.80 & \\
\hline $\mathrm{OX}(\mathrm{wt} \%)$ & 0.08 & 0.05 & 18.05 & 14.68 & 0.00 & 1.69 & 14.23 & 30.35 & 0.00 & 0.62 & 0.00 & 0.07 & 2.80 & \\
\hline TMBen (wt $\%)$ & 0.00 & 0.00 & 0.00 & 0.00 & 0.00 & 0.00 & 0.00 & 0.00 & 0.00 & 0.00 & 0.00 & 0.00 & 0.00 & \\
\hline PDEB (wt\%) & 99.92 & 99.92 & 0.12 & 0.12 & 0.00 & 0.05 & 0.00 & 0.93 & 0.00 & 0.00 & 0.00 & 0.13 & 0.00 & \\
\hline
\end{tabular}


S13. Case G results

Table S55 Optimization of the modified aromatics complex with toluene methylation $(\mathrm{G})$

\begin{tabular}{|c|c|c|c|c|c|}
\hline & Step 1 & Step 2 & Step 3 & Step 4 & Step 5 \\
\hline Profit $\left(\$ / t_{\text {Reformate }}\right)$ & 1043.0 & 1043.6 & 1046.5 & 1046.6 & 1046.8 \\
\hline$\Delta$ Revenue $\times 10^{-3}(\$ / \text { year })^{\mathrm{a}}$ & 26750 & 26922 & 27783 & 27822 & 27884 \\
\hline \multicolumn{6}{|l|}{ Operation Cost $(\$ / \mathrm{h})$ : } \\
\hline Aromatics Extraction & 353.6 & 353.6 & 353.6 & 353.6 & 353.6 \\
\hline Benzene Column & 142.7 & 142.8 & 142.8 & 142.8 & 142.8 \\
\hline Xylene Splitter & 316.0 & 317.5 & 318.1 & 316.5 & 316.4 \\
\hline Extract Column & 342.8 & 329.2 & 281.3 & 282.4 & 279.7 \\
\hline Raffinate Column & 615.1 & 598.8 & 552.3 & 552.6 & 549.2 \\
\hline Isomerization & 399.5 & 401.6 & 402.6 & 399.7 & 399.6 \\
\hline$o$-Xylene Column & 62.6 & 62.8 & 62.8 & 62.7 & 62.7 \\
\hline Toluene Methylation & 1061.8 & 1068.4 & 1067.8 & 1067.8 & 1067.6 \\
\hline Toluene Column & 855.7 & 856.3 & 855.7 & 855.7 & 855.7 \\
\hline \multicolumn{6}{|l|}{ SMB: } \\
\hline Configuration & $5-9-7-3$ & $7-7-5-5$ & $7-7-5-5$ & $5-9-7-3$ & $6-6-8-4$ \\
\hline Switching time (s) & 66 & 66 & 106 & 112 & 112 \\
\hline Productivity $\left(\mathrm{kg} / \mathrm{hm}^{3}\right)$ & 122.75 & 122.72 & 122.65 & 122.68 & 122.67 \\
\hline Desorbent consumption $(\mathrm{kg} / \mathrm{kg})$ & 3.05 & 2.64 & 1.51 & 1.57 & 1.50 \\
\hline Recovery $(\%)$ & 99.43 & 99.27 & 99.19 & 99.44 & 99.45 \\
\hline Total $\Delta \mathrm{P}(\mathrm{kPa})$ & 230 & 229 & 126 & 120 & 124 \\
\hline Maximum $\Delta \mathrm{P} /$ bed $(\mathrm{kPa})$ & 10.9 & 11.0 & 6.5 & 6.2 & 6.2 \\
\hline \multicolumn{6}{|l|}{ Toluene Methylation: } \\
\hline Toluene conversion (\%) & 20.69 & 20.69 & 20.71 & 20.71 & 20.71 \\
\hline$p$-Xylene selectivity (\%) & 94.31 & 94.31 & 94.30 & 94.30 & 94.30 \\
\hline Toluene/MeOH (mol/mol) & 1.69 & 1.69 & 1.69 & 1.69 & 1.69 \\
\hline Water/Hydrocarbons (mol/mol) & 0.29 & 0.29 & 0.29 & 0.29 & 0.29 \\
\hline Space time $\left(\mathrm{g}_{\mathrm{cat}} \mathrm{h} / \mathrm{mol}_{\mathrm{Tol}}\right)$ & 3.57 & 3.57 & 3.58 & 3.58 & 3.58 \\
\hline $\mathrm{MeOH}$ loss $(\%)$ & 36.59 & 36.59 & 36.54 & 36.54 & 36.54 \\
\hline
\end{tabular}

a Compared to the base case (A0) assuming 330 days of operation per year. 
Table S56 Mass balance for the modified aromatics complex with toluene methylation after step 1 (G1)

\begin{tabular}{|c|c|c|c|c|c|c|c|c|c|c|c|c|}
\hline Stream & 1 & 2 & 3 & 4 & 5 & 6 & 7 & 8 & 9 & 10 & 11 & 12 \\
\hline Flow (t/h) & 38.10 & 20.60 & 17.50 & 68.66 & 78.44 & 59.22 & 102.73 & 7.35 & 4.89 & 59.82 & 27.37 & 1.19 \\
\hline Ben $(w t \%)$ & 12.86 & 23.78 & 0.00 & 0.00 & 0.00 & 0.00 & 0.00 & 0.00 & 99.88 & 0.11 & 0.00 & 0.00 \\
\hline Tol (wt\%) & 41.21 & 76.22 & 0.00 & 0.00 & 0.02 & 0.00 & 0.00 & 0.00 & 0.12 & 99.78 & 0.00 & 0.00 \\
\hline $\mathrm{ECH}(\mathrm{wt} \%)$ & 0.00 & 0.00 & 0.00 & 18.65 & 16.33 & 0.01 & 12.46 & 0.00 & 0.00 & 0.00 & 0.03 & 0.00 \\
\hline $\mathrm{EB}(\mathrm{wt} \%)$ & 3.20 & 0.00 & 6.97 & 6.30 & 5.51 & 0.01 & 4.21 & 0.00 & 0.00 & 0.00 & 0.01 & 0.00 \\
\hline $\mathrm{PX}(w \mathrm{t} \%)$ & 6.56 & 0.00 & 14.28 & 16.46 & 34.99 & 46.12 & 0.15 & 0.00 & 0.00 & 0.12 & 99.70 & 0.00 \\
\hline $\mathrm{MX}(\mathrm{wt} \%)$ & 13.88 & 0.00 & 30.22 & 35.19 & 31.41 & 0.01 & 23.98 & 0.09 & 0.00 & 0.00 & 0.03 & 0.56 \\
\hline $\mathrm{OX}(\mathrm{wt} \%)$ & 6.29 & 0.00 & 13.69 & 14.43 & 11.74 & 0.05 & 8.98 & 16.17 & 0.00 & 0.00 & 0.10 & 99.33 \\
\hline TMBen (wt\%) & 16.00 & 0.00 & 34.84 & 8.88 & 0.00 & 0.00 & 0.00 & 82.87 & 0.00 & 0.00 & 0.00 & 0.11 \\
\hline PDEB (wt\%) & 0.00 & 0.00 & 0.00 & 0.09 & 0.00 & 53.80 & 50.22 & 0.87 & 0.00 & 0.00 & 0.13 & 0.00 \\
\hline Stream & 13 & 14 & 15 & 16 & 17 & 18 & 19 & 20 & 21 & 22 & 23 & 24 \\
\hline Flow $(\mathrm{t} / \mathrm{h})$ & 6.16 & 31.85 & 51.56 & 83.42 & 51.16 & 51.16 & 20.60 & 15.71 & 75.53 & 76.96 & 17.14 & 61.31 \\
\hline Ben (wt\%) & 0.00 & 0.00 & 0.00 & 0.00 & 0.00 & 0.00 & 23.78 & 0.08 & 0.10 & 0.08 & 0.00 & 0.00 \\
\hline Tol (wt\%) & 0.00 & 0.00 & 0.00 & 0.00 & 0.00 & 0.00 & 76.22 & 99.92 & 99.81 & 77.58 & 0.09 & 0.00 \\
\hline $\mathrm{ECH}(\mathrm{wt} \%)$ & 0.00 & 0.00 & 0.00 & 0.00 & 25.02 & 25.03 & 0.00 & 0.00 & 0.00 & 0.00 & 0.00 & 20.89 \\
\hline $\mathrm{EB}(\mathrm{wt} \%)$ & 0.00 & 0.00 & 0.00 & 0.00 & 8.45 & 6.07 & 0.00 & 0.00 & 0.00 & 0.00 & 0.00 & 7.05 \\
\hline $\mathrm{PX}(\mathrm{wt} \%)$ & 0.00 & 0.08 & 0.00 & 0.03 & 0.31 & 17.20 & 0.00 & 0.00 & 0.09 & 21.07 & 94.21 & 18.43 \\
\hline $\mathrm{MX}(\mathrm{wt} \%)$ & 0.00 & 0.00 & 0.00 & 0.00 & 48.15 & 36.89 & 0.00 & 0.00 & 0.00 & 0.63 & 2.85 & 39.40 \\
\hline $\mathrm{OX}(\mathrm{wt} \%)$ & 0.09 & 0.00 & 0.08 & 0.05 & 17.95 & 14.68 & 0.00 & 0.00 & 0.00 & 0.63 & 2.85 & 14.22 \\
\hline TMBen (wt\%) & 98.88 & 0.00 & 0.00 & 0.00 & 0.00 & 0.00 & 0.00 & 0.00 & 0.00 & 0.00 & 0.00 & 0.00 \\
\hline PDEB (wt\%) & 1.03 & 99.92 & 99.92 & 99.92 & 0.12 & 0.12 & 0.00 & 0.00 & 0.00 & 0.00 & 0.00 & 0.00 \\
\hline
\end{tabular}


Table S57 Mass balance for the modified aromatics complex with toluene methylation after step 2 (G2)

\begin{tabular}{lcccccccccccc}
\hline Stream & 1 & 2 & 3 & 4 & 5 & 6 & 7 & 8 & 9 & 10 & 11 & 12 \\
\hline Flow (t/h) & 38.10 & 20.60 & 17.50 & 68.89 & 78.67 & 53.88 & 96.91 & 7.36 & 4.89 & 59.82 & 27.37 & 1.20 \\
Ben (wt\%) & 12.86 & 23.78 & 0.00 & 0.00 & 0.00 & 0.00 & 0.00 & 0.00 & 99.88 & 0.11 & 0.00 & 0.00 \\
Tol (wt\%) & 41.21 & 76.22 & 0.00 & 0.00 & 0.02 & 0.00 & 0.00 & 0.00 & 0.12 & 99.78 & 0.00 & 0.00 \\
ECH (wt\%) & 0.00 & 0.00 & 0.00 & 18.67 & 16.35 & 0.01 & 13.27 & 0.00 & 0.00 & 0.00 & 0.02 & 0.00 \\
EB (wt\%) & 3.20 & 0.00 & 6.97 & 6.30 & 5.52 & 0.01 & 4.47 & 0.00 & 0.00 & 0.00 & 0.02 & 0.00 \\
PX (wt\%) & 6.56 & 0.00 & 14.28 & 16.46 & 34.94 & 50.67 & 0.21 & 0.00 & 0.00 & 0.12 & 99.70 & 0.00 \\
MX (wt\%) & 13.88 & 0.00 & 30.22 & 35.19 & 31.43 & 0.01 & 25.51 & 0.09 & 0.00 & 0.00 & 0.03 & 0.55 \\
OX (wt\%) & 6.29 & 0.00 & 13.69 & 14.43 & 11.74 & 0.05 & 9.54 & 16.21 & 0.00 & 0.00 & 0.10 & 99.33 \\
TMBen (wt\%) & 16.00 & 0.00 & 34.84 & 8.85 & 0.00 & 0.00 & 0.00 & 82.82 & 0.00 & 0.00 & 0.00 & 0.11 \\
PDEB (wt\%) & 0.00 & 0.00 & 0.00 & 0.09 & 0.00 & 49.24 & 46.99 & 0.87 & 0.00 & 0.00 & 0.13 & 0.00 \\
\hline Stream & 13 & 14 & 15 & 16 & 17 & 18 & 19 & 20 & 21 & 22 & 23 & 24 \\
\hline Flow (t/h) & 6.16 & 26.52 & 45.51 & 72.03 & 51.40 & 51.40 & 20.60 & 15.71 & 75.53 & 76.96 & 17.14 & 61.54 \\
Ben (wt\%) & 0.00 & 0.00 & 0.00 & 0.00 & 0.00 & 0.00 & 23.78 & 0.08 & 0.10 & 0.08 & 0.00 & 0.00 \\
Tol (wt\%) & 0.00 & 0.00 & 0.00 & 0.00 & 0.00 & 0.00 & 76.22 & 99.92 & 99.81 & 77.58 & 0.09 & 0.00 \\
ECH (wt\%) & 0.00 & 0.00 & 0.00 & 0.00 & 25.02 & 25.03 & 0.00 & 0.00 & 0.00 & 0.00 & 0.00 & 20.91 \\
EB (wt\%) & 0.00 & 0.00 & 0.00 & 0.00 & 8.43 & 6.07 & 0.00 & 0.00 & 0.00 & 0.00 & 0.00 & 7.05 \\
PX (wt\%) & 0.00 & 0.08 & 0.00 & 0.03 & 0.39 & 17.20 & 0.00 & 0.00 & 0.09 & 21.07 & 94.21 & 18.43 \\
MX (wt\%) & 0.00 & 0.00 & 0.00 & 0.00 & 48.10 & 36.89 & 0.00 & 0.00 & 0.00 & 0.63 & 2.85 & 39.39 \\
OX (wt\%) & 0.09 & 0.00 & 0.08 & 0.05 & 17.93 & 14.68 & 0.00 & 0.00 & 0.00 & 0.63 & 2.85 & 14.22 \\
TMBen (wt\%) & 98.87 & 0.00 & 0.00 & 0.00 & 0.00 & 0.00 & 0.00 & 0.00 & 0.00 & 0.00 & 0.00 & 0.00 \\
PDEB (wt\%) & 1.04 & 99.92 & 99.92 & 99.92 & 0.12 & 0.12 & 0.00 & 0.00 & 0.00 & 0.00 & 0.00 & 0.00 \\
\hline
\end{tabular}


Table S58 Mass balance for the modified aromatics complex with toluene methylation after step 3 (G3)

\begin{tabular}{|c|c|c|c|c|c|c|c|c|c|c|c|c|}
\hline Stream & 1 & 2 & 3 & 4 & 5 & 6 & 7 & 8 & 9 & 10 & 11 & 12 \\
\hline Flow $(\mathrm{t} / \mathrm{h})$ & 38.10 & 20.60 & 17.50 & 69.02 & 78.80 & 38.42 & 81.72 & 7.36 & 4.89 & 59.78 & 27.36 & 1.20 \\
\hline Ben $(w t \%)$ & 12.86 & 23.78 & 0.00 & 0.00 & 0.00 & 0.00 & 0.00 & 0.00 & 99.88 & 0.11 & 0.00 & 0.00 \\
\hline Tol (wt\%) & 41.21 & 76.22 & 0.00 & 0.00 & 0.02 & 0.00 & 0.00 & 0.00 & 0.12 & 99.78 & 0.00 & 0.00 \\
\hline $\mathrm{ECH}(\mathrm{wt} \%)$ & 0.00 & 0.00 & 0.00 & 18.68 & 16.37 & 0.02 & 15.77 & 0.00 & 0.00 & 0.00 & 0.03 & 0.00 \\
\hline $\mathrm{EB}(\mathrm{wt} \%)$ & 3.20 & 0.00 & 6.97 & 6.30 & 5.52 & 0.04 & 5.30 & 0.00 & 0.00 & 0.00 & 0.06 & 0.00 \\
\hline $\mathrm{PX}(\mathrm{wt} \%)$ & 6.56 & 0.00 & 14.28 & 16.46 & 34.90 & 71.03 & 0.27 & 0.00 & 0.00 & 0.12 & 99.70 & 0.00 \\
\hline $\mathrm{MX}(\mathrm{wt} \%)$ & 13.88 & 0.00 & 30.22 & 35.20 & 31.44 & 0.01 & 30.32 & 0.09 & 0.00 & 0.00 & 0.01 & 0.55 \\
\hline $\mathrm{OX}(\mathrm{wt} \%)$ & 6.29 & 0.00 & 13.69 & 14.43 & 11.75 & 0.05 & 11.33 & 16.24 & 0.00 & 0.00 & 0.08 & 99.33 \\
\hline TMBen (wt\%) & 16.00 & 0.00 & 34.84 & 8.83 & 0.00 & 0.00 & 0.00 & 82.80 & 0.00 & 0.00 & 0.00 & 0.11 \\
\hline PDEB (wt\%) & 0.00 & 0.00 & 0.00 & 0.09 & 0.00 & 28.84 & 37.00 & 0.87 & 0.00 & 0.00 & 0.13 & 0.00 \\
\hline Stream & 13 & 14 & 15 & 16 & 17 & 18 & 19 & 20 & 21 & 22 & 23 & 24 \\
\hline Flow (t/h) & 6.16 & 11.05 & 30.20 & 41.25 & 51.53 & 51.53 & 20.60 & 15.71 & 75.48 & 76.91 & 17.13 & 61.66 \\
\hline & 0.00 & 0.00 & 0.00 & 0.00 & 0.00 & 0.00 & 23.78 & 0.08 & 0.10 & 0.08 & 0.00 & 0.00 \\
\hline Tol (wt\%) & 0.00 & 0.00 & 0.00 & 0.00 & 0.00 & 0.00 & 76.22 & 99.92 & 99.81 & 77.57 & 0.09 & 0.00 \\
\hline $\mathrm{ECH}(\mathrm{wt} \%)$ & 0.00 & 0.00 & 0.00 & 0.00 & 25.02 & 25.03 & 0.00 & 0.00 & 0.00 & 0.00 & 0.00 & 20.92 \\
\hline $\mathrm{EB}(\mathrm{wt} \%)$ & 0.00 & 0.00 & 0.00 & 0.00 & 8.41 & 6.07 & 0.00 & 0.00 & 0.00 & 0.00 & 0.00 & 7.05 \\
\hline $\mathrm{PX}(\mathrm{wt} \%)$ & 0.00 & 0.08 & 0.00 & 0.02 & 0.43 & 17.20 & 0.00 & 0.00 & 0.09 & 21.08 & 94.20 & 18.43 \\
\hline $\mathrm{MX}(\mathrm{wt} \%)$ & 0.00 & 0.00 & 0.00 & 0.00 & 48.09 & 36.89 & 0.00 & 0.00 & 0.00 & 0.64 & 2.86 & 39.39 \\
\hline OX (wt\%) & 0.09 & 0.00 & 0.08 & 0.06 & 17.93 & 14.68 & 0.00 & 0.00 & 0.00 & 0.64 & 2.86 & 14.22 \\
\hline TMBen (wt\%) & 98.87 & 0.00 & 0.00 & 0.00 & 0.00 & 0.00 & 0.00 & 0.00 & 0.00 & 0.00 & 0.00 & 0.00 \\
\hline PDEB (wt\%) & 1.04 & 99.92 & 99.92 & 99.92 & 0.12 & 0.12 & 0.00 & 0.00 & 0.00 & 0.00 & 0.00 & 0.00 \\
\hline
\end{tabular}


Table S59 Mass balance for the modified aromatics complex with toluene methylation after step 4 (G4)

\begin{tabular}{lcccccccccccc}
\hline Stream & 1 & 2 & 3 & 4 & 5 & 6 & 7 & 8 & 9 & 10 & 11 & 12 \\
\hline Flow (t/h) & 38.10 & 20.60 & 17.50 & 68.66 & 78.44 & 38.78 & 82.46 & 7.35 & 4.89 & 59.78 & 27.37 & 1.19 \\
Ben (wt\%) & 12.86 & 23.78 & 0.00 & 0.00 & 0.00 & 0.00 & 0.00 & 0.00 & 99.88 & 0.11 & 0.00 & 0.00 \\
Tol (wt\%) & 41.21 & 76.22 & 0.00 & 0.00 & 0.02 & 0.00 & 0.00 & 0.00 & 0.12 & 99.78 & 0.00 & 0.00 \\
ECH (wt\%) & 0.00 & 0.00 & 0.00 & 18.65 & 16.33 & 0.03 & 15.52 & 0.00 & 0.00 & 0.00 & 0.04 & 0.00 \\
EB (wt\%) & 3.20 & 0.00 & 6.97 & 6.30 & 5.51 & 0.03 & 5.23 & 0.00 & 0.00 & 0.00 & 0.04 & 0.00 \\
PX (wt\%) & 6.56 & 0.00 & 14.28 & 16.46 & 34.98 & 70.39 & 0.19 & 0.00 & 0.00 & 0.12 & 99.70 & 0.00 \\
MX (wt\%) & 13.88 & 0.00 & 30.22 & 35.19 & 31.42 & 0.02 & 29.88 & 0.09 & 0.00 & 0.00 & 0.02 & 0.56 \\
OX (wt\%) & 6.29 & 0.00 & 13.69 & 14.43 & 11.74 & 0.05 & 11.18 & 16.17 & 0.00 & 0.00 & 0.07 & 99.33 \\
TMBen (wt\%) & 16.00 & 0.00 & 34.84 & 8.88 & 0.00 & 0.00 & 0.00 & 82.87 & 0.00 & 0.00 & 0.00 & 0.11 \\
PDEB (wt\%) & 0.00 & 0.00 & 0.00 & 0.09 & 0.00 & 29.49 & 38.01 & 0.87 & 0.00 & 0.00 & 0.13 & 0.00 \\
\hline Stream & 13 & 14 & 15 & 16 & 17 & 18 & 19 & 20 & 21 & 22 & 23 & 24 \\
\hline Flow (t/h) & 6.16 & 11.41 & 31.30 & 42.71 & 51.16 & 51.16 & 20.60 & 15.71 & 75.48 & 76.91 & 17.13 & 61.30 \\
Ben (wt\%) & 0.00 & 0.00 & 0.00 & 0.00 & 0.00 & 0.00 & 23.78 & 0.08 & 0.10 & 0.08 & 0.00 & 0.00 \\
Tol (wt\%) & 0.00 & 0.00 & 0.00 & 0.00 & 0.00 & 0.00 & 76.22 & 99.92 & 99.81 & 77.57 & 0.09 & 0.00 \\
ECH (wt\%) & 0.00 & 0.00 & 0.00 & 0.00 & 25.01 & 25.03 & 0.00 & 0.00 & 0.00 & 0.00 & 0.00 & 20.89 \\
EB (wt\%) & 0.00 & 0.00 & 0.00 & 0.00 & 8.43 & 6.07 & 0.00 & 0.00 & 0.00 & 0.00 & 0.00 & 7.05 \\
PX (wt\%) & 0.00 & 0.08 & 0.00 & 0.02 & 0.30 & 17.20 & 0.00 & 0.00 & 0.09 & 21.08 & 94.20 & 18.43 \\
MX (wt\%) & 0.00 & 0.00 & 0.00 & 0.00 & 48.16 & 36.89 & 0.00 & 0.00 & 0.00 & 0.64 & 2.86 & 39.40 \\
OX (wt\%) & 0.09 & 0.00 & 0.08 & 0.06 & 17.96 & 14.68 & 0.00 & 0.00 & 0.00 & 0.64 & 2.86 & 14.22 \\
TMBen (wt\%) & 98.88 & 0.00 & 0.00 & 0.00 & 0.00 & 0.00 & 0.00 & 0.00 & 0.00 & 0.00 & 0.00 & 0.00 \\
PDEB (wt\%) & 1.03 & 99.92 & 99.92 & 99.92 & 0.12 & 0.12 & 0.00 & 0.00 & 0.00 & 0.00 & 0.00 & 0.00 \\
\hline
\end{tabular}


Table S60 Mass balance for the modified aromatics complex with toluene methylation after step 5 (G5)

\begin{tabular}{lcccccccccccc}
\hline Stream & 1 & 2 & 3 & 4 & 5 & 6 & 7 & 8 & 9 & 10 & 11 & 12 \\
\hline Flow (t/h) & 38.10 & 20.60 & 17.50 & 68.64 & 78.42 & 37.91 & 81.43 & 7.35 & 4.89 & 59.78 & 27.37 & 1.19 \\
Ben (wt\%) & 12.86 & 23.78 & 0.00 & 0.00 & 0.00 & 0.00 & 0.00 & 0.00 & 99.88 & 0.11 & 0.00 & 0.00 \\
Tol (wt\%) & 41.21 & 76.22 & 0.00 & 0.00 & 0.02 & 0.00 & 0.00 & 0.00 & 0.12 & 99.78 & 0.00 & 0.00 \\
ECH (wt\%) & 0.00 & 0.00 & 0.00 & 18.65 & 16.32 & 0.03 & 15.71 & 0.00 & 0.00 & 0.00 & 0.04 & 0.00 \\
EB (wt\%) & 3.20 & 0.00 & 6.97 & 6.30 & 5.51 & 0.03 & 5.30 & 0.00 & 0.00 & 0.00 & 0.05 & 0.00 \\
PX (wt\%) & 6.56 & 0.00 & 14.28 & 16.46 & 34.99 & 72.00 & 0.19 & 0.00 & 0.00 & 0.12 & 99.69 & 0.00 \\
MX (wt\%) & 13.88 & 0.00 & 30.22 & 35.19 & 31.42 & 0.02 & 30.25 & 0.09 & 0.00 & 0.00 & 0.02 & 0.56 \\
OX (wt\%) & 6.29 & 0.00 & 13.69 & 14.43 & 11.74 & 0.06 & 11.31 & 16.16 & 0.00 & 0.00 & 0.08 & 99.33 \\
TMBen (wt\%) & 16.00 & 0.00 & 34.84 & 8.88 & 0.00 & 0.00 & 0.00 & 82.88 & 0.00 & 0.00 & 0.00 & 0.11 \\
PDEB (wt\%) & 0.00 & 0.00 & 0.00 & 0.09 & 0.00 & 27.87 & 37.24 & 0.87 & 0.00 & 0.00 & 0.13 & 0.00 \\
\hline Stream & 13 & 14 & 15 & 16 & 17 & 18 & 19 & 20 & 21 & 22 & 23 & 24 \\
\hline Flow (t/h) & 6.16 & 10.54 & 30.29 & 40.83 & 51.14 & 51.14 & 20.60 & 15.71 & 75.48 & 76.91 & 17.13 & 61.28 \\
Ben (wt\%) & 0.00 & 0.00 & 0.00 & 0.00 & 0.00 & 0.00 & 23.78 & 0.08 & 0.10 & 0.08 & 0.00 & 0.00 \\
Tol (wt\%) & 0.00 & 0.00 & 0.00 & 0.00 & 0.00 & 0.00 & 76.22 & 99.92 & 99.81 & 77.57 & 0.09 & 0.00 \\
ECH (wt\%) & 0.00 & 0.00 & 0.00 & 0.00 & 25.01 & 25.03 & 0.00 & 0.00 & 0.00 & 0.00 & 0.00 & 20.89 \\
EB (wt\%) & 0.00 & 0.00 & 0.00 & 0.00 & 8.43 & 6.07 & 0.00 & 0.00 & 0.00 & 0.00 & 0.00 & 7.06 \\
PX (wt\%) & 0.00 & 0.08 & 0.00 & 0.02 & 0.30 & 17.20 & 0.00 & 0.00 & 0.09 & 21.08 & 94.20 & 18.43 \\
MX (wt\%) & 0.00 & 0.00 & 0.00 & 0.00 & 48.17 & 36.89 & 0.00 & 0.00 & 0.00 & 0.64 & 2.86 & 39.40 \\
OX (wt\%) & 0.09 & 0.00 & 0.08 & 0.06 & 17.97 & 14.68 & 0.00 & 0.00 & 0.00 & 0.64 & 2.86 & 14.22 \\
TMBen (wt\%) & 98.88 & 0.00 & 0.00 & 0.00 & 0.00 & 0.00 & 0.00 & 0.00 & 0.00 & 0.00 & 0.00 & 0.00 \\
PDEB (wt\%) & 1.03 & 99.92 & 99.92 & 99.92 & 0.12 & 0.12 & 0.00 & 0.00 & 0.00 & 0.00 & 0.00 & 0.00 \\
\hline
\end{tabular}




\section{S14. References}

(1) Silady, P. J. UOP Isomar Process. In Handbook of Petroleum Refining Processes, Third ed.; McGraw-Hill: 2003; pp 2.39-2.46.

(2) Starkey, D. R.; Andrews, J. L.; Luo, S. L.; Knob, K. J. PxMax with Crystallization. An Integrated Process for High Purity Paraxylene Production; ExxonMobil Chemical Technology Licensing LLC: 2009.

(3) Valverde, J. L. Alquilación de tolueno con metanol mediante catalizadores de zeolita ZSM-5 modificados. Universidad Complutense de Madrid, Madrid, 1991.

(4) Silva, M. S. P.; Mota, J. P. B.; Rodrigues, A. E. Adsorption Equilibrium and Kinetics of the Parex' Feed and Desorbent Streams from Batch Experiments. Chem. Eng. Technol. 2014, 37, (9), 1541-1551.

(5) Silva, M. S. P.; Rodrigues, A. E.; Mota, J. P. B. Modeling and simulation of an industrialscale parex process. AlChE J. 2015, 61, (4), 1345-1363. 